\title{
A PERTURBATION OF THE GEOMETRIC SPECTRAL SEQUENCE IN KHOVANOV HOMOLOGY
}

\author{
SUCHARIT SARKAR, COTTON SEED, AND ZOLTÁN SZABÓ
}

\begin{abstract}
We study the relationship between Bar-Natan's perturbation in Khovanov homology and Szabó's geometric spectral sequence, and construct a link invariant that generalizes both into a common theory. We study a few properties of the new invariant, and introduce a family of $s$-invariants from the new theory in the same spirit as Rasmussen's $s$-invariant.
\end{abstract}

\section{INTRODUCTION}

In [Kho00] Khovanov categorified the Jones polynomial to construct the first link homology theory, usually known as the Khovanov homology $K h(L)$ of links $L \subset S^{3}$. Several variants and improvements were then constructed - the reduced version [Kho03], Lee's perturbation [Lee05], Bar-Natan's tangle invariant [BN05] (yielding a Bar-Natan perturbation similar to Lee's), Khovanov's tangle invariant [Kho02], functoriality for link cobordisms in $\mathbb{R}^{3} \times I$ [Jac04, BN05, Kho06], KhovanovRozansky's $\mathfrak{s l}(n)$-homology and HOMFLYPT homology [KR08a, KR08b], Ozsváth-RasmussenSzabó's odd Khovanov homology [ORSz13], Seidel-Smith's symplectic Khovanov homology [SS06] equipped with localization spectral sequences [SS10] - to name a few. In [Ras10] Rasmussen used Lee's perturbation to construct a numerical invariant $s(K)$ leading to the first combinatorial proof of a theorem due to Kronheimer-Mrowka [KM93] on the four-ball genus of torus knots. Khovanov homology was also used by $\mathrm{Ng}$ to construct a bound on the Thurston-Bennequin number of Legendrian links [Ng05]. In [OSz05] Ozsváth-Szabó constructed a spectral sequence from the reduced version of the Khovanov homology of the mirror a link to the Heegaard Floer homology of its branched double cover. A similar spectral sequence was constructed by Bloom [Blo11], but abutting to the monopole Floer homology of the branched double cover. Motivated by these spectral sequences, Szabó constructed the geometric spectral sequence [Szab], a combinatorial construction that shares many formal properties with its holomorphic geometry and gauge theory counterparts. On a slightly different note, Kronheimer-Mrowka constructed a spectral sequence from Khovanov homology to the instanton knot Floer homology [KM11], which in turn established that Khovanov homology detects the unknot.

In our present paper, we concern ourselves with the interplay between the Bar-Natan perturbation in Khovanov homology, as introduced in [BN05] and studied further in [Nao06, Tur06], and the geometric spectral sequence constructed by Szabó in [Szab]. We present a brief survey of the

Date: January 14, 2016.

2010 Mathematics Subject Classification. 57M25.

SS was partially supported by NSF CAREER Grant DMS-1350037.

ZSz was partially supported by NSF Grants DMS-1006006 and DMS-1309152. 
existing constructions in Section 2. We produce our new endomorphism and prove that it is a differential in Section 3. We devote Section 4 to proving invariance and Section 5 to studying a few properties of the new invariant. Finally in Section 6 we introduce a family of $s$-invariants, each mimicking Rasmussen's $s$-invariant. We summarize our construction, state a few of its salient features, and discuss a bit on the motivation.

Construction. (Definition 3.5 and Proposition 3.4) We construct a $\left(\mathrm{gr}_{h}, \mathrm{gr}_{q}\right)$-bigraded chain complex $\mathcal{C}_{\text {tot }}$ over $\mathbb{F}_{2}[H, W]$, with $H$ and $W$ in bigradings $(0,-2)$ and $(-1,-2)$ and the differential in bigrading $(1,0)$. The chain group is freely generated (over $\mathbb{F}_{2}[H, W]$ ) by the Khovanov generators coming from some link diagram. Setting $W=0$ recovers the Bar-Natan theory, while setting $H=0$ recovers Szabó's geometric spectral sequence. For link diagrams equipped with a single basepoint, one can construct two reduced versions, the minus version $\mathcal{C}_{\text {tot }}^{-}$as a subcomplex and the plus version $\mathcal{C}_{\text {tot }}^{+}$as the corresponding quotient complex.

Properties. We list a few key properties of these new invariants.

- (Proposition 4.2 and Corollary 4.3) The chain homotopy type of $\mathcal{C}_{\text {tot }}$ over $\mathbb{F}_{2}[H, W]$ is a link invariant, while the chain homotopy type of the reduced versions (along with the maps to and from the unreduced theory) are invariants of links equipped with a single basepoint.

- (Proposition 5.4) For an $l$-component link, the homology of the localized version $\{H\}^{-1} \mathcal{C}_{\text {tot }}=$ $\mathcal{C}_{\text {tot }} \otimes_{\mathbb{F}_{2}[H, W]} \mathbb{F}_{2}\left[H, H^{-1}, W\right]$ is $2^{l}$ copies of $\mathbb{F}_{2}\left[H, H^{-1}, W\right]$, with the copies corresponding to the $2^{l}$ possible orientations of $L$.

- (Proposition 6.7) Half the absolute value of each of the new $s$-invariants from Definition 6.4 is a lower bound for the four-ball genus of knots.

Motivation. One of the main motivations behind this construction is the Seidel-Smith symplectic Khovanov homology [SS06], which is conjecturally isomorphic to Khovanov homology (the conjecture has been verified over characteristic $0[\mathrm{AS}]$, although it remains open over other characteristics, in particular, over characteristic 2). To expound a bit, after viewing the link $L$ as a plat closure of a $2 n$-braid, and letting $p: \mathbb{C} \rightarrow \mathbb{C}$ denote the degree- $2 n$ polynomial whose zeroes are the $2 n$ points of the braid, Seidel and Smith consider the complex variety

$$
C:=\left\{(u, v, z) \in \mathbb{C}^{3} \mid u^{2}+v^{2}=p(z)\right\}
$$

and define symplectic Khovanov homology to be the Lagrangian Floer homology in the $n^{\text {th }}$ symmetric product $\operatorname{Sym}^{n}(C)$ (or rather, in a certain open subset $\mathcal{Y}$ of the Hilbert scheme $\operatorname{Hilb}^{n}(C$ ) which is a smooth resolution of $\operatorname{Sym}^{n}(C)$, see [Man06] for more details), with each Lagrangian being a product of $n$ disjoint spheres in $C$; one of the Lagrangians is standard, coming from the $n$ plats at either end, while the other comes from applying the braid group action.

There is a $\mathbb{Z} / 2$-action induced by $(u, v) \stackrel{\tau}{\rightarrow}(u,-v)$, and its fixed points can be identified with the complex curve

$$
C^{\tau}:=\left\{(u, z) \in \mathbb{C}^{2} \mid u^{2}=p(z)\right\}
$$

which, along with the $\alpha$ and $\beta$ curves (which are the fixed points of the spheres on $C$ ), is easily seen to be a Heegaard diagram (in the sense of [OSz04]) for the double branched cover of $L$; and the fixed points of the Lagrangians can be identified with the Lagrangian tori from Heegaard Floer homology. The fixed points $\mathcal{Y}^{\tau}$ may be viewed as the complement of a certain divisor in $\operatorname{Sym}^{n}\left(C^{\tau}\right)$; 
therefore, there is a spectral sequence relating the Floer homology in $\operatorname{Sym}^{n}\left(C^{\tau}\right)$ and $\mathcal{Y}^{\tau}$, which is conjecturally trivial. The Seidel-Smith localization theorem [SS10] produces a chain complex over $H^{*}\left(B \mathbb{Z} / 2 ; \mathbb{F}_{2}\right)=\mathbb{F}_{2}\left[w_{1}\right]$ inducing a spectral sequence relating the symplectic Khovanov homology (which is conjecturally isomorphic to Khovanov homology) and Lagrangian Floer homology in $\mathcal{Y}^{\tau}$ (which is conjecturally isomorphic to Heegaard Floer homology, namely Lagrangian Floer homology in $\operatorname{Sym}^{n}\left(C^{\tau}\right)$ ); and the spectral sequence conjecturally equals the Ozsváth-Szabó spectral sequence and the Szabó geometric spectral sequence.

On the other hand, we may also consider the $S^{1}$-action $(u, v) \stackrel{\eta_{e^{i \theta}}}{\longrightarrow}(u \cos \theta-v \sin \theta, u \sin \theta+$ $v \cos \theta$ ), whose fixed points are the $2 n$ zeroes of $p$

$$
C^{\eta}:=\{z \in \mathbb{C} \mid p(z)=0\} .
$$

The fixed points $\mathcal{Y}^{\eta}$ is the subspace of $\operatorname{Sym}^{n}\left(C^{\eta}\right)$ consisting of distinct points. Furthermore, each Lagrangian is a product of $n$ zero-spheres in $C^{\eta}$; one corresponds to the matching of the $2 n$ points induced by the plats, while the other is the matching gotten by applying the braid group action. It is easily seen that the Lagrangians intersect in $2^{l}$ points, where $l$ is the number of link components of $L$, and therefore the Lagrangian Floer homology in the discrete space $\mathcal{Y}^{\eta}$ is $2^{l}$-dimensional. If there were a Seidel-Smith localization theorem for $S^{1}$-actions, it would have produced a chain complex over $H^{*}\left(B S^{1} ; \mathbb{Z}\right)=\mathbb{Z}\left[c_{1}\right]$ inducing a spectral sequence from the symplectic Khovanov homology (over $\mathbb{Z}$ ) to $\mathbb{Z}^{2^{l}}$, and it is conceivable that this spectral sequence would equal the Bar-Natan spectral sequence.

The two actions described above actually combine to induce an $O(2)$ action. Therefore, one might expect that the above two theories can be combined into a single theory: over $\mathbb{F}_{2}$, we should expect a chain complex over $\mathbb{F}_{2}\left[w_{1}, w_{2}\right] \cong H^{*}\left(B O(2) ; \mathbb{F}_{2}\right)$, so that the specialization $w_{2}=0$ produces the Szabó chain complex while the specialization $w_{1}=0$ produces the Bar-Natan chain complex. In this paper, we construct such a theory where $W$ plays the role of $w_{1}$ and $H$ plays the role of $w_{2}$. The gradings also work out: symplectic Khovanov homology carries a single grading, which is conjectured to equal $\operatorname{gr}_{h}-\operatorname{gr}_{q}$ on the Khovanov side; the universal Stiefel-Whitney classes $w_{1}$ and $w_{2}$ live in gradings 1 and 2, respectively, while the formal variables $W$ and $H$ live in $\left(\mathrm{gr}_{h}, \mathrm{gr}_{q}\right)$-bigradings $(-1,-2)$ and $(0,-2)$, respectively.

Acknowledgment. We thank Kristen Hendricks, Robert Lipshitz, and Ciprian Manolescu for several illuminating conversations about the Seidel-Smith construction, in particular for pointing out the $O(2)$-action, Alexander Shumakovitch for some invaluable help during computations, and the referees for pointing out some mistakes and for several other helpful comments.

\section{BACKGROUND}

Before we define our complex, let us first review the Khovanov chain complex, the Bar-Natan theory, and the Szabó geometric spectral sequence. All the three existing variants and our new fourth variant are defined in similar settings, particularly if we are working over $\mathbb{F}_{2}$, which we are. The relevant aspects are presented below in an enumerated list and a few definitions.

(X-1) $L \subset \mathbb{R}^{3}$ is a link represented by an $n$-crossing link diagram $D \subset \mathbb{R}^{2}=\mathbb{R}^{2} \times\{0\} \subset \mathbb{R}^{3}$; let $n_{+}$(respectively, $n_{-}$) denote the number of positive (respectively, negative) crossings in $D$. 


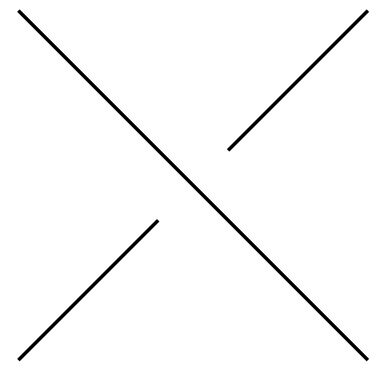

a: A crossing $c$.

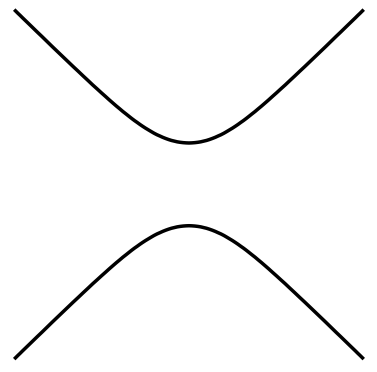

b: The 0-resolution at $c$.

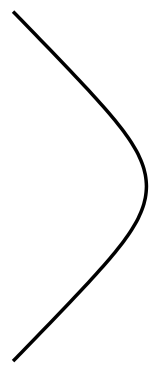

c: The 1-resolution at $c$.

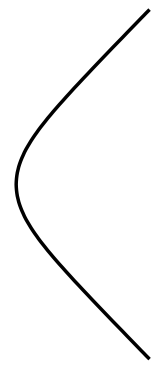

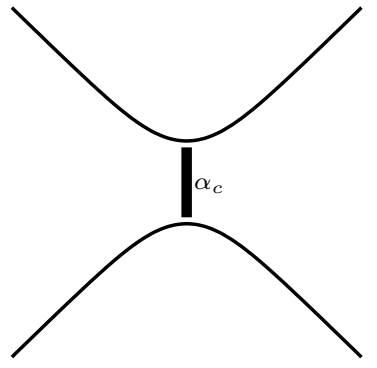

d: The 0-resolution at $c$ along with the surgery $\operatorname{arc} \alpha_{c}$.

Figure 2.1. A crossing, its 0-resolution, its 1-resolution, and its 0-resolution along with the surgery arc. If we forget the surgery arc, we get the 0-resolution; and if we perform embedded surgery along it, we get the 1-resolution.

Sometimes we work with a pointed link, that is, a link along with a single basepoint on it, and we represent it by a pointed link diagram $(D, p)$ where $p$ is some basepoint on $D$.

(X-2) Let $\mathfrak{C}$ be the set of crossings in $D$. For any subset $u \subseteq \mathfrak{C}$, let $D_{u}$ be the following complete resolution of $D$ : resolve a crossing $c$ by the 0-resolution if $c \notin u$, and resolve it by the 1-resolution otherwise, see Figure 2.1a-2.1c; let $Z\left(D_{u}\right)$ denote the set of circles in $D_{u}$.

(X-3) The Khovanov chain group $\mathcal{C}_{u}$ at $u$ is the exterior algebra generated by the circles in $D_{u}$; i.e., if $Z\left(D_{u}\right)=\left\{x_{1}, \ldots, x_{k}\right\}$, then $\mathcal{C}_{u}=\wedge^{*} \mathbb{F}_{2}\left\langle x_{1}, \ldots, x_{k}\right\rangle$; in particular, $\mathcal{C}_{u}$ has a distinguished basis $\mathfrak{B}_{u}$ consisting of the square-free monomials in $x_{i}$. If $(D, p)$ is a pointed link diagram, let $\mathfrak{B}_{u}^{-} \subset \mathfrak{B}_{u}$ be the subset consisting of the monomials where the circle containing $p$ appears; and let $\mathfrak{B}_{u}^{+}=\mathfrak{B}_{u} \backslash \mathfrak{B}_{u}^{-}$. The minus and the plus Khovanov chain groups, $\mathcal{C}_{u}^{-}$and $\mathcal{C}_{u}^{+}$, are the $\mathbb{F}_{2}$-vector subspaces of $\mathcal{C}_{u}$ generated by $\mathfrak{B}_{u}^{-}$and $\mathfrak{B}_{u}^{+}$, respectively.

(X-4) A Khovanov generator is a pair $(u, x)$ where $u \subseteq \mathfrak{C}$ and $x \in \mathfrak{B}_{u}$. The Khovanov generators are endowed with a bigrading $\left(\mathrm{gr}_{h}, \mathrm{gr}_{q}\right)$; the homological grading $\mathrm{gr}_{h}$ and a quantum grading $\operatorname{gr}_{q}$ are given by

$$
\begin{aligned}
& \operatorname{gr}_{h}((u, x))=-n_{-}+|u| \\
& \operatorname{gr}_{q}((u, x))=n_{+}-2 n_{-}+|u|+\left|Z\left(D_{u}\right)\right|-2 \operatorname{deg}(x) .
\end{aligned}
$$

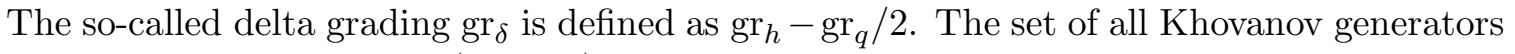
forms a basis for the total $\left(\mathrm{gr}_{h}, \mathrm{gr}_{q}\right.$ )-bigraded Khovanov chain group $\mathcal{C}=\underset{u \subseteq \mathfrak{C}}{\oplus} \mathcal{C}_{u}$.

(X-5) When working with a pointed link diagram, a minus (respectively, plus) Khovanov generator is a pair $(u, x)$ where $u \subseteq \mathfrak{C}$ and $x \in \mathfrak{B}_{u}^{-}$(respectively, $x \in \mathfrak{B}_{u}^{+}$). The set of all minus (respectively, plus) Khovanov generators forms a basis for the total minus (respectively, plus) Khovanov chain group $\mathcal{C}^{-}=\underset{u \subseteq \mathfrak{C}}{\oplus} \mathcal{C}_{u}^{-}$(respectively, $\mathcal{C}^{+}=\underset{u \subseteq \mathfrak{C}}{\oplus} \mathcal{C}_{u}^{+}$). The gr $q^{\text {-gradings }}$ are shifted by -1 (respectively, 1) for the minus (respectively, plus) theory; that is, $\mathcal{C}^{-}$ 
is a subspace of $\mathcal{C}\{-1\}$, while $\mathcal{C}^{+}$is a subspace of $\mathcal{C}\{1\}$ where \{\} denotes the grading shift operator for the second grading (with the usual convention $C\{a\}^{i, j} \cong C^{i, j+a}$ for any bigraded $\mathbb{F}_{2}$-vector space $C$, any bigrading $(i, j)$, and any shift $a$ for the second grading).

Definition 2.1. A resolution configuration $R$ consists of a set $Z(R)$ of smoothly embedded disjoint circles in $\mathbb{R}^{2}$ and a set $A(R)$ of properly embedded disjoint arcs in $\left(\mathbb{R}^{2}, Z(R)\right)$.

(1) The number of arcs in $A(R)$ is called the index of the resolution configuration.

(2) If the arcs in $A(R)$ are all oriented, then $R$ is called an oriented resolution configuration.

(3) Two (oriented) resolution configurations are equivalent if there is an isotopy, or equivalently, an orientation-preserving diffeomorphism of $S^{2}=\mathbb{R}^{2} \cup\{\infty\}$, that carries one to the other.

(4) The mirror $m(R)$ of a resolution configuration $R$ is obtained from $R$ by reflecting it along the line $\{0\} \times \mathbb{R}$.

(5) The reverse $r(R)$ of an oriented resolution configuration $R$ is obtained from $R$ by reversing the orientation of all the arcs in $A(R)$.

(6) The dual of an index- $k$ (oriented) resolution configuration $R$ is another index- $k$ (oriented) resolution configuration $R^{*}$, so that the circles in $Z\left(R^{*}\right)$ are obtained from the circles in $Z(R)$ by performing embedded surgeries along the arcs in $A(R)$, and the arcs in $A\left(R^{*}\right)$ are obtained by rotating the arcs in $A(R)$ by $90^{\circ}$ counter-clockwise.

(7) The circles in $Z(R)$ are called the starting circles of $R$, and the circles in $Z\left(R^{*}\right)$ are called the ending circles of $R$.

(8) The circles in $Z(R)$ that are disjoint from all the arcs in $A(R)$ are called the passive circles; the rest of the circles are called the active circles. Note, the passive circles of $R$ are in natural correspondence with the passive circles of $R^{*}$.

(9) A labeled resolution configuration $(R, x, y)$ is a resolution configuration $R$ along with a square-free monomial $x$ in the starting circles $\left\{x_{i}\right\}$ of $R$ and a square-free monomial $y$ in the ending circles $\left\{y_{i}\right\}$ of $R$. When we draw a labeled resolution configuration, we label the starting circles 0 or 1 : a starting circle appears in the monomial $x$ if it is labeled 1 , and does not appear in $x$ if it is labeled 0 ; and we draw the ending circles solid red or dashed blue: an ending circle appears in the monomial $y$ if it is solid and colored red, and does not appear in $y$ if it is dashed and colored blue.

(10) The dual $\left(R^{*}, y^{*}, x^{*}\right)$ of a labeled resolution configuration $(R, x, y)$ is defined as follows: a starting circle of $R^{*}$ appears in the monomial $y^{*}$ if and only if the corresponding ending circle of $R$ does not appear in $y$; and an ending circle of $R^{*}$ appears in the monomial $x^{*}$ if and only if the corresponding starting circle of $R$ does not appear in $x$. See Figure 2.2 for an oriented labeled resolution configuration and its dual.

Definition 2.2. For nested subsets $u \subseteq v \subseteq \mathfrak{C}$ with $|v|-|u|=k$, let $D_{u}^{v}$ denote the following index- $k$ resolution configuration: resolve a crossing $c$ by the 0 -resolution if $c \notin v$, resolve it by the 0 -resolution and add the surgery $\operatorname{arc} \alpha_{c}$ if $c \in v \backslash u$, and resolve it by the 1-resolution otherwise; see Figure 2.1. Note, the starting circles of $Z\left(D_{u}^{v}\right)$ are the circles in $Z\left(D_{u}\right)$ and the ending circles of $Z\left(D_{u}^{v}\right)$ are the circles of $Z\left(D_{v}\right)$.

A decoration is a choice of an orientation of all the arcs in $A\left(D_{\varnothing}^{\mathfrak{C}}\right)$. We specify a decoration by drawing an arrowhead near each crossing of the link diagram $D$ so that the $\operatorname{arcs}$ in $A\left(D_{\varnothing}^{\mathfrak{C}}\right)$ 


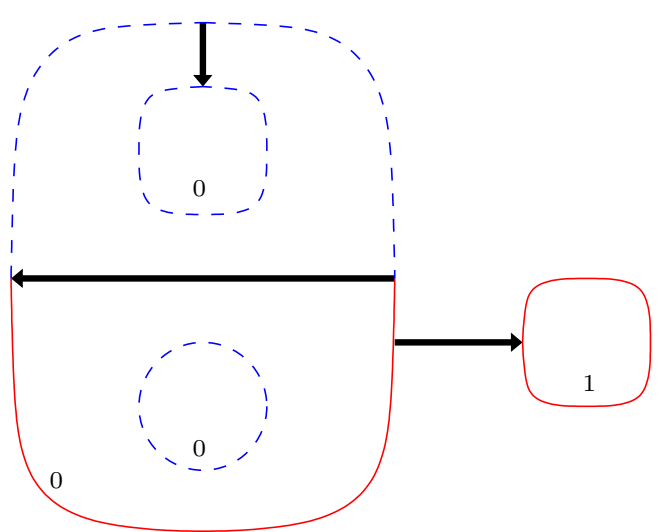

a: An oriented labeled resolution configuration $(R, x, y)$.

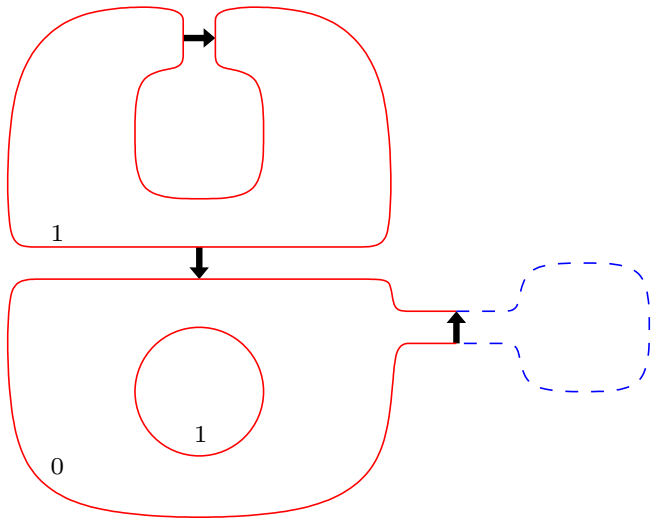

b: The dual oriented labeled resolution configuration $\left(R^{*}, y^{*}, x^{*}\right)$.

Figure 2.2. An oriented labeled resolution configuration and its dual. Recall, a starting circle appears in the monomial if it is labeled 1 and does not appear in the monomial if it is labeled 0 ; and an ending circle appears in the monomial if it is solid red and does not appear in the monomial if it is dashed blue.

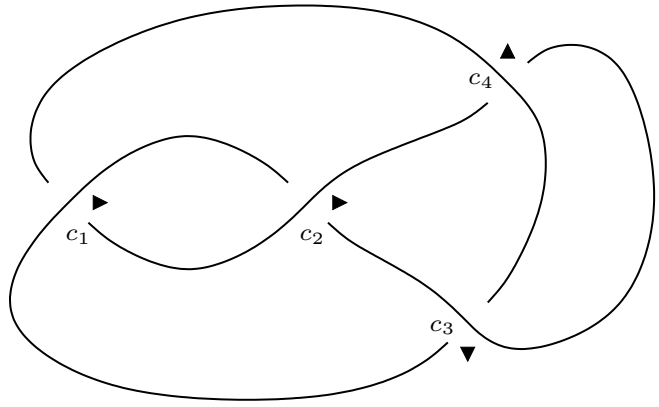

a: A decorated link diagram $D$ with $\mathfrak{C}=\left\{c_{1}, \ldots, c_{4}\right\}$.

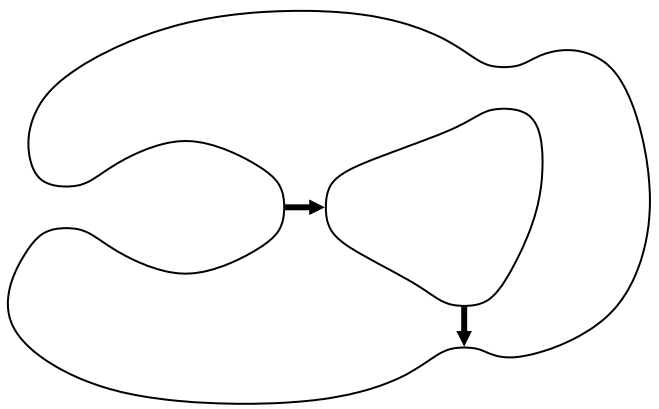

b: The oriented resolution configuration $D_{\left\{c_{1}\right\}}^{\left\{c_{1}, c_{2}, c_{3}\right\}}$.

FiguRE 2.3. A decorated link diagram for the figure eight knot, and one of the induced oriented resolution configurations.

are oriented in accord with the arrowheads. A decoration induces an orientation of all arcs in all resolution configurations $D_{u}^{v}$, see Figure 2.3.

Definition 2.3. A contribution function $\mathfrak{f}$ is a function from the set of all oriented labeled resolution configurations to $\mathbb{F}_{2}$. 
(1) $\mathfrak{f}$ satisfies the naturality rule if it is preserved under equivalence. That is, if there is an isotopy of $S^{2}$ carrying $(R, x, y)$ to $\left(R^{\prime}, x^{\prime}, y^{\prime}\right)$, then $\mathfrak{f}((R, x, y))=\mathfrak{f}\left(\left(R^{\prime}, x^{\prime}, y^{\prime}\right)\right)$.

(2) $\mathfrak{f}$ satisfies the conjugation rule if it is preserved under reversing. That is, for any $(R, x, y)$, $\mathfrak{f}((R, x, y))=\mathfrak{f}((r(R), x, y))$.

(3) $\mathfrak{f}$ satisfies the disoriented rule if it is preserved under an arbitrary re-orientation of arcs. That is, if $R$ and $R^{\prime}$ differ only in the orientation of their arcs, then $\mathfrak{f}((R, x, y))=\mathfrak{f}\left(\left(R^{\prime}, x, y\right)\right)$.

(4) $\mathfrak{f}$ satisfies the duality rule if it is preserved under dualizing and taking mirrors. That is, if $m\left(R^{*}, y^{*}, x^{*}\right)$ denotes the mirror of the dual of $(R, x, y)$, then $\mathfrak{f}\left(m\left(R^{*}, y^{*}, x^{*}\right)\right)=\mathfrak{f}((R, x, y))$.

(5) $\mathfrak{f}$ satisfies the extension rule if it only depends on the active part of the resolution configuration. That is, given $(R, x, y)$, let $R_{a}$ (respectively, $\left.x_{a}, y_{a}\right)$ be the active part of $R$ (respectively, $x, y$ ) and let $R_{p}$ (respectively, $x_{p}, y_{p}$ ) be the passive part of $R$ (respectively, $x, y)$ so that $R=R_{a} \coprod R_{p}$ (respectively, $x=x_{a} x_{p}, y=y_{a} y_{p}$ ); then

$$
\mathfrak{f}((R, x, y))= \begin{cases}\mathfrak{f}\left(\left(R_{a}, x_{a}, y_{a}\right)\right) & \text { if } x_{p}=y_{p}, \\ 0 & \text { otherwise. }\end{cases}
$$

(6) $\mathfrak{f}$ satisfies the filtration rule if it does not contribute whenever there is some point $p$ such that the starting circle containing $p$ is in the starting monomial, but the ending circle containing $p$ is not in the ending monomial. That is, if $\mathfrak{f}((R, x, y)) \neq 0$, then, every starting circle that appears in $x$ is disjoint from every ending circle that does not appear in $y$.

A contribution function $\mathfrak{f}$ satisfying the naturality rule defines an endomorphism $\mathbf{f}$ of the Khovanov chain group $\mathcal{C}$ coming from a decorated link diagram as follows. Given Khovanov generators $(u, x)$ and $(v, y)$,

$$
\langle\mathbf{f}((u, x)),(v, y)\rangle= \begin{cases}\mathfrak{f}\left(\left(D_{u}^{v}, x, y\right)\right) & \text { if } u \subseteq v, \\ 0 & \text { otherwise. }\end{cases}
$$

Clearly, if the contribution function $\mathfrak{f}$ satisfies the disoriented rule, then the endomorphism $\mathbf{f}$ does not depend on the choice of decoration of the link diagram. Also, if $\mathfrak{f}$ satisfies the filtration rule, and we are working with a pointed link diagram, then $\mathbf{f}$ restricts to an endomorphism of $\mathcal{C}^{-}$.

With these concepts in place, we are ready to recall the definitions of the Khovanov differential, the Bar-Natan differential, and the Szabó differential, although not entirely in their original forms.

Definition 2.4 ([Kho00, Section 4.2]). The Khovanov contribution function $\mathfrak{k}$ satisfies the naturality rule, the disoriented rule (and hence the conjugation rule), the duality rule, the extension rule, and the filtration rule. A labeled resolution configuration $(R, x, y)$ has non-zero contribution in $\mathfrak{k}$ if and only if $x$ and $y$ agree on the passive circles, and the active part is equivalent to one of the four configurations in Figure 2.4.

In the Merge configurations, there are two starting circles with a single arc between them. In the Merge-A configuration, both the starting and the ending monomials are 1, while in the Merge-B configuration, the starting monomial and the ending monomial each contains exactly one circle. The Split-A configuration and the Split-B configuration are the duals of the Merge-A configuration and the Merge-B configuration, respectively. 


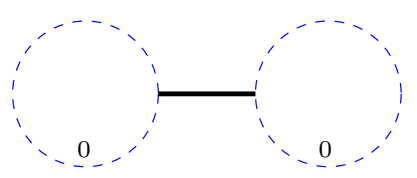

Merge-A

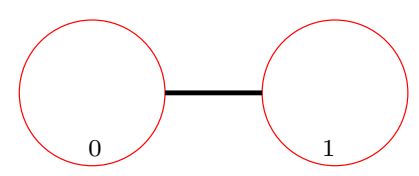

Merge-B

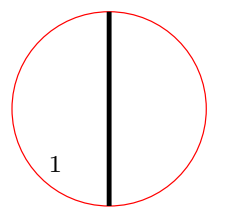

Split-A

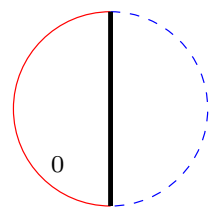

Split-B

Figure 2.4. The labeled configurations that contribute to the function $\mathfrak{k}$.

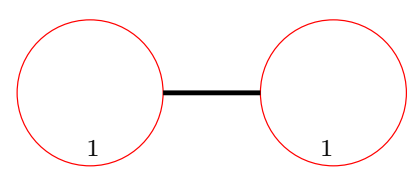

Merge-C

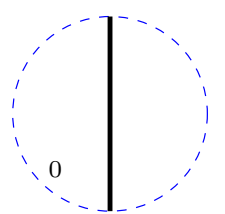

Split-C

FiguRE 2.5. The labeled configurations that contribute to the function $\mathfrak{b}$.

Starting with a link diagram, the Khovanov differential $\delta_{K h}=\mathbf{d}_{1}$ is the $(1,0)$-graded endomorphism on $\mathcal{C}$ induced by $\mathfrak{k}$,

$$
\left\langle\mathbf{d}_{1}((u, x)),(v, y)\right\rangle= \begin{cases}\mathfrak{k}\left(\left(D_{u}^{v}, x, y\right)\right) & \text { if } u \subseteq v, \\ 0 & \text { otherwise. }\end{cases}
$$

It is indeed a differential, i.e., $\left(\delta_{K h}\right)^{2}=0$, and the Khovanov chain complex is defined as

$$
\mathcal{C}_{K h}=\left(\mathcal{C}, \delta_{K h}\right)
$$

Since $\mathfrak{k}$ satisfies the filtration rule, for a pointed link diagram, $\delta_{K h}$ restricts to an endomorphism on $\mathcal{C}^{-}$. Therefore, we get a subcomplex $\mathcal{C}_{K h}^{-}$generated by $\mathcal{C}^{-}$and a quotient complex $\mathcal{C}_{K h}^{+}$generated by $\mathcal{C}^{+}$, which fit into a short exact sequence

$$
0 \rightarrow \mathcal{C}_{K h}^{-}\{1\} \rightarrow \mathcal{C}_{K h} \rightarrow \mathcal{C}_{K h}^{+}\{-1\} \rightarrow 0
$$

Definition 2.5 ([BN05, Section 9.3]). The Bar-Natan contribution function $\mathfrak{b}$ satisfies the naturality rule, the disoriented rule (and hence the conjugation rule), the duality rule, the extension rule, and the filtration rule. A labeled resolution configuration $(R, x, y)$ has non-zero contribution in $\mathfrak{b}$ if and only if $x$ and $y$ agree on the passive circles, and the active part is equivalent to one of the two configurations in Figure 2.5.

In the Merge-C configuration, there are two starting circles with a single arc between them, and both the starting and the ending monomials contain all the circles; the Split-C configuration is the dual of the Merge- $\mathrm{C}$ configuration. 
Starting with a link diagram, the Bar-Natan perturbation $\mathbf{h}_{1}$ is the (1,2)-graded endomorphism on $\mathcal{C}$ induced by $\mathfrak{b}$,

$$
\left\langle\mathbf{h}_{1}((u, x)),(v, y)\right\rangle= \begin{cases}\mathfrak{b}\left(\left(D_{u}^{v}, x, y\right)\right) & \text { if } u \subseteq v, \\ 0 & \text { otherwise. }\end{cases}
$$

The Bar-Natan differential is the (1,0)-graded endomorphism $\delta_{B N}=\mathbf{d}_{1}+H \mathbf{h}_{1}$ on $\mathcal{C} \otimes \mathbb{F}_{2}[H]$, with $H$ being a formal variable in $\left(\mathrm{gr}_{h}, \mathrm{gr}_{q}\right)$-bigrading $(0,-2)$. Once again, this is a differential, i.e., $\left(\delta_{B N}\right)^{2}=0$, and the Bar-Natan chain complex is defined as

$$
\mathcal{C}_{B N}=\left(\mathcal{C} \otimes \mathbb{F}_{2}[H], \delta_{B N}\right) .
$$

Clearly, $\mathcal{C}_{K h}$ is obtained from $\mathcal{C}_{B N}$ by setting $H=0$. The filtered and the localized versions are defined as

$$
\begin{aligned}
\mathcal{C}_{f B N} & =\mathcal{C}_{B N} /\{H=1\}=\left(\mathcal{C}, \mathbf{d}_{1}+\mathbf{h}_{1}\right) \\
\{H\}^{-1} \mathcal{C}_{B N} & =\mathcal{C}_{B N} \otimes_{\mathbb{F}_{2}[H]} \mathbb{F}_{2}\left[H, H^{-1}\right]=\left(\mathcal{C} \otimes \mathbb{F}_{2}\left[H, H^{-1}\right], \delta_{B N}\right) .
\end{aligned}
$$

Once again, since both $\mathfrak{k}$ and $\mathfrak{b}$ satisfy the filtration rule, for a pointed link diagram, we get a subcomplex $\mathcal{C}_{B N}^{-}$generated by $\mathcal{C}^{-}$and a quotient complex $\mathcal{C}_{B N}^{+}$generated by $\mathcal{C}^{+}$fitting into a short exact sequence over $\mathbb{F}_{2}[H]$

$$
0 \rightarrow \mathcal{C}_{B N}^{-}\{1\} \rightarrow \mathcal{C}_{B N} \rightarrow \mathcal{C}_{B N}^{+}\{-1\} \rightarrow 0
$$

Definition 2.6 ([Szab, Section 3-4]). The Szabó contribution function $\mathfrak{d}$ satisfies the naturality rule, the conjugation rule (but not the disoriented rule), the duality rule, the extension rule, and the filtration rule. An oriented labeled resolution configuration $(R, x, y)$ has non-zero contribution in $\mathfrak{d}$ if and only if $x$ and $y$ agree on the passive circles, and the active part is equivalent to a configuration from one of the five families in [Szab, Figure 3] or Figure 2.6.

In a Type-A configuration (Figure 2.6a), there are two starting circles, with some (at least one) parallel arcs between them; both the starting and the ending monomials are 1. A Type$\mathrm{B}$ configuration (Figure 2.6b) is the dual of a Type-A configuration. In a Type-C configuration (Figure 2.6c), there is a single starting circle, with some (at least one) parallel arcs inside, and some (at least one) parallel arcs outside, so that the inside arcs and the outside arcs are linked, and the orientations are as shown; both the starting and ending monomials are 1. A Type-D configuration (Figure 2.6d) is the mirror of the dual of a Type-C configuration. The Type-E configurations are slightly harder to describe. In the Type-E configuration from Figure 2.6e, there is exactly one circle, call it the special starting circle, that does not appear in the starting monomial; and there is exactly one circle, call it the special ending circle, that does appear in the ending monomial. All the non-special starting circles are incident to exactly one arc; and all the non-special ending circles are incident to exactly one dual arc. The arcs either run from the non-special starting circles to the special starting circle, or run from the special starting circle to itself; in the latter case, they are oriented as shown (depending on whether they are inside or outside of the special starting circle). We also impose the condition that the index of the configuration is at least one. The Type-E configuration from Figure 2.6f is the reverse of the Type-E configuration from Figure 2.6e. 


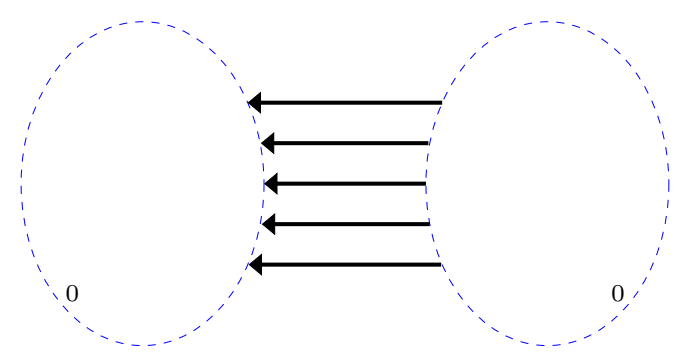

a: Type-A configuration.

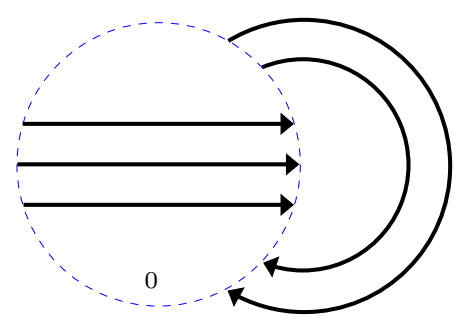

c: Type-C configuration.

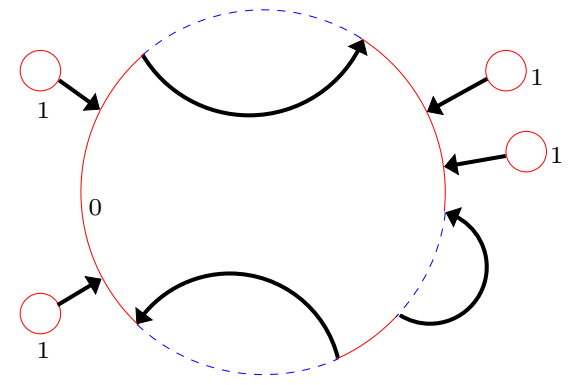

e: Type-E configuration.

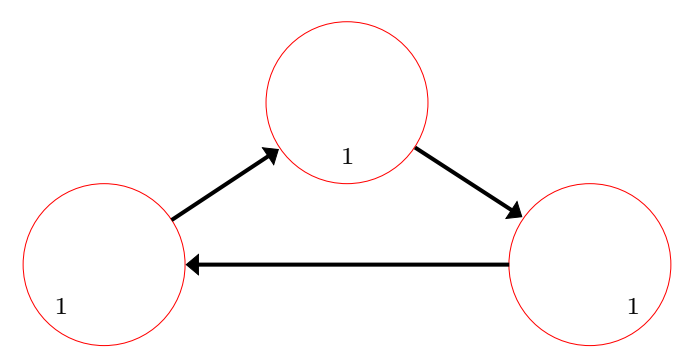

b: Type-B configuration.

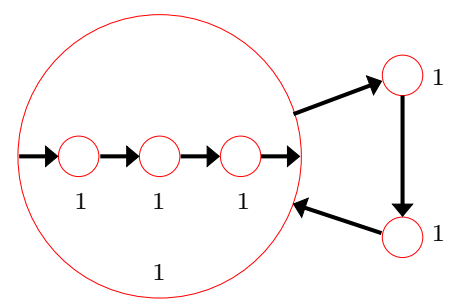

d: Type-D configuration.

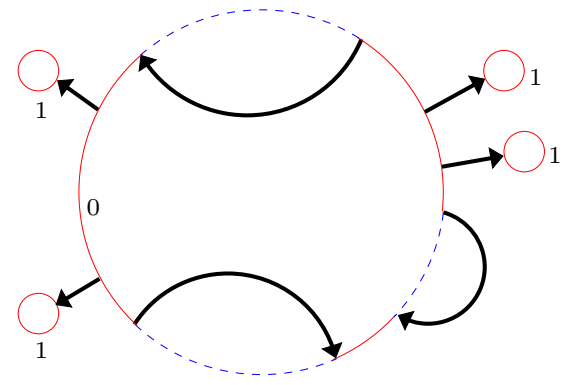

f: Type-E configuration.

FiguRE 2.6. The oriented labeled configurations that contribute to the function $\mathfrak{d}$.

Working with a decorated link diagram, the induced endomorphism $\mathbf{d}$ of $\mathcal{C}$, defined as

$$
\langle\mathbf{d}((u, x)),(v, y)\rangle= \begin{cases}\mathfrak{d}\left(\left(D_{u}^{v}, x, y\right)\right) & \text { if } u \subseteq v, \\ 0 & \text { otherwise }\end{cases}
$$

increases the homological grading gr $\mathrm{gr}_{h}$ by at least one, and increases delta grading $\operatorname{gr}_{\delta}=\operatorname{gr}_{h}-\operatorname{gr}_{q} / 2$ by exactly one; therefore, $\mathbf{d}$ can be (uniquely) written as $\sum_{i=1}^{\infty} \mathbf{d}_{i}$, where $\mathbf{d}_{i}$ increases $\mathrm{gr}_{h}$ by exactly $i$ and increases $\operatorname{gr}_{q}$ by exactly $2 i-2$. Indeed, $\mathbf{d}_{1}$ is independent of the initial decoration, and is the Khovanov differential from Definition 2.4. The Szabó geometric differential is the (1,0)-graded endomorphism $\delta_{S z}=\mathbf{d}_{1}+W \mathbf{d}_{2}+W^{2} \mathbf{d}_{3}+\cdots$ on $\mathcal{C} \otimes \mathbb{F}_{2}[W]$ with $W$ being a formal variable in $\left(\mathrm{gr}_{h}, \mathrm{gr}_{q}\right)$-bigrading $(-1,-2)$. It is shown in [Szab, Section 6] that this is a differential, i.e., 
$\left(\delta_{S z}\right)^{2}=0$, and the Szabó chain complex is defined as

$$
\mathcal{C}_{S z}=\left(\mathcal{C} \otimes \mathbb{F}_{2}[W], \delta_{S z}\right) .
$$

The Khovanov chain complex $\mathcal{C}_{K h}$ can be recovered from $\mathcal{C}_{S z}$ by setting $W=0$. The filtered version is defined as

$$
\mathcal{C}_{f S z}=\mathcal{C}_{S z} /\{W=1\}=\left(\mathcal{C}, \mathbf{d}_{1}+\mathbf{d}_{2}+\mathbf{d}_{3}+\ldots\right)=(\mathcal{C}, \mathbf{d}) .
$$

As before, since $\mathfrak{d}$ satisfies the filtration rule, for a pointed decorated link diagram, we get a subcomplex $\mathcal{C}_{S z}^{-}$generated by $\mathcal{C}^{-}$and a quotient complex $\mathcal{C}_{S z}^{+}$generated by $\mathcal{C}^{+}$fitting into a short exact sequence over $\mathbb{F}_{2}[W]$

$$
0 \rightarrow \mathcal{C}_{S z}^{-}\{1\} \rightarrow \mathcal{C}_{S z} \rightarrow \mathcal{C}_{S z}^{+}\{-1\} \rightarrow 0
$$

Therefore, to summarize the existing story, we have a $(1,2)$-graded endomorphism $\mathbf{h}_{1}$, and for all $i>0,(i, 2 i-2)$-graded endomorphisms $\mathbf{d}_{i}$ for $\mathcal{C}$, satisfying $\left(\mathbf{d}_{1}+\mathbf{h}_{1}\right)^{2}=\mathbf{d}^{2}=0$, where $\mathbf{d}=\left(\mathbf{d}_{1}+\mathbf{d}_{2}+\mathbf{d}_{3}+\cdots\right)$. From grading considerations, this breaks up into the following equations

$$
\begin{aligned}
\mathbf{h}_{1}^{2}=0 \quad\left[\mathbf{h}_{1}: \mathbf{d}_{1}\right]=0 & \mathbf{d}_{1}^{2}=0 \\
{\left[\mathbf{d}_{1}: \mathbf{d}_{2}\right] } & =0 \\
{\left[\mathbf{d}_{1}: \mathbf{d}_{3}\right]+\mathbf{d}_{2}^{2} } & =0 \\
\ldots &
\end{aligned}
$$

Therefore, the Khovanov chain complex, the Bar-Natan chain complex, and the Szabó geometric chain complex can be defined as the following objects, respectively:

$$
\begin{aligned}
\mathcal{C}_{K h} & =\left(\mathcal{C}, \delta_{K h}:=\mathbf{d}_{1}\right) \\
\mathcal{C}_{B N} & =\left(\mathcal{C} \otimes \mathbb{F}_{2}[H], \delta_{B N}:=\mathbf{d}_{1}+H \mathbf{h}_{1}\right) \\
\mathcal{C}_{S z} & =\left(\mathcal{C} \otimes \mathbb{F}_{2}[W], \delta_{S z}:=\mathbf{d}_{1}+W \mathbf{d}_{2}+W^{2} \mathbf{d}_{3}+\cdots\right)
\end{aligned}
$$

Here $H$ and $W$ are formal variables carrying $\left(\mathrm{gr}_{h}, \mathrm{gr}_{q}\right)$-bigradings $(0,-2)$ and $(-1,-2)$, respectively, so that above complexes are also bigraded with the differential being of grading $(1,0)$.

In the next section, we will extend the definition of $\mathbf{h}_{1}$ to construct $(i, 2 i)$-graded endomorphisms $\mathbf{h}_{i}$ for all $i$. Setting $\mathbf{h}=\left(\mathbf{h}_{1}+\mathbf{h}_{2}+\mathbf{h}_{3}+\cdots\right)$, it will satisfy $\mathbf{h}^{2}=[\mathbf{h}: \mathbf{d}]=0$. Expanding along gradings, we will get equations

$$
\begin{aligned}
\mathbf{h}_{1}^{2}=0 & {\left[\mathbf{h}_{1}: \mathbf{d}_{1}\right]=0 } \\
{\left[\mathbf{h}_{1}: \mathbf{h}_{1}\right]=0 } & \left.=\mathbf{h}_{1}: \mathbf{d}_{2}\right]+\left[\mathbf{h}_{2}: \mathbf{d}_{1}\right]=0 \\
{\left[\mathbf{h}_{1}: \mathbf{h}_{3}\right]+\mathbf{h}_{2}^{2}=0 } & {\left[\mathbf{h}_{1}: \mathbf{d}_{3}\right]+\left[\mathbf{h}_{2}: \mathbf{d}_{2}\right]+\left[\mathbf{h}_{3}: \mathbf{d}_{1}\right]=0 }
\end{aligned}
$$

and we will define our chain complex as

$$
\mathcal{C}_{\text {tot }}=\left(\mathcal{C} \otimes \mathbb{F}_{2}[H, W], \delta_{\text {tot }}:=\mathbf{d}_{1}+W \mathbf{d}_{2}+W^{2} \mathbf{d}_{3}+\cdots+H \mathbf{h}_{1}+H W \mathbf{h}_{2}+H W^{2} \mathbf{h}_{3}+\cdots\right) .
$$

with the differential lying in grading $(1,0)$. Clearly, $\mathcal{C}_{B N}$ and $\mathcal{C}_{S z}$ can be obtained from $\mathcal{C}_{\text {tot }}$ by setting $W=0$ and $H=0$, respectively, while $\mathcal{C}_{K h}$ can be obtained by setting both to zero; therefore, $\mathcal{C}_{\text {tot }}$ will be the master chain complex. 

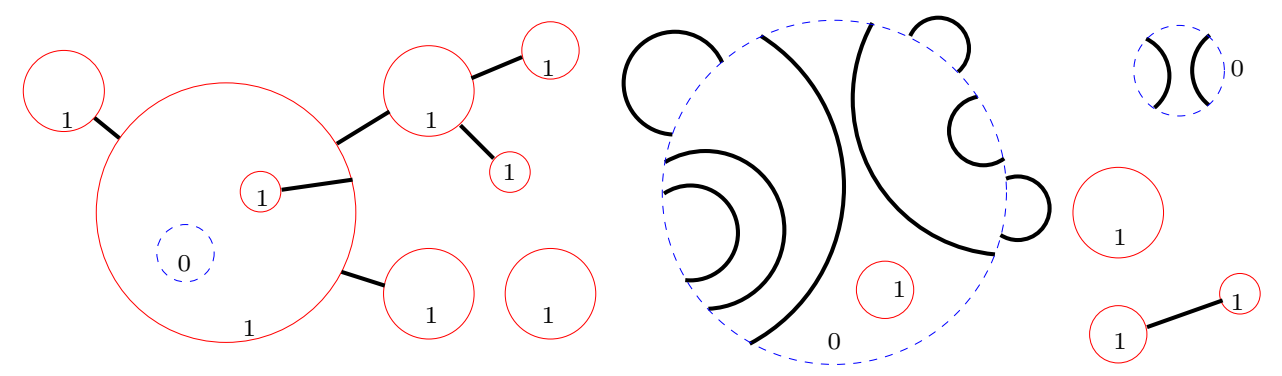

Figure 3.1. A labeled configuration that contributes to $\mathfrak{h}$.

The endomorphism $\mathbf{d}$ was defined from a contribution function $\mathfrak{d}$, and $\mathbf{d}_{i}$ was simply the graded part of $\mathbf{d}$ that increased the homological grading by $i$. We will also define the endomorphism $\mathbf{h}$ from some contribution function $\mathfrak{h}$, and $\mathbf{h}_{i}$ will be graded part of $\mathbf{h}$ that increases the homological grading by $i$. Therefore, in order to introduce our chain complex, all that remains is to define the contribution function $\mathfrak{h}$. We turn to this in the next section.

\section{Construction}

Definition 3.1. A tree is a labeled resolution configuration of some index $k \geq 0$, with exactly $(k+1)$ starting circles, exactly one ending circle, and no passive circles, and with all the starting circles appearing in the starting monomial, and the ending circle appearing in the ending monomial.

Definition 3.2. The contribution function $\mathfrak{h}$ is defined as follows: It is non-zero for a labeled resolution configuration $(R, x, y)$ if only if $(R, x, y)$ is a disjoint union of trees and dual trees, and the index of $R$ is at least one. See Figure 3.1 for a resolution configuration that contributes to $\mathfrak{h}$.

Lemma 3.3. The contribution function $\mathfrak{h}$ satisfies the naturality rule, the disoriented rule (and hence the conjugation rule), the duality rule, the extension rule, and the filtration rule. The induced endomorphism $\mathbf{h}$ of $\mathcal{C}$ coming from some link diagram $D$ (or of $\mathcal{C}^{ \pm}$coming from some pointed link diagram $(D, p))$, defined as

$$
\langle\mathbf{h}((u, x)),(v, y)\rangle= \begin{cases}\mathfrak{h}\left(\left(D_{u}^{v}, x, y\right)\right) & \text { if } u \subseteq v, \\ 0 & \text { otherwise },\end{cases}
$$

increases the homological grading $\mathrm{gr}_{h}$ by at least one, and preserves the delta grading $\operatorname{gr}_{\delta}=\operatorname{gr}_{h}-$ $\operatorname{gr}_{q} / 2$; therefore, $\mathbf{h}$ can be (uniquely) written as $\sum_{i=1}^{\infty} \mathbf{h}_{i}$, where $\mathbf{h}_{i}$ increases $\operatorname{gr}_{h}$ by $i$ and increases $\operatorname{gr}_{q}$ by $2 i$. Furthermore, $\mathbf{h}_{1}$ is the Bar-Natan perturbation from Definition 2.5.

Proof. This is immediate from the definitions.

Proposition 3.4. The endomorphism $\mathbf{d}+\mathbf{h}$ is a differential on $\mathcal{C}$.

The main aim of this section is to prove the above statement, and we will give the proof at the very end. In the meantime, Proposition 3.4 allows us to define the following chain complex. 


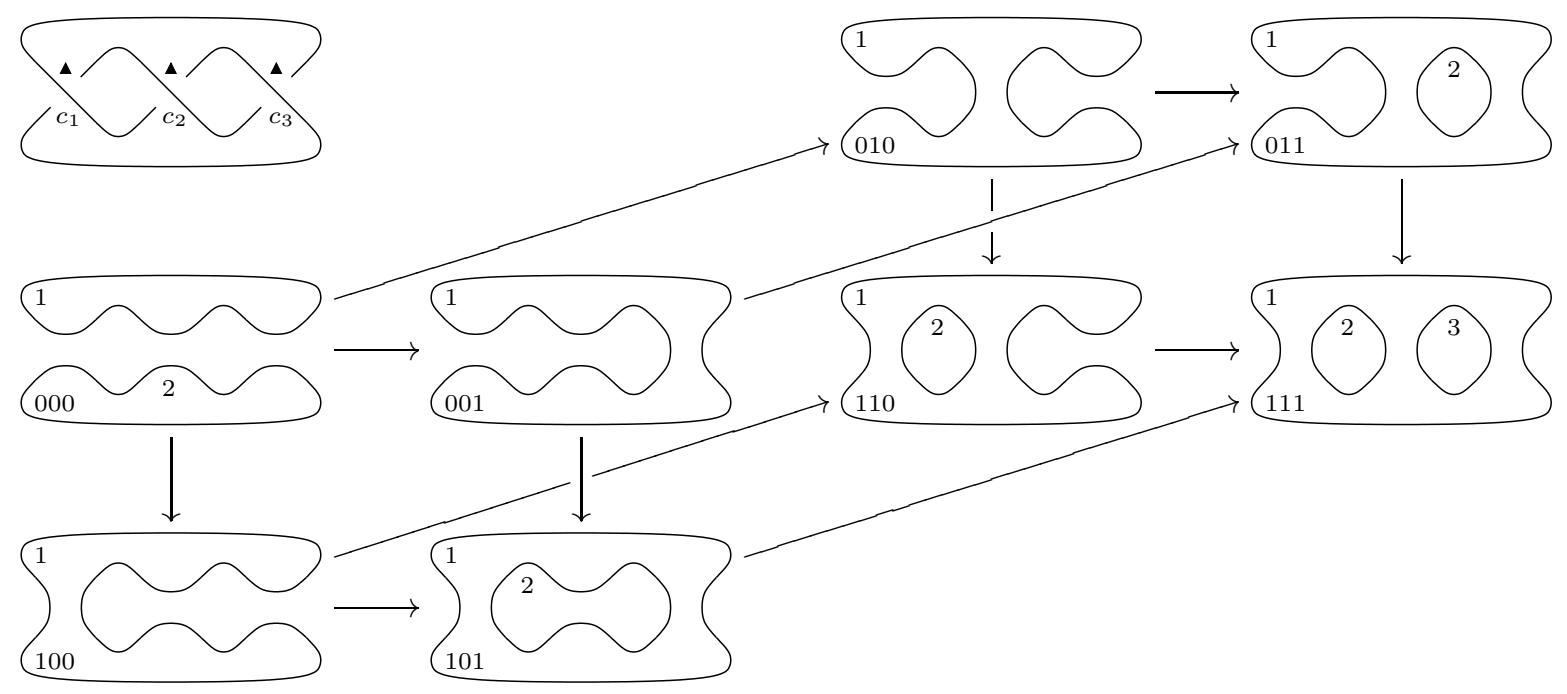

FiguRE 3.2. A decorated link diagram for the trefoil and its cube of resolutions.

Definition 3.5. Starting from a decorated link diagram, our chain complex is defined over $\mathbb{F}_{2}[H, W]$ as

$$
\mathcal{C}_{\text {tot }}=\left(\mathcal{C} \otimes \mathbb{F}_{2}[H, W], \delta_{\text {tot }}=\mathbf{d}_{1}+W \mathbf{d}_{2}+W^{2} \mathbf{d}_{3}+\cdots+H \mathbf{h}_{1}+H W \mathbf{h}_{2}+H W^{2} \mathbf{h}_{3}+\cdots\right)
$$

with $H$ and $W$ being formal variables lying in grading $(0,-2)$ and $(-1,-2)$, and the differential being of grading $(1,0)$. Setting $H=0$ recovers the Szabó geometric chain complex from Definition 2.6, while setting $W=0$ recovers the Bar-Natan chain complex from Definition 2.5. Some filtered versions and a localized version are defined as

$$
\begin{aligned}
\mathcal{C}_{f t o t} & =\mathcal{C}_{\text {tot }} /\{H=W=1\}=(\mathcal{C}, \mathbf{d}+\mathbf{h})=\left(\mathcal{C}, \mathbf{d}_{1}+\mathbf{d}_{2}+\cdots+\mathbf{h}_{1}+\mathbf{h}_{2}+\cdots\right) \\
\mathcal{C}_{f H t o t} & =\mathcal{C}_{t o t} /\{H=1\}=\left(\mathcal{C},\left(\mathbf{d}_{1}+\mathbf{h}_{1}\right)+W\left(\mathbf{d}_{2}+\mathbf{h}_{2}\right)+W^{2}\left(\mathbf{d}_{3}+\mathbf{h}_{3}\right)+\cdots\right) \\
\{H\}^{-1} \mathcal{C}_{t o t} & =\mathcal{C}_{t o t} \otimes_{\mathbb{F}_{2}[H, W]} \mathbb{F}_{2}\left[H, H^{-1}, W\right]=\left(\mathcal{C} \otimes \mathbb{F}_{2}\left[H, H^{-1}, W\right], \delta_{t o t}\right) .
\end{aligned}
$$

For a pointed decorated link diagram, $\mathcal{C}_{\text {tot }}^{-}$(respectively, $\left.\mathcal{C}_{\text {tot }}^{+}\right)$is defined to be the subcomplex (respectively, quotient complex) generated by $\mathcal{C}^{-}$(respectively, $\mathcal{C}^{+}$); they fit into a short exact sequence over $\mathbb{F}_{2}[H, W]$

$$
0 \rightarrow \mathcal{C}_{\text {tot }}^{-}\{1\} \rightarrow \mathcal{C}_{\text {tot }} \rightarrow \mathcal{C}_{\text {tot }}^{+}\{-1\} \rightarrow 0 .
$$

Example 3.1. Consider the decorated link diagram for the trefoil and its cube of resolutions in Figure 3.2. We have numbered the three crossings from left to right as $c_{1}, c_{2}, c_{3}$; the complete resolution at $u \subseteq \mathfrak{C}$ has been labeled by the corresponding vertex $\bar{u} \in\{0,1\}^{3}$, where $\bar{u}_{i}=1$ iff $c_{i} \in u$. Furthermore, the circles in each individual resolution are also numbered (usually left to right, sometimes top to bottom); let $x_{i}^{\bar{u}}$ be the circle labeled $i$ in the resolution at $u$. We will use the superscript $\bar{u}$ for the Khovanov generators living over $u$. 
The complex $\mathcal{C}_{\text {tot }}$ is freely generated over $\mathbb{F}_{2}[H, W]$ by the thirty Khovanov generators. The differential $\delta_{\text {tot }}$ is the following:

$$
\begin{aligned}
& \delta_{\text {tot }}\left(1^{000}\right)=1^{100}+1^{010}+1^{001}+W\left(1^{110}+1^{101}+1^{011}\right)+W^{2} 1^{111} \\
& \delta_{\text {tot }}\left(x_{1}^{000} x_{2}^{000}\right)=H \delta_{\text {tot }}\left(x_{1}^{000}\right)=H \delta_{\text {tot }}\left(x_{2}^{000}\right)=H\left(x_{1}^{100}+x_{1}^{010}+x_{1}^{001}\right) \\
& \delta_{\text {tot }}\left(1^{100}\right)+x_{1}^{011}+x_{2}^{011}+H 1^{011}=\delta_{\text {tot }}\left(1^{010}\right)+x_{1}^{101}+x_{2}^{101}+H 1^{101}=\delta_{\text {tot }}\left(1^{001}\right)+x_{1}^{110}+x_{2}^{110}+H 1^{110} \\
& \quad=x_{1}^{110}+x_{2}^{110}+x_{1}^{101}+x_{2}^{101}+x_{1}^{011}+x_{2}^{011}+H\left(1^{110}+1^{101}+1^{011}\right)+H W 1^{111} \\
& \delta_{\text {tot }}\left(x_{1}^{100}\right)+x_{1}^{011} x_{2}^{011}=\delta_{\text {tot }}\left(x_{1}^{010}\right)+x_{1}^{101} x_{2}^{101}=\delta_{\text {tot }}\left(x_{1}^{001}\right)+x_{1}^{110} x_{2}^{110}=x_{1}^{110} x_{2}^{110}+x_{1}^{101} x_{2}^{101}+x_{1}^{011} x_{2}^{011} \\
& \delta_{\text {tot }}\left(1^{110}\right)+x_{2}^{111}=\delta_{\text {tot }}\left(1^{101}\right)+x_{1}^{111}=\delta_{\text {tot }}\left(1^{011}\right)+x_{3}^{111}=x_{1}^{111}+x_{2}^{111}+x_{3}^{111}+H 1^{111} \\
& \delta_{\text {tot }}\left(x_{1}^{110}\right)+x_{1}^{111} x_{3}^{111}=\delta_{\text {tot }}\left(x_{2}^{101}\right)+x_{2}^{111} x_{3}^{111}=\delta_{\text {tot }}\left(x_{1}^{011}\right)+x_{1}^{111} x_{2}^{111}=0 \\
& \delta_{\text {tot }}\left(x_{2}^{110}\right)+x_{1}^{111} x_{3}^{111}+H x_{2}^{111}=\delta_{\text {tot }}\left(x_{1}^{101}\right)+x_{2}^{111} x_{3}^{111}+H x_{1}^{111}=\delta_{\text {tot }}\left(x_{2}^{011}\right)+x_{1}^{111} x_{2}^{111}+H x_{3}^{111} \\
& \quad=x_{1}^{111} x_{2}^{111}+x_{1}^{111} x_{3}^{111}+x_{2}^{111} x_{3}^{111} \\
& \delta_{\text {tot }}\left(x_{1}^{110} x_{2}^{110}\right)=\delta_{\text {tot }}\left(x_{1}^{101} x_{2}^{101}\right)=\delta_{\text {tot }}\left(x_{1}^{011} x_{2}^{011}\right)=x_{1}^{111} x_{2}^{111} x_{3}^{111} ;
\end{aligned}
$$

and it is zero on the other (eight) Khovanov generators.

We will now spend the rest of the section working towards a proof of Proposition 3.4. Towards that end, we construct a decomposition of $\mathbf{h}$, generalize the notion of trees and dual trees, and prove some relevant lemmas.

Definition 3.6. For each non-empty subset $\varnothing \neq s \subseteq \mathfrak{C}$, define the following endomorphism $\mathbf{h}_{s}$ of $\mathcal{C}$.

$$
\left\langle\mathbf{h}_{s}((u, x)),(v, y)\right\rangle= \begin{cases}\langle\mathbf{h}((u, x)),(v, y)\rangle & \text { if } v=u \amalg s, \\ 0 & \text { otherwise. }\end{cases}
$$

It is clear that $\mathbf{h}=\sum_{\varnothing \neq s \subseteq \mathfrak{C}} \mathbf{h}_{s} ;$ indeed, $\mathbf{h}_{i}=\sum_{\substack{s \subseteq \mathfrak{C} \\|s|=i}} \mathbf{h}_{s}$.

Definition 3.7. In a similar vein to Definition 3.1, let a potted tree denote a part of a labeled resolution configuration consisting of $k$ initial circles and $(k-1)$ arcs that are arranged as a tree (with $k \geq 1$ ), such that there is exactly one additional arc joining these $k$ circles to the rest of the resolution configuration; furthermore, all these $k$ circles appear in the starting monomial, and the unique ending circle they correspond to, appears in the ending monomial. A potted dual tree is part of a labeled resolution configuration whose dual is a potted tree.

Lemma 3.8. For any non-empty subsets $s, t$ of $\mathfrak{C}$,

$$
\mathbf{h}_{s} \mathbf{h}_{t}= \begin{cases}\mathbf{h}_{s} \amalg^{t} & \text { if } s \bigcap t=\varnothing, \\ 0 & \text { otherwise. }\end{cases}
$$

Proof. We want to show $\left\langle\mathbf{h}_{s} \mathbf{h}_{t}((u, x)),(v, y)\right\rangle=\left\langle\mathbf{h}_{s} \amalg t((u, x)),(v, y)\right\rangle$ for all disjoint non-empty subsets $s, t$, and for all Khovanov generators $(u, x)$ and $(v, y)$. Either can be non-zero only if $v=u \amalg s \coprod t$, so we may assume $u$ is disjoint from $s$ and $t$, and $v=u \amalg s \coprod t$. Let $w=u \coprod t$. 
The contribution $\left\langle\mathbf{h}_{s} \amalg t((u, x)),(v, y)\right\rangle$ is non-zero if and only if $\left(D_{u}^{v}, x, y\right)$ is a disjoint union of trees and dual trees. Since graph minors of forests are also forests, for a unique monomial $z$ on $Z\left(D_{w}\right)$, both $\left(D_{u}^{w}, x, z\right)$ and $\left(D_{w}^{v}, z, y\right)$ are disjoint unions of trees and dual trees. Therefore, $\mathbf{h}_{t}((u, x))=(w, z)$ and $\mathbf{h}_{s}((w, z))=(v, y)$, and hence $\left\langle\mathbf{h}_{s} \mathbf{h}_{t}((u, x)),(v, y)\right\rangle \neq 0$.

Conversely, if $\left\langle\mathbf{h}_{s} \mathbf{h}_{t}((u, x)),(v, y)\right\rangle \neq 0$, let $(w, z)=\mathbf{h}_{t}((u, x))$ (note, $\mathbf{h}_{t}$ evaluated on a Khovanov generator can have at most one non-zero term). Then both $\left(D_{u}^{w}, x, z\right)$ and $\left(D_{w}^{v}, z, y\right)$ are disjoint unions of trees and dual trees. In other words, the resolution configuration $\left(D_{u}^{v}, x, y\right)$ is obtained from $\left(D_{u}^{w}, x, z\right)$ by adding a bunch of potted trees and potted dual trees. Therefore, $\left(D_{u}^{v}, x, y\right)$ is a disjoint union of trees and dual trees as well, and hence $\left\langle\mathbf{h}_{s} \amalg t((u, x)),(v, y)\right\rangle \neq 0$.

Corollary 3.9. For any non-empty subsets $s, t$ of $\mathfrak{C}$,

$$
\left[\mathbf{h}_{s}: \mathbf{h}_{t}\right]=0
$$

Therefore, for any non-empty subset $s$ of $\mathfrak{C},\left[\mathbf{h}_{s}: \mathbf{h}\right]=0 ;$ and $\mathbf{h}^{2}=0$.

Lemma 3.10. Let $D$ and $D^{\prime}$ be two decorated link diagrams, which differ in the choice of decorations at precisely the crossings in some subset $\mathfrak{S} \subseteq \mathfrak{C}$, but are otherwise identical. Let $\mathbf{d}$ and $\mathbf{d}^{\prime}$ be the respective endomorphisms of $\mathcal{C}$ defined via the contribution function $\mathfrak{d}$. Then,

$$
\mathbf{d}^{\prime}=\mathbf{d}+\sum_{\varnothing \neq s \subseteq \mathfrak{S}}\left[\mathbf{h}_{s}: \mathbf{d}\right]+\sum_{\substack{\varnothing \neq s, t \subseteq \mathfrak{S} \\ s \cap t=\varnothing}} \mathbf{h}_{s} \mathbf{d} \mathbf{h}_{t}=\mathbf{d}+\sum_{\varnothing \neq s \subseteq \mathfrak{S}}\left[\mathbf{h}_{s}: \mathbf{d}\right]+\sum_{\varnothing \neq s, t \subseteq \mathfrak{S}} \mathbf{h}_{s} \mathbf{d} \mathbf{h}_{t} .
$$

Proof. The second and the third expressions agree because $\mathbf{h}_{s} \mathbf{d} \mathbf{h}_{t}=0$ if $s \bigcap t \neq \varnothing$. Therefore, it is enough to prove that the first and the second expressions agree. We do it by induction on the number of elements in $\mathfrak{S}$.

The base case, when $\mathfrak{S}$ consists of a single crossing, is essentially [Szab, Theorem 5.4]. So we only need the induction step. Let $D^{\prime \prime}$ be the decorated link diagram obtained from $D$ by changing the decoration at a single crossing $c \in \mathfrak{S}$, and let $\mathbf{d}^{\prime \prime}$ be the corresponding endomorphism. From the base case,

$$
\mathbf{d}^{\prime \prime}=\mathbf{d}+\left[\mathbf{h}_{\{c\}}: \mathbf{d}\right]
$$

and from the induction step,

$$
\mathbf{d}^{\prime}=\mathbf{d}^{\prime \prime}+\sum_{\varnothing \neq s \subseteq \mathfrak{S} \backslash\{c\}}\left[\mathbf{h}_{s}: \mathbf{d}^{\prime \prime}\right]+\sum_{\substack{\varnothing \neq s, t \subseteq \mathfrak{S} \backslash\{c\} \\ s \cap t=\varnothing}} \mathbf{h}_{s} \mathbf{d}^{\prime \prime} \mathbf{h}_{t}
$$


Combining (and using Lemma 3.8), we get

$$
\begin{aligned}
& \mathbf{d}^{\prime}=\mathbf{d}+\left[\mathbf{h}_{\{c\}}: \mathbf{d}\right]+\sum_{\varnothing \neq s \subseteq \mathfrak{S} \backslash\{c\}}\left[\mathbf{h}_{s}: \mathbf{d}+\left[\mathbf{h}_{\{c\}}: \mathbf{d}\right]\right]+\sum_{\substack{\varnothing \neq s, t \subseteq \mathfrak{S} \backslash\{c\} \\
s \cap t=\varnothing}} \mathbf{h}_{s}\left(\mathbf{d}+\left[\mathbf{h}_{\{c\}}: \mathbf{d}\right]\right) \mathbf{h}_{t} \\
& =\mathbf{d}+\left[\mathbf{h}_{\{c\}}: \mathbf{d}\right]+\sum_{\varnothing \neq s \subseteq \mathfrak{S} \backslash\{c\}}\left(\left[\mathbf{h}_{s}: \mathbf{d}\right]+\left[\mathbf{h}_{s} \amalg\{c\}: \mathbf{d}\right]\right)+\sum_{\varnothing \neq s \subseteq \mathfrak{S} \backslash\{c\}}\left(\mathbf{h}_{s} \mathbf{d} \mathbf{h}_{\{c\}}+\mathbf{h}_{\{c\}} \mathbf{d} \mathbf{h}_{s}\right) \\
& +\sum_{\substack{\varnothing \neq s, t \subseteq \mathfrak{S} \backslash\{c\} \\
s \cap \bar{t}=\varnothing}}\left(\mathbf{h}_{s} \mathbf{d} \mathbf{h}_{t}+\mathbf{h}_{s} \amalg\{c\} \mathbf{d} \mathbf{h}_{t}+\mathbf{h}_{s} \mathbf{d} \mathbf{h}_{t} \amalg\{c\}\right) \\
& =\mathbf{d}+\sum_{\varnothing \neq s \subseteq \mathfrak{S}}\left[\mathbf{h}_{s}: \mathbf{d}\right]+\sum_{\substack{\varnothing \neq s, t \subseteq \mathfrak{S} \\
s \cap t=\varnothing}} \mathbf{h}_{s} \mathbf{d} \mathbf{h}_{t} .
\end{aligned}
$$

Lemma 3.11. Let $D$ and $D^{\prime}$ be two decorated link diagrams, which might differ in the choice of decorations, but are otherwise identical. Let $\mathbf{d}$ and $\mathbf{d}^{\prime}$ be the respective endomorphisms of $\mathcal{C}$. Fix $k \geq 1$. If

$$
\langle[\mathbf{h}: \mathbf{d}]((u, x)),(v, y)\rangle=0
$$

for all pairs of Khovanov generators $(u, x)$ and $(v, y)$ with $|v|-|u|<k$, then

$$
\langle[\mathbf{h}: \mathbf{d}]((u, x)),(v, y)\rangle=\left\langle\left[\mathbf{h}: \mathbf{d}^{\prime}\right]((u, x)),(v, y)\right\rangle
$$

for all pairs of Khovanov generators $(u, x)$ and $(v, y)$ with $|v|-|u|=k$.

Proof. Let $\mathfrak{S}$ be the set of crossings where the decorations of $D$ and $D^{\prime}$ differ. By Lemma 3.10,

$$
\mathbf{d}^{\prime}-\mathbf{d}=\sum_{\varnothing \neq s \subseteq \mathfrak{S}}\left[\mathbf{h}_{s}: \mathbf{d}\right]+\sum_{\varnothing \neq s, t \subseteq \mathfrak{S}} \mathbf{h}_{s} \mathbf{d} \mathbf{h}_{t},
$$

and hence (with the aid of Corollary 3.9),

$$
\begin{aligned}
{\left[\mathbf{h}: \mathbf{d}^{\prime}-\mathbf{d}\right] } & =\sum_{\varnothing \neq s \subseteq \mathfrak{S}}\left[\mathbf{h}:\left[\mathbf{h}_{s}: \mathbf{d}\right]\right]+\sum_{\varnothing \neq s, t \subseteq \mathfrak{S}}\left[\mathbf{h}: \mathbf{h}_{s} \mathbf{d} \mathbf{h}_{t}\right] \\
& =\sum_{\varnothing \neq s \subseteq \mathfrak{S}}\left[\mathbf{h}_{s}:[\mathbf{h}: \mathbf{d}]\right]+\sum_{\varnothing \neq s, t \subseteq \mathfrak{S}} \mathbf{h}_{s}[\mathbf{h}: \mathbf{d}] \mathbf{h}_{t} .
\end{aligned}
$$


Therefore, for any $(u, x)$ and $(v, y)$ with $\operatorname{gr}_{h}((v, y))-\operatorname{gr}_{h}((u, x))=|v|-|u|=k$,

$$
\begin{aligned}
&\left\langle\left[\mathbf{h}: \mathbf{d}^{\prime}-\mathbf{d}\right]((u, x)),(v, y)\right\rangle= \sum_{\varnothing \neq s \subseteq \mathfrak{S}}\left\langle\left[\mathbf{h}_{s}:[\mathbf{h}: \mathbf{d}]\right]((u, x)),(v, y)\right\rangle \\
&+\sum_{\substack{\varnothing \neq s, t \subseteq \mathfrak{S} \\
=}}\left\langle\mathbf{h}_{s}[\mathbf{h}: \mathbf{d}] \mathbf{h}_{t}((u, x)),(v, y)\right\rangle \\
& \sum_{\substack{s,(w, z) \\
\varnothing \neq s \subseteq \mathfrak{S} \\
|w|-|u|=k-|s|}}\left\langle\mathbf{h}_{s}((w, z)),(v, y)\right\rangle\langle[\mathbf{h}: \mathbf{d}]((u, x)),(w, z)\rangle \\
&+\sum_{\substack{s,(w, z) \\
\varnothing \neq s \subseteq \mathfrak{S} \\
|v|-|w|=k-|s|}}\langle[\mathbf{h}: \mathbf{d}]((w, z)),(v, y)\rangle\left\langle\mathbf{h}_{s}((u, x)),(w, z)\right\rangle \\
&+\sum_{\substack{\left\langle\mathbf{h}_{s} \\
s, t,\left(w_{1}, z_{1}\right),\left(w_{2}, z_{2}\right) \\
\varnothing \neq s, t \subseteq \mathfrak{S} \\
\left|w_{1}\right|-|u|=|t|\right.}}^{\left|w_{2}\right|-\left|w_{1}\right|=k-|s|-|t|} \\
&=0
\end{aligned}
$$

by the hypothesis.

Let us now define five families of labeled resolution configurations that will be of relevance very soon.

Definition 3.12. A Type- $\alpha$ configuration (Figure 3.3a after ignoring the orientations) is obtained from a Type-A configuration by forgetting the orientation of the arcs, and adding some number (possibly zero) of potted dual trees on the two initial circles. A Type- $\beta$ configuration (Figure 3.3b after ignoring the orientations) is the dual of a Type- $\alpha$ configuration; therefore, it is obtained from a Type-B configuration by forgetting the orientation of the arcs, and adding potted trees on the initial circles. A Type- $\gamma$ configuration (Figure 3.3c after ignoring the orientations) is obtained from a Type-C configuration by forgetting the orientation of the arcs, and adding potted dual trees on the initial circle. A Type- $\delta$ configuration (Figure 3.3d after ignoring the orientations) is the dual of a Type- $\gamma$ configuration; therefore, it is obtained from a Type-D configuration by forgetting the orientation of the arcs, and adding potted trees on the initial circles. A Type- $\varepsilon$ configuration (Figure 3.3e after ignoring the orientations) is obtained by adding potted trees and potted dual trees on a single circle, and requiring that the index of the resulting resolution configuration be at least one.

Lemma 3.13. If $(u, x),(v, y)$ and $(w, z)$ are Khovanov generators such that $\langle\mathbf{d}((u, x)),(w, z)\rangle \neq 0$ and $\langle\mathbf{h}((w, z)),(v, y)\rangle \neq 0$, then, $\left(D_{u}^{v}, x, y\right)$, after forgetting the orientations of the arcs, is equivalent to a disjoint union of trees, dual trees and exactly one configuration from one of the five families in Definition 3.12. 


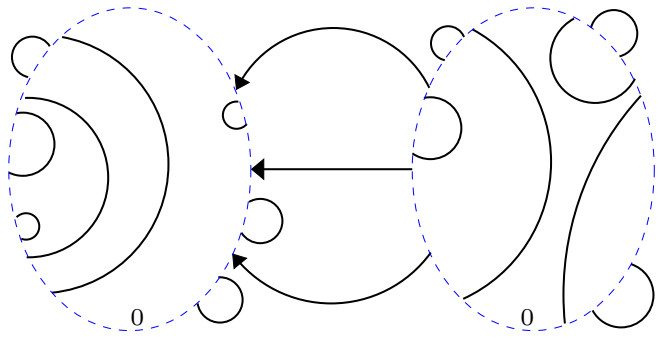

a: Type- $\alpha$ configuration.

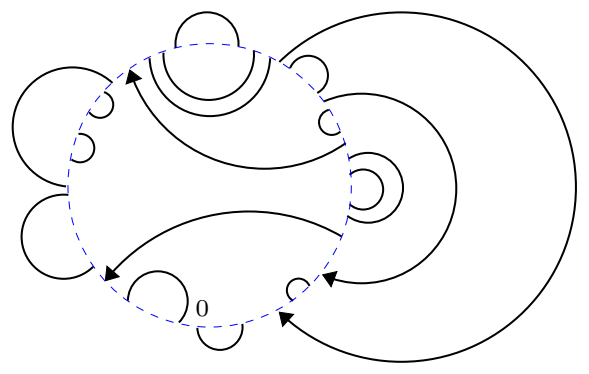

c: Type- $\gamma$ configuration.

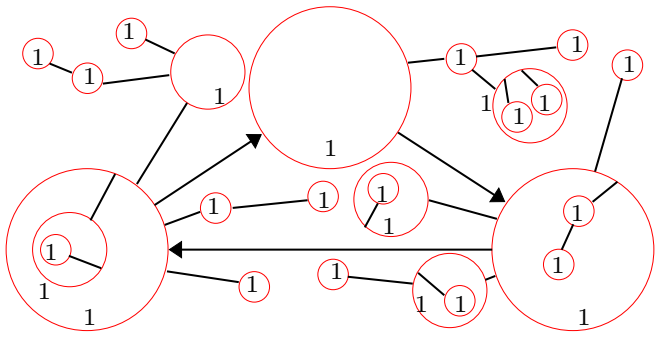

b: Type- $\beta$ configuration.

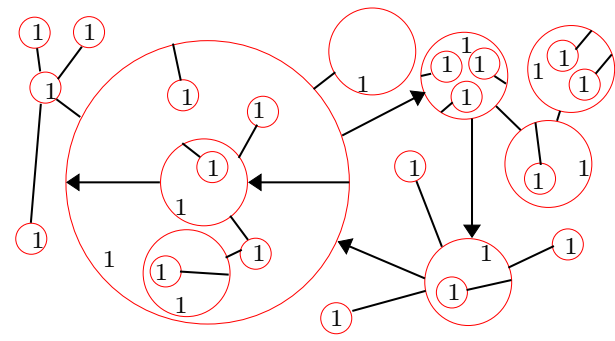

d: Type- $\delta$ configuration.

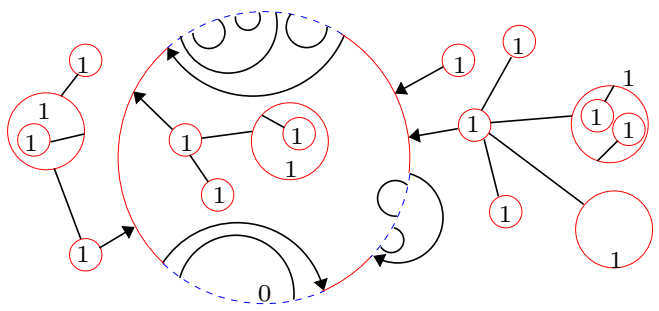

e: Type- $\varepsilon$ configuration.

FiguRE 3.3. After ignoring the orientations of the arcs, these are the labeled resolution configurations from Definition 3.12.

Proof. The proof is a fairly straightforward case analysis. Since $\langle\mathbf{d}((u, x)),(w, z)\rangle \neq 0$, the active part of the resolution configuration $\left(D_{u}^{w}, x, z\right)$ must be in one of the five families described in Section 3 or Figure 2.6, and since $\langle\mathbf{h}((w, z)),(v, y)\rangle \neq 0$, the resolution configuration $\left(D_{w}^{v}, z, y\right)$ must be a disjoint union of trees and dual trees. Therefore, we need to show that the union of the following is a configuration from one of the five families in Definition 3.12:

(i) the active part of $\left(D_{u}^{w}, x, z\right)$;

(ii) the arcs of $D_{w}^{v}$ that are in the same connected component (of $D_{w}^{v}$ ) as some active ending circle of $D_{u}^{w}$; and

(iii) the starting circles of $\left(D_{w}^{v}, z, y\right)$ that are passive as ending circles of $D_{u}^{w}$ but are in the same connected component (of $D_{w}^{v}$ ) as some active ending circle of $D_{u}^{w}$. 
If the active part of $\left(D_{u}^{w}, x, z\right)$ is a Type-A configuration, then adding the relevant portion from $\left(D_{w}^{v}, z, y\right)$ has the effect of adding some (possibly zero) more parallel arcs between the two active starting circles of $D_{u}^{w}$, followed by adding some potted dual trees; consequently, we get a Type- $\alpha$ configuration.

If the active part of $\left(D_{u}^{w}, x, z\right)$ is a Type-B configuration, then adding the relevant portion from $\left(D_{w}^{v}, z, y\right)$ could achieve one of the following two things. If the new arcs and circles do not connect the two active ending circles of $D_{u}^{w}$, then we get a Type- $\beta$ configuration; and if the new arcs and circles do connect the two active ending circles of $D_{u}^{w}$, then we get a Type- $\delta$ configuration.

If the active part of $\left(D_{u}^{w}, x, z\right)$ is a Type-C configuration, then adding the relevant portion from $\left(D_{w}^{v}, z, y\right)$ has the effect of adding some (possibly zero) number of arcs parallel to inside or outside arcs of $D_{u}^{w}$, followed by adding some potted dual trees; therefore, we get a Type- $\gamma$ configuration.

If the active part of $\left(D_{u}^{w}, x, z\right)$ is a Type-D configuration, then adding the relevant portion from $\left(D_{w}^{v}, z, y\right)$ is simply the addition of some potted trees, and we get a Type- $\delta$ configuration.

Finally, if the active part of $\left(D_{u}^{w}, x, z\right)$ is a Type-E configuration, then adding the relevant portion from $\left(D_{w}^{v}, z, y\right)$ is same as adding some potted trees and potted dual trees, and therefore, we get a Type- $\varepsilon$ configuration.

Lemma 3.14. If $(u, x),(v, y)$ and $(w, z)$ are Khovanov generators such that $\langle\mathbf{h}((u, x)),(w, z)\rangle \neq 0$ and $\langle\mathbf{d}((w, z)),(v, y)\rangle \neq 0$, then, $\left(D_{u}^{v}, x, y\right)$, after forgetting the orientations of the arcs, is equivalent to a disjoint union of trees, dual trees and exactly one configuration from one of the five families in Definition 3.12.

Proof. This follows immediately from Lemma 3.13. Since both the contribution functions $\mathfrak{d}$ and $\mathfrak{h}$ satisfy the duality rule, the resolution configuration $\left(D_{u}^{v}, x, y\right)$ must be dual to some resolution configuration from Lemma 3.13. Therefore, it must be dual to a disjoint union of trees, dual trees, and exactly one configuration from Definition 3.12. However, the resolution configurations from Definition 3.12 are closed under taking duals; namely, Type- $\alpha$ configurations are dual to Type- $\beta$ configurations, Type- $\gamma$ configurations are dual to Type- $\delta$ configurations, and Type- $\varepsilon$ configurations are self-dual.

We are finally ready to embark upon the proof of our main result for this section.

Proof of Proposition 3.4. We want to show $(\mathbf{d}+\mathbf{h})^{2}=0$ on $\mathcal{C}$. Since $\mathbf{d}$ increases $\operatorname{gr}_{\delta}$ by one, and $\mathbf{h}$ preserves it, this is equivalent to the following three statements, arranged in the increasing order of difficulty:

$$
\mathbf{h}^{2}=0 \quad[\mathbf{h}: \mathbf{d}]=0 \quad \mathbf{d}^{2}=0 .
$$

Corollary 3.9 already takes care of the easiest of these three statements; and the hardest is proved in $[$ Szab, Section 6]. Therefore, we will merely prove $[\mathbf{h : ~} \mathbf{d}]=0$. Certain formulas for certain counts take a simpler form if we write them for the equivalent statement

$$
[\mathbf{h}+\mathrm{Id}: \mathbf{d}]=0,
$$

and so that is what we will prove.

We prove the above equation by induction on the index. The base case when the index is zero, is vacuous; hence we only prove the induction step. We assume

$$
\langle[\mathbf{h}+\mathrm{Id}: \mathbf{d}]((u, x)),(v, y)\rangle=0
$$


for all pairs of Khovanov generators $(u, x)$ and $(v, y)$ with $\operatorname{gr}_{h}((v, y))-\operatorname{gr}_{h}((u, x))<k$, and for any decoration of the original link diagram $D$. Then fix Khovanov generators $(u, x)$ and $(v, y)$ with $u \subset v$ and $\operatorname{gr}_{h}((v, y))-\operatorname{gr}_{h}((u, x))=k$, and fix some decoration on $D$. We will prove that

$$
\langle[\mathbf{h}+\mathrm{Id}: \mathbf{d}]((u, x)),(v, y)\rangle=0
$$

holds for that decoration.

Thanks to Lemma 3.11, it is enough to prove this for some decoration; that is, after fixing $(u, x)$ and $(v, y)$, we are free to choose our decoration. By Lemma 3.13 and Lemma 3.14, we may assume that $\left(D_{u}^{v}, x, y\right)$ is a disjoint union of trees, dual trees, and exactly one configuration from Definition 3.12. These configurations are shown in Figure 3.3, with some of the arcs being oriented; assume the number of oriented arcs is $\ell$. Choose some decoration on $D$ so that those $\ell$ arcs are oriented as in Figure 3.3. We will finish the proof by analyzing each of the configurations from Figure 3.3.

If $\left(D_{u}^{v}, x, y\right)$ is equivalent to a disjoint union of trees, dual trees and the configuration from Figure 3.3a, then it breaks up in the following ways:

- We first use all the arcs except the $\ell$ oriented ones to get a configuration that contributes to $(\mathbf{h}+\mathrm{Id})$; and we follow it by the $\ell$ oriented arcs to get a Type-A configuration which contributes to $\mathbf{d}$.

- We use a non-empty subset of the $\ell$ oriented arcs to get a Type-A configuration that contributes to $\mathbf{d}$; and we follow it by the rest of the arcs to get a configuration that contributes to $(\mathbf{h}+\mathrm{Id})$.

Therefore, we get a total of $1+\left(2^{\ell}-1\right)$ contributions to $[\mathbf{h}: \mathbf{d}]$, and hence are done with this case.

If $\left(D_{u}^{v}, x, y\right)$ is equivalent to a disjoint union of trees, dual trees and the configuration from Figure $3.3 \mathrm{~b}$, then it is dual to the previous case, and therefore follows from the previous case since all the relevant contribution functions satisfy the duality rule.

Next assume that $\left(D_{u}^{v}, x, y\right)$ is equivalent to a disjoint union of trees, dual trees and the configuration from Figure 3.3c; assume the number of oriented arcs inside (respectively, outside) the starting circle is $\ell_{1}$ (respectively, $\ell_{2}$ ) with $\ell=\ell_{1}+\ell_{2}$. The configuration breaks up in the following ways:

- We first use all except the $\ell_{1}$ oriented arcs inside to get a configuration that contributes to $(\mathbf{h}+\mathrm{Id})$; and we follow it by the $\ell_{1}$ oriented arcs to get a Type-A configuration that contributes to $\mathbf{d}$.

- We first use all except the $\ell_{2}$ oriented arcs outside to get a configuration that contributes to $(\mathbf{h}+\mathrm{Id})$; and we follow it by the $\ell_{2}$ oriented arcs to get a Type-A configuration that contributes to $\mathbf{d}$.

Note, we have chosen the orientations of the $\ell$ arcs in such a way that we do not have to encounter Type-C configurations. Therefore, we get a total of $1+1$ contributions to $[\mathbf{h}: \mathbf{d}]$, and once again we are done.

If $\left(D_{u}^{v}, x, y\right)$ is equivalent to a disjoint union of trees, dual trees and the configuration from Figure 3.3d, then it is dual to the previous case, and therefore follows from it.

Finally assume that $\left(D_{u}^{v}, x, y\right)$ is equivalent to a disjoint union of trees, dual trees and the configuration from Figure 3.3e. Assume the number of potted trees on the special starting circle is 
$\ell_{1}$, and the number of potted dual trees on the special starting circle is $\ell_{2}$, with $\ell_{1}+\ell_{2}=\ell \geq 1$. There are the following subcases.

(1) $\ell_{1} \neq 0, \ell_{2}=0$. The configuration breaks up in the following ways:

- We first use all except the $\ell_{1}$ oriented arcs to get a configuration that contributes to $(\mathbf{h}+\mathrm{Id})$; and we follow it by the $\ell_{1}$ oriented arcs to get a Type-E configuration that contributes to $\mathbf{d}$.

- We use a non-empty subset of the $\ell_{1}$ oriented arcs to get a Type-E configuration that contributes to $\mathbf{d}$; and we follow it by the rest of the arcs to get a configuration that contributes to $(\mathbf{h}+\mathrm{Id})$.

(2) $\ell_{1}=0, \ell_{2} \neq 0$. The configuration breaks up in the following ways:

- We first use all the $\ell_{2}$ oriented arcs to get a Type-E configuration that contributes to $\mathbf{d}$; and we follow it by the rest of the arcs to get a configuration that contributes to $(\mathbf{h}+\mathrm{Id})$.

- We use all the non-oriented arcs and a proper subset of the $\ell_{2}$ oriented arcs to get a configuration that contributes to $(\mathbf{h}+\mathrm{Id})$; and we follow it by the remaining arcs to get a Type-E configuration that contributes to $\mathbf{d}$.

(3) $\ell_{1} \neq 0, \ell_{2} \neq 0$. The configuration breaks up in the following ways:

- We use all the non-oriented arcs and all of the $\ell_{2}$ oriented arcs to get a configuration that contributes to $(\mathbf{h}+\mathrm{Id})$; and we follow it by the rest of the arcs to get a Type-E configuration that contributes to $\mathbf{d}$.

- We use all the $\ell_{2}$ oriented arcs to get a Type-E configuration that contributes to $\mathbf{d}$; and we follow it by the rest of the arcs to get a configuration that contributes to $(\mathbf{h}+\mathrm{Id})$.

Therefore, we have the following number of contributions to $[\mathbf{h}: \mathbf{d}]$,

\begin{tabular}{c|cc} 
& $\ell_{1}=0$ & $\ell_{1} \geq 1$ \\
\hline$\ell_{2}=0$ & & $1+\left(2^{\ell_{1}}-1\right)$ \\
$\ell_{2} \geq 1$ & $1+\left(2^{\ell_{2}}-1\right)$ & 2
\end{tabular}

thus concluding the proof.

\section{INVARIANCE}

We now turn to invariance. The master invariant that we study is the bigraded chain homotopy type of the inclusion $\iota: \mathcal{C}_{\text {tot }}^{-}\{1\} \hookrightarrow \mathcal{C}_{\text {tot }}$ over the ring $\mathbb{F}_{2}[H, W]$. All the other variants that we have considered can easily be recovered from this variant. For example, if one wishes to recover the plus version of the filtered Szabó geometric chain complex, one can obtain it by taking the mapping cone of the inclusion $\mathcal{C}_{\text {tot }}^{-}\{1\} \hookrightarrow \mathcal{C}_{\text {tot }}$, then setting $H=0$ and $W=1$, and then shifting the quantum grading by 1.

$$
\mathcal{C}_{S z}^{+} \simeq \operatorname{Cone}(\iota) /\{H=0, W=1\}\{1\}
$$

Definition 4.1. Consider the bigraded polynomial ring $\mathbb{F}_{2}[H, W]$ with $H$ and $W$ in bigradings $(0,-2)$ and $(-1,-2)$ respectively. Let $\operatorname{Kom}\left(\mathbb{F}_{2}[H, W]\right)$ be the category of chain complexes over $\mathbb{F}_{2}[H, W]$. The objects are bigraded chain complexes over $\mathbb{F}_{2}[H, W]$ with the differentials in bigrading $(1,0)$, and the morphisms are $(0,0)$-graded $\mathbb{F}_{2}[H, W]$-module chain maps. For $C \in$ 
$\mathrm{Ob}\left(\operatorname{Kom}\left(\mathbb{F}_{2}[H, W]\right)\right.$ and integer $a$, define $C\{a\} \in \mathrm{Ob}\left(\operatorname{Kom}\left(\mathbb{F}_{2}[H, W]\right)\right.$ by shifting the second grading by $a$.

Let $\mathcal{K}\left(\mathbb{F}_{2}[H, W]\right)$ be the homotopy category over $\mathbb{F}_{2}[H, W]$. The objects are same as the objects of $\operatorname{Kom}\left(\mathbb{F}_{2}[H, W]\right)$. The morphisms are equivalence classes of morphisms in $\operatorname{Kom}\left(\mathbb{F}_{2}[H, W]\right)$; we declare $f_{1}, f_{2} \in \operatorname{Hom}_{\operatorname{Kom}\left(\mathbb{F}_{2}[H, W]\right)}(A, B)$ equivalent if there is a $(-1,0)$-graded $\mathbb{F}_{2}[H, W]$-module map $h: A \rightarrow B$ such that

$$
f_{1}-f_{2}=d_{B} \circ h+h \circ d_{A} .
$$

Let $\mathcal{K}_{\mathrm{p}}\left(\mathbb{F}_{2}[H, W]\right)$ be the homotopy category of pairs over $\mathbb{F}_{2}[H, W]$, defined as follows. The objects are triples $(A, B, \phi)$ where $A, B \in \mathrm{Ob}_{\mathrm{Kom}\left(\mathbb{F}_{2}[H, W]\right)}$, and $\phi \in \operatorname{Hom}_{\mathrm{Kom}\left(\mathbb{F}_{2}[H, W]\right)}(A, B)$. Morphisms from $\left(A_{1}, B_{1}, \phi_{1}\right)$ to $\left(A_{2}, B_{2}, \phi_{2}\right)$ are pairs $(f, g)$, where $f \in \operatorname{Hom}_{\operatorname{Kom}\left(\mathbb{F}_{2}[H, W]\right)}\left(A_{1}, A_{2}\right)$ and $g \in \operatorname{Hom}_{\mathrm{Kom}\left(\mathbb{F}_{2}[H, W]\right)}\left(B_{1}, B_{2}\right)$ such that the following commutes

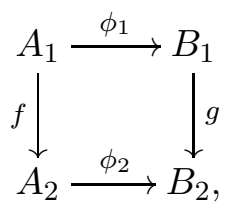

modulo the following equivalence relation. We declare $\left(f_{1}, g_{1}\right)$ equivalent to $\left(f_{2}, g_{2}\right)$ if there are $(-1,0)$-graded $\mathbb{F}_{2}[H, W]$-module maps $h: A_{1} \rightarrow A_{2}$ and $k: B_{1} \rightarrow B_{2}$ such that the following commutes

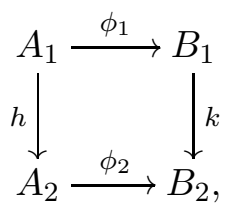

and

$$
\begin{aligned}
& f_{1}-f_{2}=d_{A_{2}} \circ h+h \circ d_{A_{1}} \\
& g_{1}-g_{2}=d_{B_{2}} \circ h+h \circ d_{B_{1}} .
\end{aligned}
$$

Proposition 4.2. Let $(D, p)$ and $\left(D^{\prime}, p^{\prime}\right)$ be two pointed decorated link diagrams representing isotopic pointed links in $S^{3}$. Then the objects $\left(\mathcal{C}_{\text {tot }}^{-}(D, p)\{1\}, \mathcal{C}_{\text {tot }}(D), \iota(D, p)\right)$ and $\left(\mathcal{C}_{\text {tot }}^{-}\left(D^{\prime}, p^{\prime}\right)\{1\}\right.$, $\left.\mathcal{C}_{\text {tot }}\left(D^{\prime}\right), \iota\left(D^{\prime}, p^{\prime}\right)\right)$ are isomorphic in the homotopy category of pairs $\mathcal{K}_{\mathrm{p}}\left(\mathbb{F}_{2}[H, W]\right)$.

Corollary 4.3. Let $D$ and $D^{\prime}$ be two decorated link diagrams representing isotopic links in $S^{3}$. Then the objects $\mathcal{C}_{\text {tot }}(D)$ and $\mathcal{C}_{\text {tot }}\left(D^{\prime}\right)$ are isomorphic in the homotopy category $\mathcal{K}\left(\mathbb{F}_{2}[H, W]\right)$.

We will basically check invariance under the three Reidemeister moves from Figure 4.1, following the standard arguments. Along the way, we will need the following well-known (and heavily used) cancellation principle.

Definition 4.4. Fix a link diagram $D$. A 5 -tuple $\left(\mathfrak{C}_{0}, u, v, c, a\right)$ is called a cancellation data if $u \subsetneq\{c\} \cup u=v \subseteq \mathfrak{C}_{0} \subseteq \mathfrak{C}$, and one of the following holds.

(1) The surgery arc $\alpha_{c}$ joins two different circles in $D_{u}$, the complete resolution of $D$ corresponding to $u$, and $a \in Z\left(D_{u}\right)$ is one of the two circles connected by $\alpha_{c}$, and $a$ is disjoint 
from $\alpha_{c^{\prime}}$ for all $c^{\prime} \in \mathfrak{C} \backslash \mathfrak{C}_{0}$. In this case, for any $w \subseteq \mathfrak{C} \backslash \mathfrak{C}_{0}$, there is a natural bijection between $Z\left(D_{u \cup w}\right) \backslash\{a\}$ and $Z\left(D_{v \cup w}\right)$, and we call Khovanov generators $(u \cup w, x)$ and $(v \cup w, y)$ to be a canceling pair if $a$ does not appear in the monomial $x$, and the monomials $x$ and $y$ are related by the above bijection.

(2) The $\operatorname{arc} \alpha_{c}$ has both its endpoints on the same circle in $D_{u}$, and $a \in Z\left(D_{v}\right)$ is one of the two circles obtained by surgering that circle along $\alpha_{c}$, and $a$ is disjoint from $\alpha_{c^{\prime}}$ for all $c^{\prime} \in \mathfrak{C} \backslash \mathfrak{C}_{0}$. In this case, for any $w \subseteq \mathfrak{C} \backslash \mathfrak{C}_{0}$, there is a natural bijection between $Z\left(D_{u \cup w}\right)$ and $Z\left(D_{v \cup w}\right) \backslash\{a\}$, and we call Khovanov generators $(u \cup w, x)$ and $(v \cup w, y)$ to be a canceling pair if $a$ appears in the monomial $y$, and the monomials $x$ and $a^{-1} y$ are related by the above bijection. (This is the dual of the above case.)

Lemma 4.5. Let $\left(\mathfrak{C}_{0}, u, v, c, a\right)$ be a cancellation data. For any $w, w^{\prime} \subseteq \mathfrak{C} \backslash \mathfrak{C}_{0}$, and any Khovanov generators $(u \cup w, x)$ and $\left(v \cup w^{\prime}, y\right)$,

$$
\left\langle\delta_{\text {tot }}((u \cup w, x)),\left(v \cup w^{\prime}, y\right)\right\rangle= \begin{cases}1 & \text { if } w=w^{\prime} \text { and }(u \cup w, x) \text { and }(v \cup w, y) \text { is a canceling pair, } \\ 0 & \text { otherwise. }\end{cases}
$$

In particular, if $S$ is the subset of the Khovanov generators consisting of all the canceling pairs for $\left(\mathfrak{C}_{0}, u, v, c, a\right)$, then the subquotient complex spanned by $S$ is isomorphic to the trivial object in $\mathcal{K}\left(\mathbb{F}_{2}[H, W]\right)$.

Proof. Let us assume the cancellation data $\left(\mathfrak{C}_{0}, u, v, c, a\right)$ corresponds to Case (1) of Definition 4.4. Case (2), being the dual, should follow.

The coefficient $\left\langle\delta_{\text {tot }}((u \cup w, x)),\left(v \cup w^{\prime}, y\right)\right\rangle$ can be non-zero only if $w \subseteq w^{\prime}$, and the resolution configuration $\left(D_{u \cup w}^{v \cup w^{\prime}}, x, y\right)$ either has a non-zero contribution in $\mathfrak{d}$ or a non-zero contribution in $\mathfrak{h}$. To have a non-zero contribution in $\mathfrak{d}, x$ and $y$ must agree on the passive circles, and the active part of $\left(D_{u \cup w}^{v \cup w^{\prime}}, x, y\right)$ must be equivalent to one of the five families described in Definition 2.6. The circle $a$ is one of the active starting circles, has only one arc incident to it, and does not appear in the starting monomial $x$. A quick glance at Figure 2.6 implies the active part of the configuration must be an index- 1 Type-A or Type-E configuration. This occurs when $w=w^{\prime}$, and $(u \cup w, x)$ and $(v \cup w, y)$ form a canceling pair. These are precisely the configurations that contribute to the Khovanov differential $\mathbf{d}_{1}$; see also [Szab, Proof of Theorem 7.2].

In order to have a non-zero contribution in $\mathfrak{h},\left(D_{u \cup w}^{v \cup w^{\prime}}, x, y\right)$ must be equivalent to disjoint union of trees and dual trees, as in Definition 3.2. However, since the arc $\alpha_{c}$ joins the circle $a$ to another circle, and $a$ does not appear in the starting monomial $x$, it cannot be a part of either a tree or a dual tree. Therefore, $\left(D_{u \cup w}^{v \cup w^{\prime}}, x, y\right)$ can never contribute to $\mathfrak{h}$. Consequently, the only contributions come from $\mathfrak{d}$, and as analyzed earlier, they are exactly of the form as described in the lemma.

Now we are almost set to prove invariance under Reidemeister moves. The standard strategy is to delete certain canceling pairs to simplify the chain complex, at the cost of adding new zigzag differentials. For an example of how these zigzag differentials work, assume we have a chain complex freely generated over some ring $R$ with four generators $a_{1}, a_{2}, b_{1}, b_{2}$, and the differential is

$$
\delta a_{1}=b_{1} c_{11}+b_{2} c_{12} \quad \delta a_{2}=b_{1} c_{21}+b_{2} c_{22}
$$




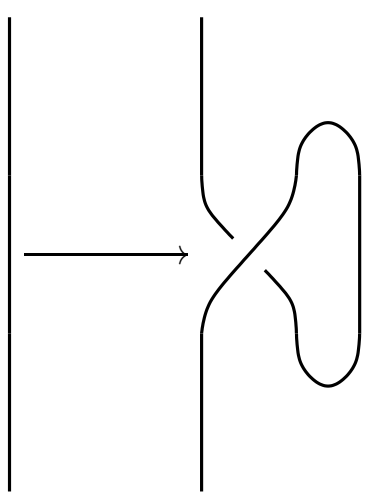

a: RI.

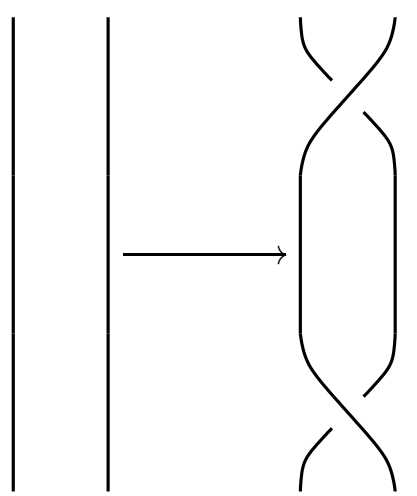

b: RII.

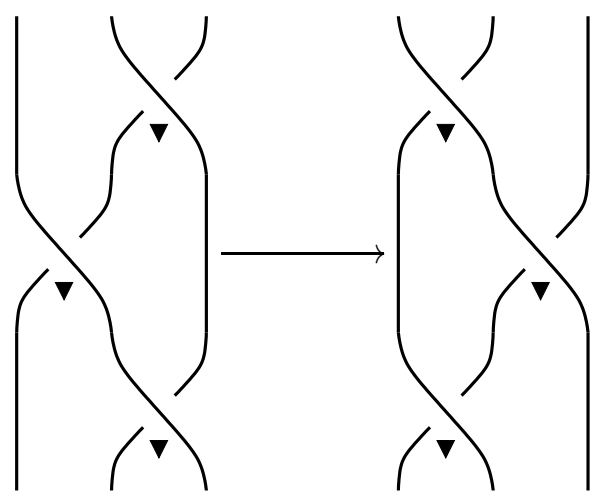

c: RIII.

FiguRE 4.1. The three Reidemeister moves. The orientations of the strands are arbitrary. For RI and RII, the decorations at the new crossings are also arbitrary; for RIII, the decorations are as shown.

and assume $c_{11}$ is a unit. Then we may perform a change of basis

$$
a_{1}^{\prime}=a_{1} \quad a_{2}^{\prime}=a_{2}-a_{1} c_{11}^{-1} c_{21} \quad b_{1}^{\prime}=b_{1}+b_{2} c_{12} c_{11}^{-1} \quad b_{2}^{\prime}=b_{2},
$$

and then the chain complex breaks up into two direct summands, generated by $\left\{a_{1}^{\prime}, b_{1}^{\prime}\right\}$ and $\left\{a_{2}^{\prime}, b_{2}^{\prime}\right\}$. The former is acyclic, so we may cancel it, and then we are left with a complex with just two generators $a_{2}^{\prime}$ and $b_{2}^{\prime}$ with differential $\delta a_{2}^{\prime}=b_{2}^{\prime}\left(c_{22}-c_{12} c_{11}^{-1} c_{21}\right)$. This operation may be viewed as simply canceling the arrow $a_{1} \rightarrow b_{1}$ in the original chain complex and adding an extra zigzag arrow $a_{2} \rightarrow b_{2}$ with coefficient $\left(-c_{12} c_{11}^{-1} c_{21}\right)$.

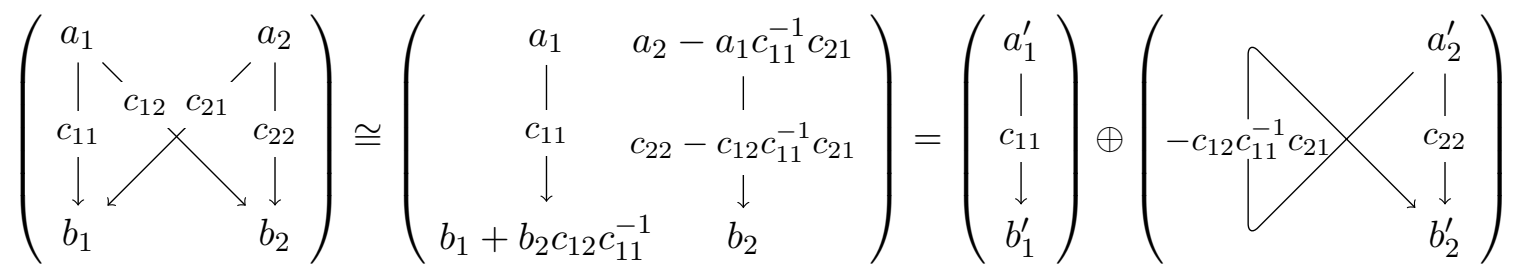

Proposition 4.6. Assume that the decorated pointed link diagram $\left(D^{\prime}, p\right)$ is obtained from the decorated pointed link diagram $(D, p)$ by doing a positive Reidemeister-I stabilization away from the basepoint $p$, see Figure 4.1a, and by extending the decoration arbitrarily on the extra crossing. Then $\left(\mathcal{C}_{\text {tot }}^{-}(D, p)\{1\}, \mathcal{C}_{\text {tot }}(D), \iota(D, p)\right)$ and $\left(\mathcal{C}_{\text {tot }}^{-}\left(D^{\prime}, p\right)\{1\}, \mathcal{C}_{\text {tot }}\left(D^{\prime}\right), \iota\left(D^{\prime}, p\right)\right)$ are isomorphic in $\mathcal{K}_{\mathrm{p}}\left(\mathbb{F}_{2}[H, W]\right)$.

Proof. Let $\mathfrak{C}(D)$ and $\mathfrak{C}\left(D^{\prime}\right)$ be the set of crossings for $D$ and $D^{\prime}$, respectively, and let $\{c\}=$ $\mathfrak{C}\left(D^{\prime}\right) \backslash \mathfrak{C}(D)$. Doing the 0-resolution at $c$ produces a complete circle, say $a$, contained in the neighborhood of $c$ where the Reidemeister-I stabilization takes place. Then $(\{c\}, \varnothing,\{c\}, c, a)$ constitute a cancellation data for $D^{\prime}$ as in Definition 4.4, see also [Kho00, Section 5.1]. 


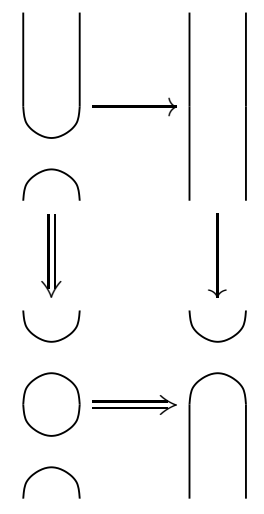

FiguRE 4.2. The partial cube of resolutions of $D^{\prime}$ for the RII invariance. We cancel along the double arrows leaving a subquotient complex isomorphic to the complex for $D$.

Consider the union of all the canceling pairs for this cancellation data. They span a subcomplex of $\mathcal{C}_{\text {tot }}\left(D^{\prime}\right)$, and the corresponding quotient complex is naturally isomorphic to $\mathcal{C}_{\text {tot }}(D)$. Since the subcomplex spanned by the canceling pairs is trivial (from Lemma 4.5), and $p \notin a$, we get $\left(\mathcal{C}_{\text {tot }}^{-}\left(D^{\prime}, p\right)\{1\}, \mathcal{C}_{\text {tot }}\left(D^{\prime}\right), \iota\left(D^{\prime}, p\right)\right)$ is isomorphic to $\left(\mathcal{C}_{\text {tot }}^{-}(D, p)\{1\}, \mathcal{C}_{\text {tot }}(D), \iota(D, p)\right)$ in $\mathcal{K}_{\mathrm{p}}\left(\mathbb{F}_{2}[H, W]\right)$.

Proposition 4.7. Assume that the decorated pointed link diagram $\left(D^{\prime}, p\right)$ is obtained from the decorated pointed link diagram $(D, p)$ by adding a pair of crossings via a Reidemeister-II move away from the basepoint $p$, see Figure 4.1b, and by extending the decoration arbitrarily on the two extra crossings. Then $\left(\mathcal{C}_{\text {tot }}^{-}(D, p)\{1\}, \mathcal{C}_{\text {tot }}(D), \iota(D, p)\right)$ and $\left(\mathcal{C}_{\text {tot }}^{-}\left(D^{\prime}, p\right)\{1\}, \mathcal{C}_{\text {tot }}\left(D^{\prime}\right), \iota\left(D^{\prime}, p\right)\right)$ are isomorphic in $\mathcal{K}_{\mathrm{p}}\left(\mathbb{F}_{2}[H, W]\right)$.

Proof. The argument is similar to the previous one. Let $\left\{c_{1}, c_{2}\right\}=\mathfrak{C}\left(D^{\prime}\right) \backslash \mathfrak{C}(D)$, numbered top to bottom (as per Figure 4.1b). Then the subquotient complex of $\mathcal{C}_{\text {tot }}\left(D^{\prime}\right)$ spanned by the Khovanov generators that live over the 0 -resolution at $c_{1}$ and the 1-resolution at $c_{2}$ is naturally isomorphic (after the correct bi-grading shifts) to $\mathcal{C}_{\text {tot }}(D)$.

Doing the 1-resolution at $c_{1}$ and the 0 -resolution at $c_{2}$ produces a complete circle, say $a$, contained in the neighborhood of $\left\{c_{1}, c_{2}\right\}$ where the Reidemeister-II move takes place. Then both $\left(\left\{c_{1}, c_{2}\right\}, \varnothing,\left\{c_{1}\right\}, c_{1}, a\right)$ and $\left(\left\{c_{1}, c_{2}\right\},\left\{c_{1}\right\},\left\{c_{1}, c_{2}\right\}, c_{2}, a\right)$ are cancellation data for $D^{\prime}$, see also [Kho00, Section 5.3].

Using Lemma 4.5, we can first cancel the subcomplex of $\mathcal{C}_{\text {tot }}\left(D^{\prime}\right)$ spanned by all the canceling pairs for $\left(\left\{c_{1}, c_{2}\right\},\left\{c_{1}\right\},\left\{c_{1}, c_{2}\right\}, c_{2}, a\right)$, and then cancel the quotient complex spanned by the canceling pairs of $\left(\left\{c_{1}, c_{2}\right\}, \varnothing,\left\{c_{1}\right\}, c_{1}, a\right)$. After all the cancellations, we will be left with the subquotient complex isomorphic to $\mathcal{C}_{\text {tot }}(D)$, and (and since $p \notin a$ ) this establishes the isomorphism between $\left(\mathcal{C}_{\text {tot }}^{-}(D, p)\{1\}, \mathcal{C}_{\text {tot }}(D), \iota(D, p)\right)$ and $\left(\mathcal{C}_{\text {tot }}^{-}\left(D^{\prime}, p\right)\{1\}, \mathcal{C}_{\text {tot }}\left(D^{\prime}\right), \iota\left(D^{\prime}, p\right)\right)$ in $\mathcal{K}_{\mathrm{p}}\left(\mathbb{F}_{2}[H, W]\right)$. See also Figure 4.2. 


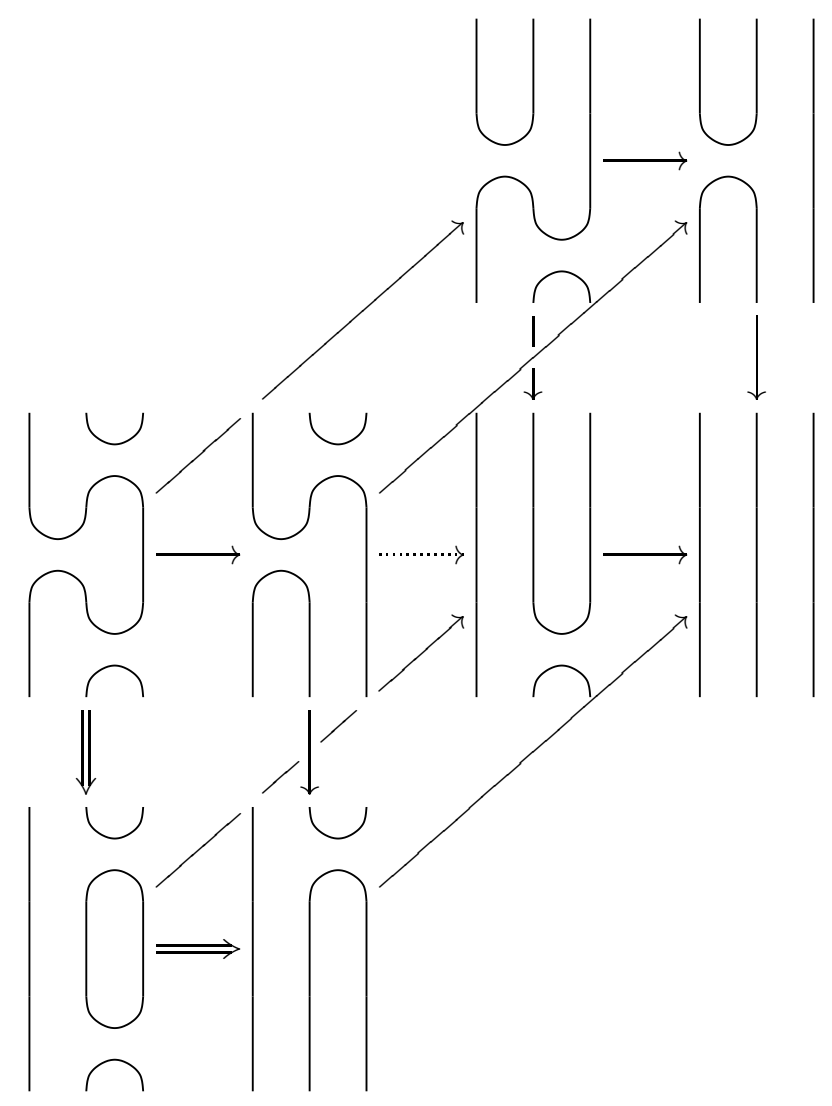

Figure 4.3. The partial cube of resolutions of $D$ for the RIII invariance. We cancel along the double arrows; the remainder is not a subquotient complex, so the cancellation produces the new dotted arrow. We can produce similar cancellations for $D^{\prime}$ to arrive at the same diagram.

Proposition 4.8. Assume that the decorated pointed link diagram $\left(D^{\prime}, p\right)$ is obtained from the decorated pointed link diagram $(D, p)$ by performing a Reidemeister-III move aways from the basepoint $p$, with the decorations being consistent, as shown in Figure 4.1c. Then $\left(\mathcal{C}_{\text {tot }}^{-}(D, p)\{1\}, \mathcal{C}_{\text {tot }}(D)\right.$, $\iota(D, p))$ and $\left(\mathcal{C}_{\text {tot }}^{-}\left(D^{\prime}, p\right)\{1\}, \mathcal{C}_{\text {tot }}\left(D^{\prime}\right), \iota\left(D^{\prime}, p\right)\right)$ are isomorphic in $\mathcal{K}_{\mathrm{p}}\left(\mathbb{F}_{2}[H, W]\right)$.

Proof. The proof is slightly different from the previous ones. We will do cancellations as before, but on each of the diagrams $D$ and $D^{\prime}$, and reduce both to the same complex. Let us describe the cancellations for $D$ in more detail. 
Let $c_{1}, c_{2}, c_{3}$ be the crossings of $D$ where the Reidemeister-III move takes place, numbered top to bottom (as per Figure 4.1c). Doing the 0 -resolutions at $c_{1}$ and $c_{3}$, and the 1-resolution at $c_{2}$ produces a complete circle, say $a$, contained in the neighborhood of $\left\{c_{1}, c_{2}, c_{3}\right\}$ where the ReidemeisterIII move takes place. Then all three of $\left(\left\{c_{1}, c_{2}, c_{3}\right\}, \varnothing,\left\{c_{2}\right\}, c_{2}, a\right),\left(\left\{c_{1}, c_{2}, c_{3}\right\},\left\{c_{2}\right\},\left\{c_{1}, c_{2}\right\}, c_{1}, a\right)$, and $\left(\left\{c_{1}, c_{2}, c_{3}\right\},\left\{c_{2}\right\},\left\{c_{2}, c_{3}\right\}, c_{3}, a\right)$ are cancellation data for $D$, see also [Kho00, Section 5.5] and [Szab, Theorem 7.2].

Using Lemma 4.5, we first cancel the quotient complex of $\mathcal{C}_{\text {tot }}(D)$ spanned by all the canceling pairs for $\left(\left\{c_{1}, c_{2}, c_{3}\right\}, \varnothing,\left\{c_{2}\right\}, c_{2}, a\right)$. We then cancel all the canceling pairs for $\left(\left\{c_{1}, c_{2}, c_{3}\right\},\left\{c_{2}\right\}\right.$, $\left.\left\{c_{2}, c_{3}\right\}, c_{3}, a\right)$; this is neither a subcomplex nor a quotient complex, so this cancellation produces new zigzag differentials, as in Equation (4.1). The new differentials go from the Khovanov generators living over $\left\{c_{3}\right\} \cup w_{1}$ to Khovanov generators living over $\left\{c_{1}, c_{2}\right\} \cup w_{2}$ as $w_{1}, w_{2}$ vary over subsets of $\mathfrak{C}(D) \backslash\left\{c_{1}, c_{2}, c_{3}\right\}$. This is shown by the dotted arrow in Figure 4.3.

Let us analyze the new differentials in more detail. First set up some more notation. Let $C_{2}^{+}$, $C_{3}, C_{12}$, and $C_{23}$ denote the subquotient complexes of $\mathcal{C}_{\text {tot }}(D)$ spanned by Khovanov generators living over $\left\{c_{2}\right\} \cup w$ with $a$ not appearing in the monomial, $\left\{c_{3}\right\} \cup w,\left\{c_{1}, c_{2}\right\} \cup w,\left\{c_{2}, c_{3}\right\} \cup w$, respectively, for arbitrary $w \subseteq \mathfrak{C}(D) \backslash\left\{c_{1}, c_{2}, c_{3}\right\}$. There is a natural identification $C_{12} \cong C_{23}$ since the resolutions $D_{\left\{c_{1}, c_{2}\right\} \cup w}$ and $D_{\left\{c_{2}, c_{3}\right\} \cup w}$ are identical outside a neighborhood of $a$, and are canonically isotopic to each other inside the neighborhood. The new differential is from $C_{3}$ to $C_{12}$, and we claim that it is identical (under the above identification) to the part of the old differential $\delta_{\text {tot }}$ that went from $C_{3}$ to $C_{23}$. To see this, recall that both $\left(\left\{c_{1}, c_{2}, c_{3}\right\},\left\{c_{2}\right\},\left\{c_{1}, c_{2}\right\}, c_{1}, a\right)$, and $\left(\left\{c_{1}, c_{2}, c_{3}\right\},\left\{c_{2}\right\},\left\{c_{2}, c_{3}\right\}, c_{3}, a\right)$ are cancellation data for $D$. Therefore, using Lemma 4.5, only $\mathbf{d}_{1}$ contributes the part of the differential $\delta_{\text {tot }}$ that goes from $C_{2}^{+}$to $C_{12}$ or from $C_{2}^{+}$to $C_{23}$; and in either case, it produces a bijection between the Khovanov generators in $C_{2}^{+}$with the Khovanov generators in $C_{12}$ or $C_{23}$; the induced bijection between the Khovanov generators in $C_{12}$ and $C_{23}$ is easily seen to be the above identification. The new zigzag differential from $C_{3}$ to $C_{12}$ is obtained by composing the part of the old differential $\delta_{\text {tot }}$ from $C_{3}$ to $C_{23}$, and then mapping $C_{23}$ to $C_{12}$ by the above bijection. This shows that the new differential is identical to the part of the old differential $\delta_{\text {tot }}$ that went from $C_{3}$ to $C_{23}$.

We can perform similar cancellations for the diagram $D^{\prime}$. To wit, if $c_{1}^{\prime}, c_{2}^{\prime}, c_{3}^{\prime}$ are the crossings of $D^{\prime}$ where the Reidemeister-III move takes place, numbered top to bottom (as per Figure 4.1c), then doing the 0-resolutions at $c_{1}^{\prime}$ and $c_{3}^{\prime}$ and the 1-resolution at $c_{2}^{\prime}$ produces a complete circle, say $a^{\prime}$, contained in the neighborhood where the Reidemeister-III move takes place. Then we cancel all Khovanov generators for the cancellation data $\left(\left\{c_{1}^{\prime}, c_{2}^{\prime}, c_{3}^{\prime}\right\}, \varnothing,\left\{c_{2}^{\prime}\right\}, c_{2}^{\prime}, a^{\prime}\right)$ and $\left(\left\{c_{1}^{\prime}, c_{2}^{\prime}, c_{3}^{\prime}\right\},\left\{c_{2}^{\prime}\right\}\right.$, $\left.\left\{c_{2}^{\prime}, c_{3}^{\prime}\right\}, c_{3}^{\prime}, a^{\prime}\right)$. Since we had decorated the diagrams $D$ and $D^{\prime}$ coherently, it is straightforward to see that after performing these cancellations, we end up in an isomorphic picture. (The postcancellation complexes for $D$ and $D^{\prime}$ are identical outside the region where the Reidemeister-III move occurs, and inside the region, the corresponding resolutions are canonically isotopic to one another.)

This establishes (after noting that the basepoint $p$ is not in the circle $a$ ) that $\left(\mathcal{C}_{\text {tot }}^{-}(D, p)\{1\}\right.$, $\left.\mathcal{C}_{\text {tot }}(D), \iota(D, p)\right)$ and $\left(\mathcal{C}_{\text {tot }}^{-}\left(D^{\prime}, p\right)\{1\}, \mathcal{C}_{\text {tot }}\left(D^{\prime}\right), \iota\left(D^{\prime}, p\right)\right)$ are isomorphic in $\mathcal{K}_{\mathrm{p}}\left(\mathbb{F}_{2}[H, W]\right)$.

Lemma 4.9. If $(D, p)$ and $\left(D^{\prime}, p^{\prime}\right)$ are two decorated pointed link diagrams representing isotopic pointed links, then they can be connected by some sequence of positive Reidemeister-I moves, 

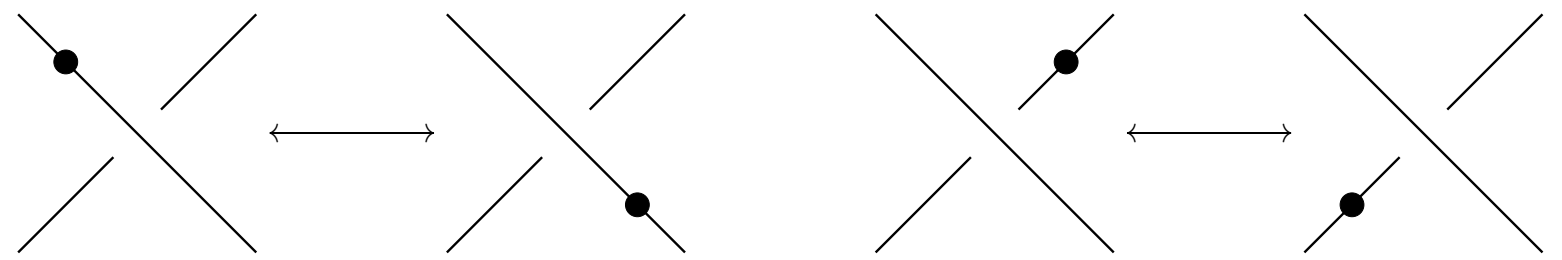

Figure 4.4. Additional moves for the basepoint.
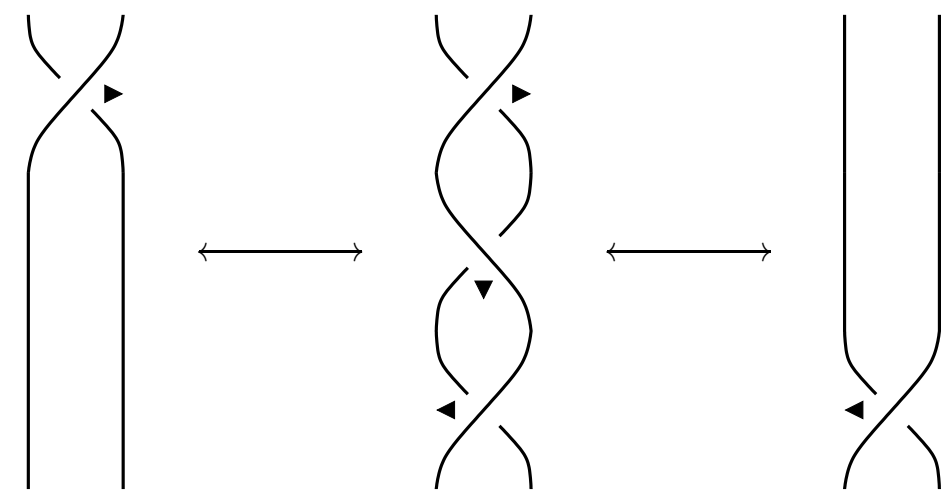

FiguRE 4.5. Changing the decoration at a single crossing.

Reidemeister-II moves, and Reidemeister-III moves, as described in the statements of Propositions 4.6-4.8 and Figure 4.1, their inverses, and isotopy in $S^{2}$.

Proof. This is essentially Reidemeister's theorem which states that any two link diagrams for the same link can be connected by isotopy in $\mathbb{R}^{2}$ and the three Reidemeister moves (and in particular, we only need one variant for each of the Reidemeister I and III moves).

In presence of a single basepoint, we need two additional moves as shown in Figure 4.4, corresponding to moving the basepoint past a strand. However, as observed in [Kho03, Section 3], these moves may be achieved via the usual three Reidemeister moves away from the basepoint and isotopy in $S^{2}$.

Finally, with regard to decorations, we need an additional move which changes the decoration at a single crossing. This move may be achieved by two Reidemeister II moves, as shown in Figure 4.5.

Proof of Proposition 4.2. This is immediate from Lemma 4.9 and Propositions 4.6-4.8. The only thing to note is that the contribution functions $\mathfrak{d}$ and $\mathfrak{h}$ satisfy the naturality rule, i.e., they are preserved under isotopy in $S^{2}$; consequently, the chain complex $\mathcal{C}_{\text {tot }}$ defined using $\mathfrak{d}$ and $\mathfrak{h}$ also remains invariant under isotopy in $S^{2}$. 


\section{Properties}

From this section onwards, we will restrict to the unpointed case, and only study the unreduced version $\mathcal{C}_{\text {tot }}$. In Propositions 4.6-4.8, we associated isomorphisms in $\mathcal{K}\left(\mathbb{F}_{2}[H, W]\right)$ corresponding to knot isotopy. We will now construct morphisms in $\mathcal{K}\left(\mathbb{F}_{2}[H, W]\right)$ for three additional local moves.

Definition 5.1. Fix a decorated link diagram $D$, and let $D^{\prime}$ be the decorated link diagram obtained from $D$ by adding a small unknotted circle $a$ disjoint from the $D$. (The transformation $D \rightarrow D^{\prime}$ is usually called a 'birth', and it corresponds to a link cobordism in $\mathbb{R}^{3} \times[0,1]$ with a single index-zero critical point. The transformation $D^{\prime} \rightarrow D$ is usually called a 'death', and it corresponds to a link cobordism in $\mathbb{R}^{3} \times[0,1]$ with a single index-two critical point.) We have a decomposition

$$
\mathcal{C}_{\text {tot }}\left(D^{\prime}\right) \cong \mathcal{C}_{\text {tot }}\left(D^{\prime}\right)_{0} \oplus \mathcal{C}_{\text {tot }}\left(D^{\prime}\right)_{1}
$$

where $\mathcal{C}_{\text {tot }}\left(D^{\prime}\right)_{0}$ is the direct summand of $\mathcal{C}_{\text {tot }}\left(D^{\prime}\right)$ where the circle $a$ does not appear in the monomials for the Khovanov generators, while $\mathcal{C}_{\text {tot }}\left(D^{\prime}\right)_{1}$ is the direct summand of $\mathcal{C}_{\text {tot }}\left(D^{\prime}\right)$ where the circle $a$ does appear in the monomials. Each of $\mathcal{C}_{\text {tot }}\left(D^{\prime}\right)_{0}$ and $\mathcal{C}_{\text {tot }}\left(D^{\prime}\right)_{1}$ is identified with $\mathcal{C}_{\text {tot }}(D)$, after shifting the quantum gradings correctly.

To a birth, we associate a morphism from $\mathcal{C}_{\text {tot }}(D)$ to $\mathcal{C}_{\text {tot }}\left(D^{\prime}\right)\{1\}$ in $\mathcal{K}\left(\mathbb{F}_{2}[H, W]\right)$ as the composition

$$
\mathcal{C}_{\text {tot }}(D) \cong \mathcal{C}_{\text {tot }}\left(D^{\prime}\right)_{0}\{1\} \hookrightarrow \mathcal{C}_{\text {tot }}\left(D^{\prime}\right)_{0}\{1\} \oplus \mathcal{C}_{\text {tot }}\left(D^{\prime}\right)_{1}\{1\} \cong \mathcal{C}_{\text {tot }}\left(D^{\prime}\right)\{1\},
$$

where the inclusion is an inclusion as a direct summand.

To a death, we associate a morphism from $\mathcal{C}_{\text {tot }}\left(D^{\prime}\right)$ to $\mathcal{C}_{\text {tot }}(D)\{1\}$ in $\mathcal{K}\left(\mathbb{F}_{2}[H, W]\right)$ as the composition

$$
\mathcal{C}_{\text {tot }}\left(D^{\prime}\right) \cong \mathcal{C}_{\text {tot }}\left(D^{\prime}\right)_{0} \oplus \mathcal{C}_{\text {tot }}\left(D^{\prime}\right)_{1} \rightarrow \mathcal{C}_{\text {tot }}\left(D^{\prime}\right)_{1} \cong \mathcal{C}_{\text {tot }}(D)\{1\},
$$

where the surjection is a projection to a direct summand.

Definition 5.2. Assume two decorated link diagrams $D_{0}$ and $D_{1}$ are related as shown in Figure 5.1. That is, assume that there is a decorated link diagram $D$ with a distinguished $\operatorname{crossing} c$, so that resolving $c$ by the $i$-resolution produces $D_{i}$, for $i=0,1$; and further assume the link diagrams $D_{0}$ and $D_{1}$ can be, and are, oriented coherently. (The transformation $D_{0} \rightarrow D_{1}$ is usually called a 'saddle', and it corresponds to a link cobordism in $\mathbb{R}^{3} \times[0,1]$ with a single index-one critical point.) After an overall shift of the bigradings (which may depend on the diagrams), there is an identification

$$
\mathcal{C}_{\text {tot }}(D) \cong \operatorname{Cone}\left(f: \mathcal{C}_{\text {tot }}\left(D_{0}\right) \rightarrow \mathcal{C}_{\text {tot }}\left(D_{1}\right)\{-1\}\right) .
$$

where $f$ is the part of the differential $\delta_{\text {tot }}$ for $\mathcal{C}_{\text {tot }}(D)$ that goes from the 0-resolution at $c$ to the 1-resolution at $c$. To the saddle move $D_{0} \rightarrow D_{1}$, we associate the morphism $f$ from $\mathcal{C}_{\text {tot }}\left(D_{0}\right)$ to $\mathcal{C}_{\text {tot }}\left(D_{1}\right)\{-1\}$ in $\mathcal{K}\left(\mathbb{F}_{2}[H, W]\right)$.

To a link cobordism in $\mathbb{R}^{3} \times[0,1]$, one can associate maps on Khovanov chain complex [Kho00, Jac04, Kho06, CMW09] and on the Bar-Natan chain complex [BN05, CMW09]. These maps are defined by composing maps associated to elementary moves, namely the three Reidemeister moves, birth, death, and saddle. We may also use our maps from Propositions 4.6-4.8 and Definitions 5.15.2 to define map associated to link cobordisms presented as a sequence of elementary moves. We will not prove that this map is well-defined in $\mathcal{K}\left(\mathbb{F}_{2}[H, W]\right)$, that is, it only depends on the isotopy 


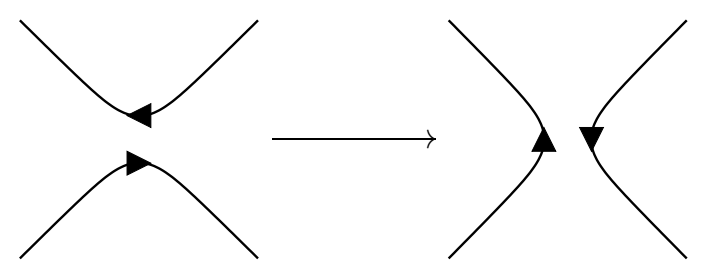

FiguRE 5.1. The saddle cobordism from $D_{0}$ to $D_{1}$. Note that the link diagrams are oriented coherently.

class of the link cobordism, and not on a choice of presentation as a sequence of elementary moves. Nevertheless, when we specialize $W=0$, we get the existing link cobordism map on the Bar-Natan theory.

Proposition 5.3. For any link cobordism in $\mathbb{R}^{3} \times[0,1]$ viewed as a sequence of elementary moves, the maps on $\mathcal{C}_{\text {tot }}$ defined in Propositions 4.6-4.8 and Definitions 5.1-5.2 induce the standard link cobordism maps in the Bar-Natan theory $\mathcal{C}_{B N}$.

Proof. This is immediate from the definitions (which we have not given here) of the Bar-Natan link cobordism maps, which are specializations [BN05, Section 9.3] of maps defined in a more general setting [BN05, Sections 4.3 and 8.1].

Next, we will use these link cobordism maps to prove some structure theorems for the total homology. Before proceeding, let us collect a few facts about the Bar-Natan theory.

$(\mathrm{BN}-1)$ For an $l$-component link $L$, the homology of the localized Bar-Natan complex $\{H\}^{-1} \mathcal{C}_{B N}$ is $2^{l}$ copies of $\mathbb{F}_{2}\left[H, H^{-1}\right]$, while the homology of the filtered Bar-Natan complex $\mathcal{C}_{f B N}=$ $\mathcal{C}_{\text {tot }} /\{H=1, W=0\}$ has rank $2^{l}$ [Lee05, Tur06]; in either case, the generators correspond to the orientations of $L$, and the $\operatorname{gr}_{h}$-preserving reduction $\{H\}^{-1} \mathcal{C}_{B N} \rightarrow \mathcal{C}_{f B N}$ preserves this correspondence. (Since $\mathcal{C}_{f B N} \otimes \mathbb{F}_{2}[\mathbb{Z}]$ can be identified with $\{H\}^{-1} \mathcal{C}_{B N}$, as in the proof of Proposition 5.4, the two statements are equivalent.) In more detail, consider some orientation $o$ on the link $L$, presented as a link diagram $D$ in the plane $\mathbb{R}^{2}$. Fix a checkerboard coloring of the complement of $D$ in $\mathbb{R}^{2}$; for concreteness, one usually decrees the unbounded region to be colored white. Consider the oriented resolution of $D$ according to the orientation $o$, and let $\left\{x_{1}, \ldots, x_{k}\right\}$ be the complete circles of the resolution. Then each of the individual circles $\left\{x_{i}\right\}$ are also oriented according to $o$. Consider the one-variable polynomial (over $\mathbb{F}_{2}[H]$ ) in $x_{i}$, which is $x_{i}$ or $H+x_{i}$, depending on whether $x_{i}$ is oriented as the boundary of a black region or a white region, respectively; then consider the product of all these $k$ one-variable polynomials. This $k$-variable polynomial may be viewed as a linear combination over $\mathbb{F}_{2}[H]$ of square-free monomials in the circles $\left\{x_{i}\right\}$, and thereby viewed as a linear combination of the Khovanov generators over this oriented resolution, see also (X-3) from Section 2. This linear combination represents the generator corresponding to $o$ in $\{H\}^{-1} \mathcal{C}_{B N}$. Let $g_{B N}(o)$ be the corresponding generator in $H_{*}\left(\mathcal{C}_{f B N}\right)$. 
In particular, note that the homological grading of $g_{B N}(o)$ is given by the linking number

$$
\operatorname{gr}_{h}\left(g_{B N}(o)\right)=2 \operatorname{lk}\left(L_{0}, L \backslash L_{0}\right)
$$

where $L_{0} \subseteq L$ is the sublink where the orientation $o$ agrees with the starting orientation of $L$ (the one that was used to define the homological grading $\mathrm{gr}_{h}$ in the first place, cf. (X-4) from Section 2), with the following understanding: the linking number is computing after orienting both $L_{0}$ and $L \backslash L_{0}$ according to the starting orientation of $L$; and the linking number with the empty link is zero.

(BN-2) For any oriented link cobordism from $L_{1}$ to $L_{2}$ in $\mathbb{R}^{3} \times[0,1]$ without any closed components, and for any orientation $o$ on $L_{1}$, the Bar-Natan link cobordism map on $\mathcal{C}_{f B N}$ acts as follows on the generator $g_{B N}(o)$,

$$
g_{B N}(o) \mapsto \sum_{\substack{o^{\prime} \text { orientation on } L_{2} \\ o \text { and } o^{\prime} \text { extend to an orientation on the cobordism }}} g_{B N}\left(o^{\prime}\right),
$$

see [Ras10], see also [LS14].

$(\mathrm{BN}-3)$ For a knot $K$, the Rasmussen $s$-invariant of the knot (over $\mathbb{F}_{2}$ ) is defined as

$$
\begin{aligned}
s(K) & =\max \left\{n \mid \mathcal{F}_{n} \mathcal{C}_{B N}(K) \text { contains a representative for } g_{B N}(o)\right\}+1 \\
& =\max \left\{n \mid \mathcal{F}_{n} \mathcal{C}_{B N}(K) \text { contains a representative for } g_{B N}(-o)\right\}+1 \\
& =\max \left\{n \mid \mathcal{F}_{n} \mathcal{C}_{B N}(K) \text { contains a representative for } g_{B N}(o)+g_{B N}(-o)\right\}-1,
\end{aligned}
$$

where $o$ is any orientation on $K$ and $\mathcal{F}_{n} \mathcal{C}_{B N}(K)$ is the subcomplex of $\mathcal{C}_{B N}(K)$ supported in quantum grading $n$ or more. This was originally defined over any field of characteristic different from 2 in [Ras10], and extended to $\mathbb{F}_{2}$ in [LS14].

Proposition 5.4. Fix any l-component link L. The following hold:

$(R-1)$ The homology of $\mathcal{C}_{\text {fHtot }}=\mathcal{C}_{\text {tot }} /\{H=1\}$ is isomorphic to $2^{l}$ copies of $\mathbb{F}_{2}[W]$.

$\left(R\right.$-2) The homology of $\{H\}^{-1} \mathcal{C}_{\text {tot }}$ is isomorphic to $2^{l}$ copies of $\mathbb{F}_{2}\left[H, H^{-1}, W\right]$

$(R-3)$ The homology of $\mathcal{C}_{\text {ftot }}=(\mathcal{C}, \mathbf{d}+\mathbf{h})$ has rank $2^{l}$.

In each case, the $2^{l}$ generators are in a canonical correspondence with the orientations of $L$ (as was the case for $\{H\}^{-1} \mathcal{C}_{B N}$ and $\left.\mathcal{C}_{f B N}, c f .(B N-1)\right)$, and the reductions

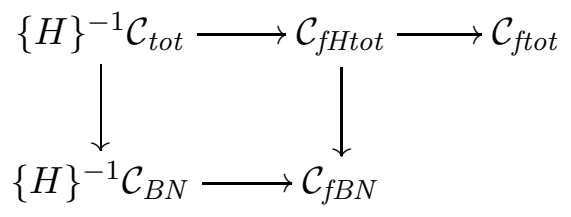

preserve this correspondence, and the left four reductions (the ones forming the square) also preserve the homological grading.

Towards this end, we will need to understand the spectral sequence $H_{*}\left(\mathcal{C}_{f H t o t} /\{W=0\}\right) \otimes$ $\mathbb{F}_{2}[W] \rightrightarrows H_{*}\left(\mathcal{C}_{f H t o t}\right)$. The chain complex $\mathcal{C}_{\text {fHtot }}$ is singly graded by $\mathrm{gr}_{h}$, and carries a filtration by powers of $W$. Its associated graded object is isomorphic to

$$
\mathcal{C}_{\text {fHtot }} /\{W=0\} \otimes \mathbb{F}_{2}[W]=\mathcal{C}_{f B N} \otimes \mathbb{F}_{2}[W],
$$


whose homology is $2^{l}$ copies of $\mathbb{F}_{2}[W]$, via $(\mathrm{BN}-1)$. The filtrations induce a spectral sequence over $\mathbb{F}_{2}[W]$ (see for example [McC00, Theorem 2.6]) starting at the homology of the associated graded object, and converging to the homology of $\mathcal{C}_{f H t o t}$. Since the complex $\mathcal{C}_{f H t o t}$ is finitely generated over $\mathbb{F}_{2}[W]$, the spectral sequence is forced to collapse after finitely many pages. We will in fact show that the spectral sequence collapses immediately.

Lemma 5.5. The above spectral sequence $H_{*}\left(\mathcal{C}_{f B N}\right) \otimes \mathbb{F}_{2}[W] \rightrightarrows H_{*}\left(\mathcal{C}_{\text {fHtot }}\right)$ has no higher differentials.

Proof. We first prove this when the link $L$ is a disjoint union of some copies of the Hopf link and some copies of the unknot. Since adding a disjoint unknot component has the effect of tensoring everything with a two-dimensional vector space, we might assume $L$ is merely a disjoint union of Hopf link components, and let $M_{1}, \ldots, M_{k}$ be the components (so that $L$ has $l=2 k$ link components). Fix a link diagram for $L$ with $2 k$ crossings. Let $c_{i 1}$ and $c_{i 2}$ be the two crossings in the diagram for $M_{i}$, and fix a decoration on the link diagram so that the surgery arcs $\alpha_{c_{i 1}}$ and $\alpha_{c_{i 2}}$ are oriented in parallel.

We will first analyze the complex $\mathcal{C}_{\text {fHtot }}\left(M_{i}\right)$. For any subset $u \subseteq\left\{c_{i 1}, c_{i 2}\right\}$, let $\bar{u} \in\{0,1\}^{2}$ be the corresponding vertex (that is, $c_{i j} \in u$ iff $\bar{u}_{j}=1$ ). Let $\left\{x_{j}^{\bar{u}}\right\}$ be the circles appearing in the complete resolution of $M_{i}$ corresponding to $u$; therefore, the chain group over $u$ will be generated by the square-free monomials in $x_{j}^{\bar{u}}$; for clarity, we will denote the monomial 1 as $1^{\bar{u}}$. Then the complex $\mathcal{C}_{\text {fHtot }}\left(M_{i}\right)$ is the following:

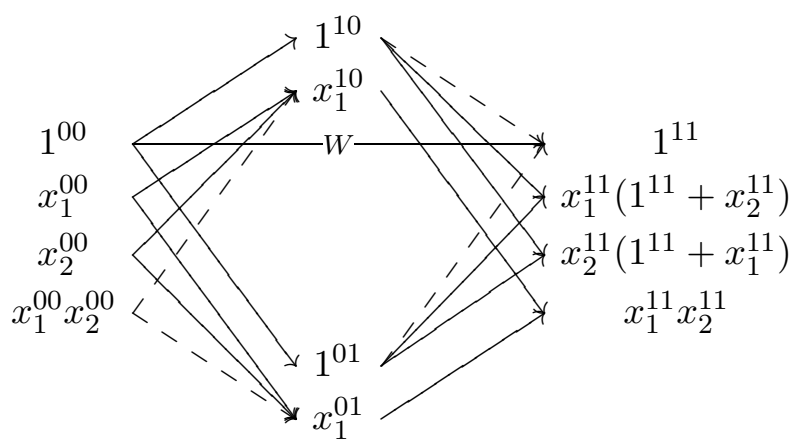

Here, we have performed a change of basis on the generators coming from the resolution at $\left\{c_{i 1}, c_{i 2}\right\}$. The dotted arrows come from the differentials $\mathbf{h}_{1}$. The short solid arrows come from the differentials $\mathbf{d}_{1}$, while the long solid arrow (from $1^{00}$ to $1^{11}$ ) comes from the differential $\mathbf{d}_{2}$; it picks up a power of $W$, which we have indicated.

We observe that after this change of basis, the complex $\mathcal{C}_{\text {fHtot }}\left(M_{i}\right)$ breaks up into two direct summands: the summand $S_{i}$ generated by $1^{00}, 1^{10}, 1^{01}, 1^{11}, x_{1}^{11}\left(1^{11}+x_{2}^{11}\right)$, and $x_{2}^{11}\left(1^{11}+x_{1}^{11}\right)$; and the summand $T_{i}$ generated by $x_{1}^{00}, x_{2}^{00}, x_{1}^{00} x_{2}^{00}, x_{1}^{10}, x_{1}^{01}$, and $x_{1}^{11} x_{2}^{11}$. Furthermore, $S_{i}$ contains two of the four homology generators of the filtered Bar-Natan complex $\mathcal{C}_{f B N}$, namely, $x_{1}^{11}\left(1^{11}+x_{2}^{11}\right)$ and $x_{2}^{11}\left(1^{11}+x_{1}^{11}\right)$, and they live in the same homological grading; and $T_{i}$ contains the other two homology generators of $\mathcal{C}_{f B N}$, namely, $x_{1}^{00}+x_{1}^{00} x_{2}^{00}$ and $x_{2}^{00}+x_{1}^{00} x_{2}^{00}$, and they too live in the same homological grading.

Now look at the complex for $L=\coprod_{i} M_{i}$. The chain complex $\mathcal{C}_{f H t o t}(L)$ is not directly related to the tensor product of the chain complexes $\mathcal{C}_{\text {fHtot }}\left(M_{i}\right)$. The chain group is indeed the tensor 


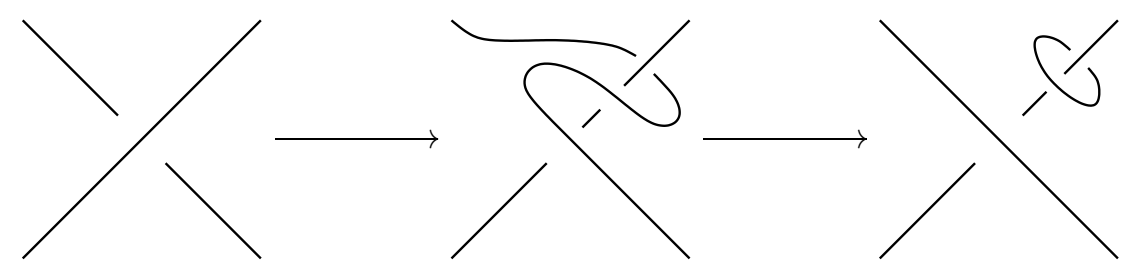

FiguRE 5.2. Performing a crossing change by a saddle (which splits a component into two) at the cost of introducing a meridional circle on one of the strands.

product of the individual chain groups, and the differential coming from $\mathbf{d}$ behaves like a tensor product, but the differential coming from $\mathbf{h}$ is gotten by applying it to any non-empty subset of the individual chain groups (as opposed to just one, which would have been the case for the tensor product). Since the only non-zero terms in the differential for $\mathcal{C}_{f H t o t}\left(M_{i}\right)$ come from $\mathbf{d}_{1}, \mathbf{d}_{2}$ and $\mathbf{h}_{1}$, we can write the differential for $\mathcal{C}_{\text {fHtot }}(L)$ succinctly as follows. For any generators $\gamma_{i} \in \mathcal{C}\left(M_{i}\right)$, the differential on $\gamma_{1} \otimes \cdots \otimes \gamma_{k}$ in $\mathcal{C}_{\text {fHtot }}(L)$ is the following sum:

$$
\begin{gathered}
\sum_{i} \gamma_{1} \otimes \cdots \otimes\left(\mathbf{d}_{1}+W \mathbf{d}_{2}\right)\left(\gamma_{i}\right) \otimes \cdots \otimes \gamma_{k}+\sum_{\varnothing \neq A \subseteq\{1, \ldots, k\}} W^{|A|-1}\left(\bigotimes_{i \in A} \mathbf{h}_{1}\left(\gamma_{i}\right)\right) \otimes\left(\bigotimes_{i \notin A} \gamma_{i}\right) \\
=\sum_{i} \gamma_{1} \otimes \cdots \otimes\left(\mathbf{d}_{1}+W \mathbf{d}_{2}\right)\left(\gamma_{i}\right) \otimes \cdots \otimes \gamma_{k} \\
+\frac{\left(\operatorname{Id}+W \mathbf{h}_{1}\right)\left(\gamma_{1}\right) \otimes \cdots \otimes\left(\operatorname{Id}+W \mathbf{h}_{1}\right)\left(\gamma_{k}\right)-\gamma_{1} \otimes \cdots \otimes \gamma_{k}}{W} .
\end{gathered}
$$

Therefore, despite not being the tensor product, we still get $2^{k}$ direct summands for $\mathcal{C}_{\text {fHtot }}(L)$ coming from the direct summands $S_{i}$ and $T_{i}$ for $\mathcal{C}_{f H t o t}\left(M_{i}\right)$, for $i=1, \ldots, k$. That is, the chain complex $\mathcal{C}_{\text {fHtot }}(L)$ can be viewed as $2^{k}$ different filtered chain complexes, not interacting with one another, each with an associated spectral sequence. Furthermore, each summand contains $2^{k}$ homology generators of the filtered Bar-Natan complex $\mathcal{C}_{f B N}$, all living in the same homological grading.

We have so far not delved into the details of the the spectral sequence associated to a filtered chain complex. Peeking into [McC00, the proof of Theorem 2.6], we see that the higher differentials correspond to zigzags of the same form as described in Equation (4.1). Therefore, the spectral sequence associated to a direct sum of filtered chain complexes is the direct sum of the individual spectral sequences. Consequently, in the situation at hand, the entire spectral sequence decomposes into $2^{k}$ summands. On the other hand, it is easy to see from grading considerations that the higher differentials are zero for each of the summands. To wit, the homology of the first page of each summand has $2^{k}$ generators (over $\mathbb{F}_{2}[W]$ ) living in the same homological grading. Since the higher differentials increase homological grading $\operatorname{gr}_{h}$ by one, and $\operatorname{gr}_{h}(W)=-1$, we see that there are no higher differentials.

This completes the proof that the spectral sequence $H_{*}\left(\mathcal{C}_{f B N}(L)\right) \otimes \mathbb{F}_{2}[W] \rightrightarrows H_{*}\left(\mathcal{C}_{\text {fHtot }}(L)\right)$ has no higher differentials when $L$ is a disjoint union of some copies of the Hopf link and some copies 
of the unknot. To see how this implies the general statement, we will construct an oriented link cobordism $S$ in $\mathbb{R}^{3} \times[0,1]$ from our starting link $L$ to some other link $L^{\prime}$ which is a disjoint union of some copies of the Hopf link and some copies of the unknot, satisfying the following properties:

(1) The map $\pi_{0}(L) \rightarrow \pi_{0}(S)$ (induced from the inclusion $\left.L \cong S \cap\left(\mathbb{R}^{3} \times\{0\}\right) \hookrightarrow S\right)$ is a bijection.

(2) The map $\pi_{0}\left(L^{\prime}\right) \rightarrow \pi_{0}(S)$ (induced from the inclusion $L^{\prime} \cong S \cap\left(\mathbb{R}^{3} \times\{1\}\right) \hookrightarrow S$ ) is a surjection.

One way to construct such a cobordism is illustrated in Figure 5.2. We may perform a crossing change by a single saddle which adds a meridional circle to one of the strands, and the saddle splits a link component into two. After suitable such crossing changes, we can produce an unlink, with each component having some number meridional circles attached to them. After performing a few more splits using saddles, we can convert this picture into a disjoint union of Hopf links and an unlink. During this cobordism, the only elementary moves that we used were link isotopy and saddles that were splits; therefore, this link cobordism satisfies the above two properties.

Using the maps from Propositions 4.6-4.8 and Definitions 5.1-5.2, we get a map $\mathcal{C}_{\text {fHtot }}(L) \rightarrow$ $\mathcal{C}_{\text {fHtot }}\left(L^{\prime}\right)$ in $\mathcal{K}\left(\mathbb{F}_{2}[W]\right)$. Being a map over $\mathbb{F}_{2}[W]$, the filtrations given by the powers of $W$ are preserved. Therefore, we get a map between the two spectral sequences, see for example, [McC00, Theorem 3.5].

Proposition 5.3 implies the map on the first page is the standard map on the filtered Bar-Natan theory (tensored with $\mathbb{F}_{2}[W]$ ). Since our link cobordism $S$ satisfies the above conditions, for any orientation $o$ on $L$, there exists a unique orientation $o^{\prime}$ on $L^{\prime}$ so that $o$ and $o^{\prime}$ can be extended to an orientation on $S$. Therefore, $(\mathrm{BN}-2)$ implies that the map on $H_{*}\left(\mathcal{C}_{f B N}\right)$ is injective.

Summarizing, we get a map from the spectral sequence for $L$ to the spectral sequences for $L^{\prime}$. It is injective on the homology of the first page; and the spectral sequence for $L^{\prime}$ has no higher differentials. Therefore, the spectral sequence for $L$ has no higher differentials either. (The last step is merely the observation that if $f$ is an injective chain map from a chain complex $\left(C_{1}, d_{1}\right)$ to a chain complex $\left(C_{2}, d_{2}=0\right)$, then $d_{1}=0$.)

Proof of Proposition 5.4. First note that the statements (R-1) and (R-2) are equivalent, since we have an $\mathbb{F}_{2}\left[H, H^{-1}, W\right]$-module gr $h^{\text {-graded isomorphism }}$

$$
\mathcal{C}_{\text {fHtot }} \otimes \mathbb{F}_{2}[\mathbb{Z}]=\left(\mathcal{C}_{\text {tot }} /\{H=1\}\right) \otimes \mathbb{F}_{2}[\mathbb{Z}] \rightarrow\{H\}^{-1} \mathcal{C}_{\text {tot }}
$$

induced by the map

$$
\left[W^{a}(u, x), b\right] \mapsto H^{b-a+\left(\operatorname{gr}_{q}((u, x))-l\right) / 2} W^{a}(u, x) .
$$

(Here the $\mathbb{F}_{2}\left[H, H^{-1}, W\right]$-module structure on $\mathcal{C}_{\text {fHtot }} \otimes \mathbb{F}_{2}[\mathbb{Z}]$ is given by $H^{a} W^{b}\left[W^{c}(u, x), d\right]=$ $\left[W^{b+c}(u, x), a+b+d\right]$.) The map, by definition, is $\mathbb{F}_{2}\left[H, H^{-1}, W\right]$-equivariant; since $\operatorname{gr}_{h}(H)=0$, the map preserves the $\mathrm{gr}_{h}$-grading; and it has an obvious inverse map induced by

$$
H^{a} W^{b}(u, x) \mapsto\left[W^{b}(u, x), a+b+\left(l-\operatorname{gr}_{q}((u, x))\right) / 2\right]
$$


To see that these maps are chain maps, observe that if $H^{c} W^{d}(v, y)$ appears in $\delta_{\text {tot }}((u, x))$, then $\operatorname{gr}_{q}((v, y))=\operatorname{gr}_{q}((u, x))+2 c+2 d$, and therefore, we have a commuting diagram

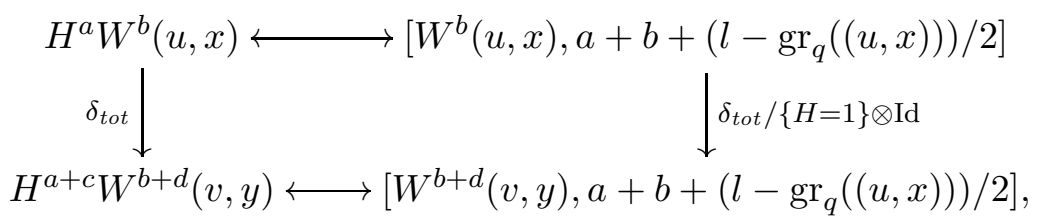

where the horizontal arrows are the maps defined above, and the vertical arrows are parts of the differentials $\delta_{\text {tot }}$ and $\delta_{\text {tot }} /\{H=1\} \otimes \mathrm{Id}$.

The statement (R-3) follows from (R-1) by the following well-known trick in homological algebra (and can also be seen as an application of the universal coefficient theorem). The complex $\mathcal{C}_{\text {ftot }}$ can be viewed as the mapping cone

$$
\mathcal{C}_{\text {ftot }}=\mathcal{C}_{\text {fHtot }} /\{W=1\} \simeq \operatorname{Cone}\left(W-1: \mathcal{C}_{\text {fHtot }} \rightarrow \mathcal{C}_{\text {fHtot }}\right) .
$$

The homology of the mapping cone is the homology of the mapping cone of the homology. That is, we have an exact triangle

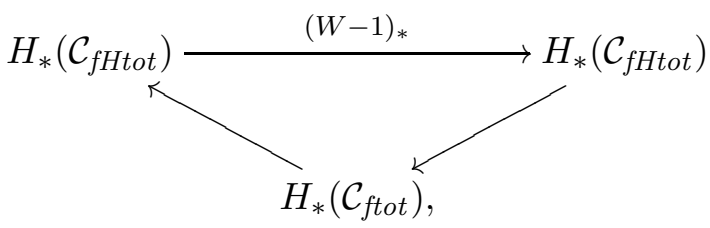

which implies

$$
\begin{aligned}
H_{*}\left(\mathcal{C}_{f t o t}\right) & \cong H_{*}\left(\operatorname{Cone}\left((W-1)_{*}: H_{*}\left(\mathcal{C}_{f H t o t}\right) \rightarrow H_{*}\left(\mathcal{C}_{f H t o t}\right)\right)\right) \\
& =H_{*}\left(\operatorname{Cone}\left(W-1: \bigoplus \mathbb{F}_{2}[W] \rightarrow \bigoplus^{2^{l}} \mathbb{F}_{2}[W]\right)\right) \\
& =\bigoplus \mathbb{F}_{2} .
\end{aligned}
$$

Therefore, we only need to prove the statement for (R-1). This follows immediately from Lemma 5.5. The $E^{2}=E^{\infty}$-page of the spectral sequence $H_{*}\left(\mathcal{C}_{f B N}\right) \otimes \mathbb{F}_{2}[W] \rightrightarrows H_{*}\left(\mathcal{C}_{f H t o t}\right)$ is isomorphic to $2^{l}$ copies of $\mathbb{F}_{2}[W]$, with the copies in a canonical correspondence with the orientations of $L$. The $E^{\infty}$-page being a free module over $\mathbb{F}_{2}[W]$, we do not encounter any extension problems, and can conclude that it is isomorphic to the homology of $\mathcal{C}_{\text {fHtot }}$, which therefore is $2^{l}$ copies of $\mathbb{F}_{2}[W]$ as well.

\section{Concordance invariants}

In this section, we will construct concordance invariants in the same spirit as [Ras10], as described in (BN-3) in Section 5. We concentrate only on knots $K$, although most of the constructions generalize for links. We also only work with the filtered version $\mathcal{C}_{\text {ftot }}(K)$; using the more general 
version would allow us to construct to similar other invariants, although computing them might be more challenging.

Definition 6.1. An upright set is a subset $\mathcal{U}$ of $\mathbb{Z} \times(2 \mathbb{Z}+1)$ satisfying the following condition: If $(a, b)$ is in $\mathcal{U}$ and $a^{\prime} \geq a$ and $b^{\prime} \geq b$, then $\left(a^{\prime}, b^{\prime}\right)$ is also in $\mathcal{U}$. For any even integer $n$, the translate $\mathcal{U}[n]$ is another upright set defined as

$$
(a, b) \in \mathcal{U}[n] \text { if and only if }(a, b-n) \in \mathcal{U} .
$$

A centered upright set is an upright set that contains $(0,1)$, but not $(0,-1)$.

Example 6.1. The intersection of the all the centered upright sets is the following centered upright set

$$
\mathcal{U}_{\text {min }}:=\{(a, b) \mid a \geq 0 \text { and } b>0\},
$$

and the union of the all centered upright sets is the following centered upright set

$$
\mathcal{U}_{\max }:=\{(a, b) \mid a>0 \text { or } b>0\} .
$$

Definition 6.2. A sequence of upright sets $\mathcal{U}_{1}, \mathcal{U}_{2}, \ldots$ is said to have a limit if for all points $(a, b)$, there exists $N$ (depending on $a, b$ ) such that either

(1) $(a, b) \in \mathcal{U}_{i}$ for all $i>N$; or

(2) $(a, b) \notin \mathcal{U}_{i}$ for all $i>N$.

In that case, the limit upright set is defined as

$\lim _{i \rightarrow \infty} \mathcal{U}_{i}=\left\{(a, b) \mid\right.$ there exists $N$ (depending on $a, b$ ) such that for all $\left.i>N,(a, b) \in \mathcal{U}_{i}\right\}$.

The following properties are immediate from the definition.

(1) If a sequence $\mathcal{U}_{1}, \mathcal{U}_{2}, \ldots$ has a limit, then any subsequence also has the same limit.

(2) The limit of centered upright sets, if exists, is centered.

(3) For nested sequences $\mathcal{U}_{1} \subseteq \mathcal{U}_{2} \subseteq, \cdots$, the limit is the union. For nested sequences $\mathcal{U}_{1} \supseteq$ $\mathcal{U}_{2} \supseteq, \cdots$, the limit is the intersection.

Example 6.2. For $t \in[0,1]$, define $\mathcal{U}_{(t)}$ to be the following centered upright set,

$$
\mathcal{U}_{(t)}=\{(a, b) \mid a t+b(1-t)>0 \text { or }[a t+b(1-t)=0 \text { and } b>0]\} .
$$

For any increasing sequence $t_{1} \leq t_{2} \leq \cdots$ of points in $[0,1]$ converging to $t$,

$$
\mathcal{U}_{(t)}=\lim _{i \rightarrow \infty} \mathcal{U}_{\left(t_{i}\right)}
$$

Example 6.3. Extending Example 6.2, for $t \in[0,1], s \in[-1,1]$, consider any function

$$
r:\{(a, b) \mid a t+b(1-t)=s(1-t)\} \rightarrow\{ \pm 1\}
$$

satisfying:

(1) if $a=0, r(0, b)=\operatorname{sgn}(b)$ (only relevant when $t=1$ or $s= \pm 1$ );

(2) if $b$ is fixed, $r(a, b)$ is a non-decreasing function of $a$ (only relevant when $t=0$ ).

Define $\mathcal{U}_{(t, s, r)}$ to be the following centered upright set,

$$
\mathcal{U}_{(t, s, r)}=\{(a, b) \mid a t+b(1-t)>s(1-t) \text { or }[a t+b(1-t)=s(1-t) \text { and } r(a, b)>0]\},
$$

see also Figure 6.1 


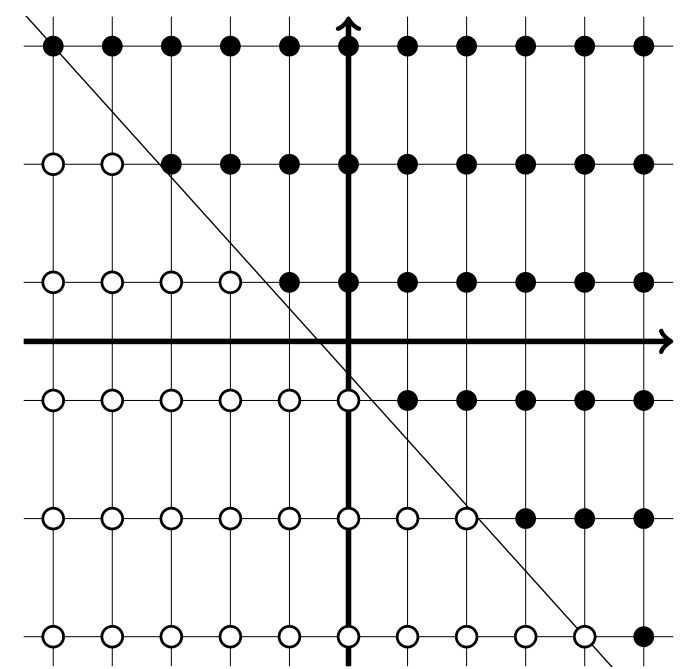

Figure 6.1. The upright set $\mathcal{U}_{(t, s, r)}$ from Example 6.3. Let $\mathscr{L}$ be the line passing through the point $(0, s)$ and making an angle $\theta=2 \tan ^{-1}(t)$ with the negative $x$ axis. The subset $\mathcal{U}_{(t, s, r)} \subset \mathbb{Z} \times(2 \mathbb{Z}+1)$ consists of all the points above and to the right of $\mathscr{L}$ (marked solid), none of the points below and to the left of $\mathscr{L}$ (marked hollow), and some of the points on $\mathscr{L}$ (marked solid or hollow) determined by the function $r$. (If $t=0,1$ or $s= \pm 1$, there are some restrictions on the function $r$ to ensure that $\mathcal{U}_{(t, s, r)}$ is indeed a centered upright set.) We have depicted the case when $t=10 / 19, s=-5 / 9$ and $r$ satisfies $r(-5,5)=1, r(4,-5)=-1$.

Definition 6.3. For any knot $K$, and any upright set $\mathcal{U}$, let $\mathcal{F}_{\mathcal{U}} \mathcal{C}_{f t o t}(K)$ denote the subcomplex of $\mathcal{C}_{f t o t}(K)=(\mathcal{C}(K), \mathbf{d}+\mathbf{h})$ generated by Khovanov generators whose $\left(\operatorname{gr}_{h}, \operatorname{gr}_{q}\right)$-bigradings are in $\mathcal{U}$.

Definition 6.4. Fix a knot $K$ and an orientation $o$ on $K$. For any centered upright set $\mathcal{U}$, define the following three numbers:

$$
\begin{aligned}
s_{o}^{\mathcal{U}}(K) & =\max \left\{n \in 2 \mathbb{Z} \mid \mathcal{F}_{\mathcal{U}[n]} \mathcal{C}_{f t o t}(K) \text { contains a representative for } g(o)\right\}+2 \\
s_{-o}^{\mathcal{U}}(K) & =\max \left\{n \in 2 \mathbb{Z} \mid \mathcal{F}_{\mathcal{U}[n]} \mathcal{C}_{f t o t}(K) \text { contains a representative for } g(-o)\right\}+2 \\
s_{o,-o}^{\mathcal{U}}(K) & =\max \left\{n \in 2 \mathbb{Z} \mid \mathcal{F}_{\mathcal{U}[n]} \mathcal{C}_{f t o t}(K) \text { contains a representative for } g(o)+g(-o)\right\},
\end{aligned}
$$

where $g( \pm o)$ are the two generators for $H_{*}\left(\mathcal{C}_{f t o t}(K)\right)$ corresponding to the orientations $\pm o$, from Proposition 5.4 (R-3).

Example 6.4. Let us compute these new $s$-invariants for the three-crossing diagram of the positive trefoil from Example 3.1. Continuing the same notation from that example, and for some orientation $o$ of the trefoil, the three non-zero elements of the two-dimensional homology of $\mathcal{C}_{\text {ftot }}=$ 
$\mathcal{C}_{\text {tot }} /\{H=W=1\}$ has the following cycle representatives:

$$
\begin{aligned}
g(o) & =\left[x_{1}^{000}+x_{1}^{000} x_{2}^{000}\right] \\
g(-o) & =\left[x_{2}^{000}+x_{1}^{000} x_{2}^{000}\right] \\
g(o)+g(-o) & =\left[x_{1}^{000}+x_{2}^{000}\right] .
\end{aligned}
$$

Since none of $x_{1}^{000}, x_{2}^{000}$, and $x_{1}^{000} x_{2}^{000}$ are hit by the differential, any cycle representative for these elements must contain these Khovanov generators. The $\left(\mathrm{gr}_{h}, \mathrm{gr}_{q}\right)$-bigradings of $x_{1}^{000}, x_{2}^{000}$ and $x_{1}^{000} x_{2}^{000}$ are $(0,3),(0,3)$, and $(0,1)$, respectively. Therefore, for any centered upright set $\mathcal{U}$,

$$
\begin{aligned}
& \mathcal{F}_{\mathcal{U}[n]} \mathcal{C}_{f t o t} \text { contains a cycle representative of } g(o) \text { or } g(-o) \\
\Longleftrightarrow & (0,1) \in \mathcal{U}[n] \\
\Longleftrightarrow & n \leq 0
\end{aligned}
$$

and

$$
\begin{aligned}
& \mathcal{F}_{\mathcal{U}[n]} \mathcal{C}_{f t o t} \text { contains a cycle representative of } g(o)+g(-o) \\
\Longleftrightarrow & (0,3) \in \mathcal{U}[n] \\
\Longleftrightarrow & n \leq 2 .
\end{aligned}
$$

(Here, the last step is justified since the centered upright $\mathcal{U} \subset \mathbb{Z} \times(2 \mathbb{Z}+1)$ contains $(0,1)$ but not $(0,-1)$.) Therefore, $s_{o}^{\mathcal{U}}=s_{-o}^{\mathcal{U}}=s_{o,-o}^{\mathcal{U}}=2$.

It is perhaps not immediate why these numbers are knot invariants. We will prove this in Proposition $6.6(\mathrm{~S}-1)$. Along the way, we need the following lemma.

Lemma 6.5. Consider any connected oriented cobordism from a knot $K_{1}$ to a knot $K_{2}$ in $\mathbb{R}^{3} \times[0,1]$. After viewing the cobordism as a sequence of Reidemeister moves, births, deaths, and saddles, consider the map $f: H_{*}\left(\mathcal{C}_{\text {ftot }}\left(K_{1}\right)\right) \rightarrow H_{*}\left(\mathcal{C}_{f t o t}\left(K_{2}\right)\right)$ induced from the maps defined in Propositions 4.64.8 and Definitions 5.1-5.2. If $\pm o_{1}$ are the orientations on $K_{1}$, and $\pm o_{2}$ are the two corresponding orientations on $K_{2}$ (induced from the connected oriented cobordism), the map acts as follows on the generators of $H_{*}\left(\mathcal{C}_{f t o t}\right)$ :

$$
g\left(o_{1}\right) \mapsto g\left(o_{2}\right) \quad g\left(-o_{1}\right) \mapsto g\left(-o_{2}\right) .
$$

Proof. Consider the induced map $\tilde{f}$ on the partially filtered complex $\mathcal{C}_{\text {fHtot }}=\mathcal{C}_{\text {tot }} /\{H=1\}$. Let $\widetilde{g}\left( \pm o_{1}\right)$ and $\widetilde{g}\left( \pm o_{2}\right)$ be the generators of $H_{*}\left(\mathcal{C}_{f H t o t}\left(K_{1}\right)\right)$ and $H_{*}\left(\mathcal{C}_{f H t o t}\left(K_{2}\right)\right)$ over $\mathbb{F}_{2}[W]$ corresponding to the orientations $\pm o_{1}$ and $\pm o_{2}$, respectively, cf. Proposition 5.4 (R-1). Assume

$$
\begin{aligned}
\widetilde{f}\left(\widetilde{g}\left(o_{1}\right)\right) & =\alpha W^{a} \widetilde{g}\left(o_{2}\right)+\beta W^{b} \widetilde{g}\left(-o_{2}\right) \\
\widetilde{f}\left(\widetilde{g}\left(-o_{1}\right)\right) & =\gamma W^{c} \widetilde{g}\left(o_{2}\right)+\delta W^{d} \widetilde{g}\left(-o_{2}\right)
\end{aligned}
$$

for some $\alpha, \beta, \gamma, \delta \in \mathbb{F}_{2}$, and some integers $a, b, c, d \geq 0$. Here, and elsewhere, we may be viewing the equations at the level of homology, or at the chain level, where $\widetilde{g}\left( \pm o_{i}\right)$ should read 'a cycle representative for $\widetilde{g}\left( \pm o_{i}\right)$ ', and the equality sign should read 'equal relative boundary'. Since $\widetilde{f}$ preserves the $\mathrm{gr}_{h}$-grading, and all of the four elements $\widetilde{g}\left( \pm o_{i}\right)$ live in homological grading zero (Proposition 5.4), we must have $a=b=c=d=0$. 
Therefore, the induced map $f_{B N}$ on the filtered Bar-Natan complex $\mathcal{C}_{f B N}=\mathcal{C}_{\text {tot }} /\{H=1, W=$ $0\}$ is

$$
\begin{aligned}
f_{B N}\left(g_{B N}\left(o_{1}\right)\right) & =\alpha g_{B N}\left(o_{2}\right)+\beta g_{B N}\left(-o_{2}\right) \\
f_{B N}\left(g_{B N}\left(-o_{1}\right)\right) & =\gamma g_{B N}\left(o_{2}\right)+\delta g_{B N}\left(-o_{2}\right)
\end{aligned}
$$

where $g_{B N}\left( \pm o_{i}\right)$ denotes the standard generators of $H_{*}\left(\mathcal{C}_{f B N}\left(K_{i}\right)\right)$ from (BN-1). However, since this induced map $f_{B N}$ is the standard Bar-Natan cobordism map (from Proposition 5.3), (BN-2) implies that $\alpha=\delta=1$ and $\beta=\gamma=0$. Therefore, the map $f$ on $\mathcal{C}_{f t o t}=\mathcal{C}_{\text {tot }} /\{H=W=1\}$ is

$$
g\left(o_{1}\right) \mapsto g\left(o_{2}\right) \quad g\left(-o_{1}\right) \mapsto g\left(-o_{2}\right) .
$$

as desired.

Proposition 6.6. These new s-invariants for any knot $K$ and any centered upright set $\mathcal{U}$, satisfy the following.

$(S-1)$ Each of the three numbers $s_{*}^{\mathcal{U}}(K)$ is a knot invariant, and each is zero for the unknot.

$\left(S\right.$-2) If $\mathcal{U}^{\prime}$ is another centered upright set with $\mathcal{U} \subset \mathcal{U}^{\prime}$, then for each of the three variants

$$
s_{*}^{\mathcal{U}}(K) \leq s_{*}^{\mathcal{U}^{\prime}}(K) .
$$

(S-3) For any sequence of centered upright sets $\mathcal{U}_{1}, \mathcal{U}_{2}, \ldots$ that have a limit, each of the three variants satisfy

$$
\lim _{i \rightarrow \infty} s_{*}^{\mathcal{U}_{i}}(K)=s_{*}^{\lim _{i} \mathcal{U}_{i}}(K) .
$$

$(S-4) s_{o,-o}^{\mathcal{U}}+2 \geq s_{o}^{\mathcal{U}}(K)=s_{-o}^{\mathcal{U}}(K)$.

$(S-5)$ For each variant, $s_{*}^{\mathcal{U}_{(1)}}(K)$ agrees with the Rasmussen s-invariant (where $\mathcal{U}_{(1)}$ is described in Example 6.2).

(S-6) Each of the three numbers $s_{*}^{\mathcal{U}}(K)$ is finite.

Proof. For the proof of (S-1), note that the bigraded chain homotopy type of the full theory $\mathcal{C}_{\text {tot }}(K)$ is a knot invariant, see Proposition 4.2; therefore, the $\left(\mathrm{gr}_{h}, \mathrm{gr}_{q}\right)$-bifiltered chain homotopy type of $\mathcal{C}_{f t o t}(K)$ is a knot invariant as well. Furthermore, the maps inducing the homotopy equivalence preserve the generators corresponding to $\pm o$, see Lemma 6.5 ; therefore, each of the three numbers is a knot invariant. The computation for the unknot is immediate from the 0-crossing diagram of the unknot.

For (S-2), observe that for all $n, \mathcal{F}_{\mathcal{U}[n]} \mathcal{C}_{f t o t}(K)$ is a subcomplex of $\mathcal{F}_{\mathcal{U}^{\prime}[n]} \mathcal{C}_{f t o t}(K)$. Consequently, if $\mathcal{F}_{\mathcal{U}[n]} \mathcal{C}_{f t o t}(K)$ contains a cycle representative for some element in $H_{*}\left(\mathcal{C}_{\text {ftot }}(K)\right)$, so does $\mathcal{F}_{\mathcal{U}^{\prime}[n]} \mathcal{C}_{\text {ftot }}(K)$.

For (S-3), fix some knot diagram for $K$. The Khovanov chain group $\mathcal{C}$ for this knot diagram is supported on some finite subset of $\mathbb{Z} \times(2 \mathbb{Z}+1)$. Choose $N$ large enough so that for all $i>N, \mathcal{U}_{i}$ agrees with $\lim _{i} \mathcal{U}_{i}$ on this finite subset. The claim then follows immediately.

For the equality in (S-4), we will produce an automorphism of $\mathcal{C}_{\text {tot }}(K)$ (in $\mathcal{K}\left(\mathbb{F}_{2}[H, W]\right)$ ) whose induced automorphism on $H_{*}\left(\mathcal{C}_{f t o t}(K)\right)$ interchanges $g(o)$ and $g(-o)$. Fix some knot diagram $D_{1}$ for $K$, and consider the rightmost strand. We may move it over the point at $\infty$ in $S^{2}$ to obtain a diagram $D_{2}$. This isotopy in $S^{2}$ induces an identification between $\mathcal{C}_{\text {tot }}\left(D_{1}\right)$ and $\mathcal{C}_{\text {tot }}\left(D_{2}\right)$. We then perform a sequence of Reidemeister moves in the plane $\mathbb{R}^{2}$ to get back to $D_{1}$ from $D_{2}$, producing 


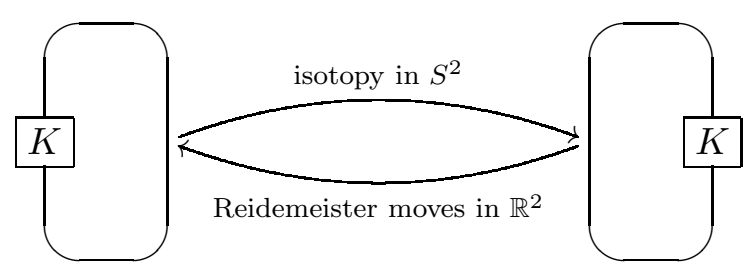

FiguRE 6.2. An automorphism of $\mathcal{C}_{\text {tot }}$ that reverses the orientation.

a map $\mathcal{C}_{\text {tot }}\left(D_{2}\right) \rightarrow \mathcal{C}_{\text {tot }}\left(D_{1}\right)$. The composition is the required automorphism. See Figure 6.2 (the same trick was used in [Kho03] to deal with the basepoint). Since the isotopy moves over $\infty \in S^{2}$ once, the checkerboard coloring in $(\mathrm{BN}-1)$ is reversed, and therefore, the induced automorphism of the filtered Bar-Natan complex $\mathcal{C}_{f B N}=\mathcal{C}_{f H t o t} /\{W=0\}$ interchanges the two generators. As in the proof of Lemma 6.5, this shows that the automorphism on $\mathcal{C}_{\text {ftot }}$ interchanges the two generators as well.

The inequality in (S-4) follows immediately from the observation that if $\mathcal{F}_{\mathcal{U}[n]} \mathcal{C}_{f t o t}(K)$ contains cycle representatives for $g(o)$ and $g(-o)$, then it contains a cycle representative for $g(o)+g(-o)$ as well.

The proof of (S-5) takes up most of the work. Let $s$ denote the Rasmussen invariant. Assume we are working with the variant $s_{o}^{\mathcal{U}_{(1)}}$ (the argument for the other variants are similar). To show $s_{o}^{\mathcal{U}_{(1)}} \geq s$, we need to show $\mathcal{F}_{\mathcal{U}_{(1)}[s-2]} \mathcal{C}_{\text {ftot }}$ contains a cycle representative for $g(o)$; and to show $s_{o}^{\mathcal{U}_{(1)}} \leq s$, we need to show $\mathcal{F}_{\mathcal{U}_{(1)}[s]} \mathcal{C}_{\text {ftot }}$ does not contain a representative for $g(o)$.

For convenience, let us fix a few more notations. Let $\mathbf{f}=\mathbf{d}_{1}+\mathbf{h}_{1}$ and $\mathbf{g}=\mathbf{d}+\mathbf{h}-\mathbf{f}$. Both are endomorphisms on the total chain group $\mathcal{C}$; neither drops the quantum grading $\operatorname{gr}_{q}, \mathbf{f}$ increases $\operatorname{gr}_{h}$ by one, while $\mathbf{g}$ increases it by at least two. Furthermore, we have $\mathbf{f}^{2}=[\mathbf{f}: \mathbf{g}]=\mathbf{g}^{2}=0$. Also, let $\mathcal{C}^{\geq i}$ be the subgroup of $\mathcal{C}$ that lives in homological grading at least $i$, and $\mathcal{C}^{i}$ be the subgroup of $\mathcal{C}$ that lives in homological grading $i$.

Recall from $(\mathrm{BN}-1)$, the $\mathrm{gr}_{h}$-graded chain complex $\mathcal{C}_{f B N}=(\mathcal{C}, \mathbf{f})$ has homology of rank two, generated by $g_{B N}(o)$ and $g_{B N}(-o)$, both supported in $\mathrm{gr}_{h}$-grading zero. Using (BN-3), choose a cycle representative $c_{0}$ for $g_{B N}(o)$ living in $\mathcal{C}^{0} \cap \mathcal{F}_{\mathcal{U}_{(1)}[s-2]} \mathcal{C}$. Therefore, we have $\mathbf{f}\left(c_{0}\right)=0$; let $b_{0}=\mathbf{g}\left(c_{0}\right) \in \mathcal{C}^{\geq 2}$. Now assume by induction that we have defined, for $i=0, \ldots, k-1$, chains $b_{i} \in \mathcal{C}^{\geq i+2}$ and $c_{i} \in \mathcal{C}^{\geq i}$, so that $\mathbf{g}\left(c_{i}\right)=b_{i}$, and (for $\left.i \neq 0\right) \mathbf{f}\left(c_{i}\right)=b_{i-1}$. We will extend the construction to $i=k$. Since

$$
\mathbf{f}\left(b_{k-1}\right)=\mathbf{f g}\left(c_{k-1}\right)=\mathbf{g} \mathbf{f}\left(c_{k-1}\right)= \begin{cases}0 & \text { if } k=1 \\ \mathbf{g}\left(b_{k-2}\right)=\mathbf{g}^{2}\left(c_{k-2}\right)=0 & \text { if } k>1\end{cases}
$$

and $b_{k-1}$ lives in homological grading at least $k+1$, and the entire homology of $(\mathcal{C}, \mathbf{f})$ is supported in grading zero, there is some chain $c_{k} \in \mathcal{C}^{\geq k}$ with $\mathbf{f}\left(c_{k}\right)=b_{k-1}$; define $b_{k}=\mathbf{g}\left(c_{k}\right)$. Then $\sum_{i} c_{i}$ is a cycle for the chain complex $\mathcal{C}_{f t o t}=(\mathcal{C}, \mathbf{f}+\mathbf{g})$, and indeed, represents the generator $g(o)$. Moreover, by construction, it is supported in $\mathcal{F}_{\mathcal{U}_{(1)}[s-2]} \mathcal{C}$, and this establishes $s_{o} \mathcal{U}_{(1)} \geq s$. 
For the other direction, assume if possible, $g(o)$ has a cycle representative in $\mathcal{F}_{\mathcal{U}_{(1)}[s]} \mathcal{C}$. Let $c_{i}^{\prime}$ be the part of this cycle representative that lives in homological grading $i$. Therefore, $\sum_{i}\left(c_{i}+c_{i}^{\prime}\right)$ is a boundary, say $(\mathbf{f}+\mathbf{g})(a)$, for some chain $a$; let $a_{i}$ be the part of $a$ that lives in $\operatorname{gr}_{h}=i$, and let $k=\min \left\{i \mid a_{i} \neq 0\right\}$. Since $\left(c_{0}+c_{0}^{\prime}\right) \neq 0$, we have $k \leq-1$. Indeed, we may assume $k=-1$. Otherwise, if $k<-1$, then $\mathbf{f}\left(a_{k}\right) \in \mathcal{C}^{k+1}$ is zero, and since the homology of $(\mathcal{C}, \mathbf{f})$ is supported in grading zero, there exists a chain $e \in \mathcal{C}^{k-1}$ with $\mathbf{f}(e)=a_{k}$. Then $(a+(\mathbf{f}+\mathbf{g})(e)) \in \mathcal{C} \geq k+1$ is another chain whose boundary is also $\sum_{i}\left(c_{i}+c_{i}^{\prime}\right)$. Therefore, we may assume, $a \in \mathcal{C}^{\geq-1}$. Then we must have $\mathbf{f}\left(a_{-1}\right)=c_{0}+c_{0}^{\prime}$. This implies $c_{0}^{\prime}$ is also a cycle representative for the generator $g_{B N}(o)$ in $H_{*}\left(\mathcal{C}_{f B N}\right)$. Since $c_{0}^{\prime}$ is supported in quantum grading $\operatorname{gr}_{q} \geq s+1$, this is a contradiction, thereby establishing $s_{o}^{\mathcal{U}_{(1)}} \leq s$.

The statement of (S-6) is an immediate corollary of (S-5). Let us assume that we are working with the variant $s_{o}^{\mathcal{U}}(K)$ (the argument for the other variants are similar). Since

$$
s_{o}^{\mathcal{U}_{\min }}(K) \leq s_{o}^{\mathcal{U}}(K) \leq s_{o}^{\mathcal{U}_{\max }}(K),
$$

from (S-2) (where $\mathcal{U}_{\text {min }}$ and $\mathcal{U}_{\text {max }}$ are defined in Example 6.1), it is enough to show that $s_{o}^{\mathcal{U}_{\min }}(K)>$ $-\infty$ and $s_{o}^{\mathcal{U}_{\max }}(K)<\infty$. Let $s=s_{o}^{\mathcal{U}_{(1)}}(K)$ be Rasmussen's $s$-invariant. Then from definitions, $\mathcal{F}_{\mathcal{U}_{(1)}[s-2]} \mathcal{C}_{\text {ftot }}(K)$ contains a representative for $g(o)$, but $\mathcal{F}_{\mathcal{U}_{(1)}[s]} \mathcal{C}_{\text {ftot }}(K)$ does not.

Now fix some knot diagram for $K$, and let $S$ be the finite subset of $\mathbb{Z} \times(2 \mathbb{Z}+1)$ that supports the bigrading of the Khovanov chain complex $\mathcal{C}$ for this knot diagram. There exists $n$ sufficiently small so that

$$
\mathcal{U}_{(1)}[s-2] \cap S \subset \mathcal{U}_{\min }[n]
$$

and therefore, $\mathcal{F}_{\mathcal{U}_{\text {min }}[n]} \mathcal{C}_{f t o t}(K)$ contains a cycle representative for $g(o)$ as well. Similarly, there exists $m$ sufficiently large so that

$$
\mathcal{U}_{\max }[m] \cap S \subset \mathcal{U}_{(1)}[s],
$$

and therefore, $\mathcal{F}_{\mathcal{U}_{\max }[m]} \mathcal{C}_{f t o t}(K)$ cannot contain a cycle representative for $g(o)$.

We conclude by observing that each of these new $s$-invariants produce a lower bound for the four-ball genus.

Proposition 6.7. For any connected oriented genus-g knot cobordism between any knots $K_{1}$ and $K_{2}$ in $\mathbb{R}^{3} \times[0,1]$, and any centered upright set $\mathcal{U}$,

$$
g \geq \frac{1}{2}\left|s_{*}^{\mathcal{U}}\left(K_{1}\right)-s_{*}^{\mathcal{U}}\left(K_{2}\right)\right| .
$$

Therefore, the four-ball genus of any knot $K$ is bounded below

$$
g_{4}(K) \geq \frac{1}{2}\left|s_{*}^{\mathcal{U}}(K)\right|
$$

(Here $s_{*}^{\mathcal{U}}$ denotes any one of the three versions defined in Definition 6.4.)

Proof. Present the cobordism as a sequence of elementary moves, and consider the link-cobordism map $f: \mathcal{C}_{\text {tot }}\left(K_{1}\right) \rightarrow \mathcal{C}_{\text {tot }}\left(K_{2}\right)\{-2 g\}$ in $\mathcal{K}\left(\mathbb{F}_{2}[H, W]\right)$ as defined in Propositions 4.6-4.8 and Definitions 5.1-5.2. (This map might depend on how the cobordism is presented, but that turns out to be irrelevant.) We will analyze the map $\bar{f}$ induced on $\mathcal{C}_{\text {ftot }}=\mathcal{C}_{\text {tot }} /\{H=W=1\}$. 
Let $(u, x)$ and $(v, y)$ be Khovanov generators so that $(v, y)$ appears in $\bar{f}((u, x))$. Then $(v, y)$ must appear in $f((u, x))$ with some non-zero coefficient, say $H^{a} W^{b}$, for some $a, b \geq 0$. Therefore,

$$
\begin{aligned}
& \operatorname{gr}_{h}((v, y))=b+\operatorname{gr}_{h}\left(\left(H^{a} W^{b}(v, y)\right)=b+\operatorname{gr}_{h}((u, x)) \geq \operatorname{gr}_{h}((u, x)),\right. \text { and } \\
& \operatorname{gr}_{q}((v, y))=2(a+b)+\operatorname{gr}_{q}\left(\left(H^{a} W^{b}(v, y)\right)=2(a+b)+\operatorname{gr}_{q}((u, x))-2 g \geq \operatorname{gr}_{q}((u, x))-2 g .\right.
\end{aligned}
$$

In other words, if $(u, x)$ is contained in any upright set $\mathcal{U}^{\prime}$, then $(v, y)$ is contained in the translate $\mathcal{U}^{\prime}[-2 g]$.

From Lemma 6.5, we know that

$$
\bar{f}\left(g\left(o_{1}\right)\right)=g\left(o_{2}\right) \quad \bar{f}\left(g\left(-o_{1}\right)\right)=g\left(-o_{2}\right),
$$

where $\pm o_{1}$ and $\pm o_{2}$ are the orientations on $K_{1}$ and $K_{2}$, induced from the two orientations of the cobordism.

Summarizing what we have said so far, for every $n \in \mathbb{Z}$, we have a commuting diagram

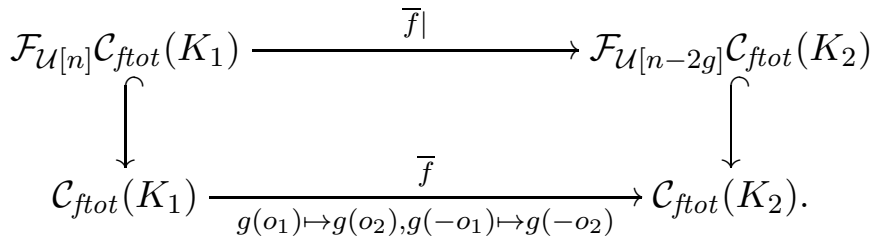

Therefore, if $\mathcal{F}_{\mathcal{U}[n]} \mathcal{C}_{f t o t}\left(K_{1}\right)$ contains a cycle representative for $g\left(o_{1}\right)$ (respectively, $g\left(o_{1}\right)+g\left(-o_{1}\right)$ ), then $\mathcal{F}_{\mathcal{U}[n-2 g]} \mathcal{C}_{\text {ftot }}\left(K_{2}\right)$ contains a cycle representative for $g\left(o_{2}\right)$ (respectively, $g\left(o_{2}\right)+g\left(-o_{2}\right)$ ). Therefore, we get the inequality $s_{*}^{\mathcal{U}}\left(K_{2}\right) \geq s_{*}^{\mathcal{U}}\left(K_{1}\right)-2 g$.

Viewing the cobordism in reverse, we get $s_{*}^{\mathcal{U}}\left(K_{1}\right) \geq s_{*}^{\mathcal{U}}\left(K_{2}\right)-2 g$, and combining the two inequalities, we reach our desired goal.

One can easily construct model chain complexes where these new $s$-invariants are different from Rasmussen's $s$-invariant. The following are perhaps the simplest of such models (the two models are duals of one another). Assume that $(\mathcal{C}, \mathbf{d}+\mathbf{h})$ contains a direct summand in one of the following two forms, and assume $b$ is a representative for the Bar-Natan generator $g_{B N}(o)$. Therefore, $b$ lies in bigrading $(0, s-1)$, where $s$ is the Rasmussen invariant.
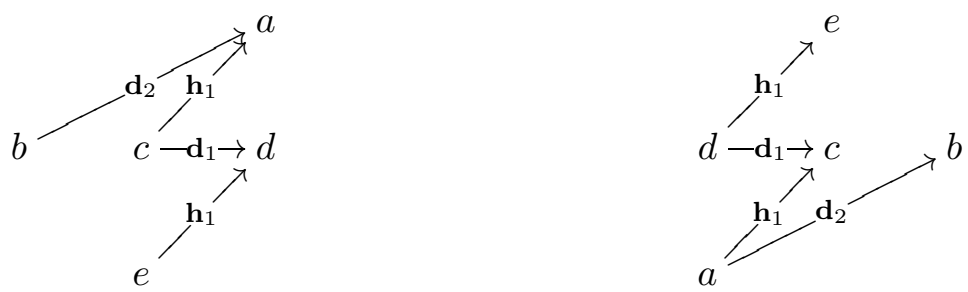

In the first case, we get that $g(o)$ has a unique cycle representative $b+c+e$, and therefore, for any centered upright set $\mathcal{U}$,

$$
s_{o}^{\mathcal{U}}= \begin{cases}s & \text { if }(1,-1) \in \mathcal{U}, \\ s-2 & \text { otherwise }\end{cases}
$$


In the second case, $g(o)$ has three cycle representatives, $b, c$, or $e$; and therefore, for any centered upright set $\mathcal{U}$,

$$
s_{o}^{\mathcal{U}}= \begin{cases}s+2 & \text { if }(-1,3) \in \mathcal{U}, \\ s & \text { otherwise. }\end{cases}
$$

Note that in both cases, the $E^{3}$-page of the Bar-Natan spectral sequence, induced from the filtered Bar-Natan complex $\mathcal{C}_{f B N}=\left(\mathcal{C}, \mathbf{d}_{1}+\mathbf{h}_{1}\right)$, contains one of the following two configurations (the higher differential is a zigzag differential from Equation (4.1)):
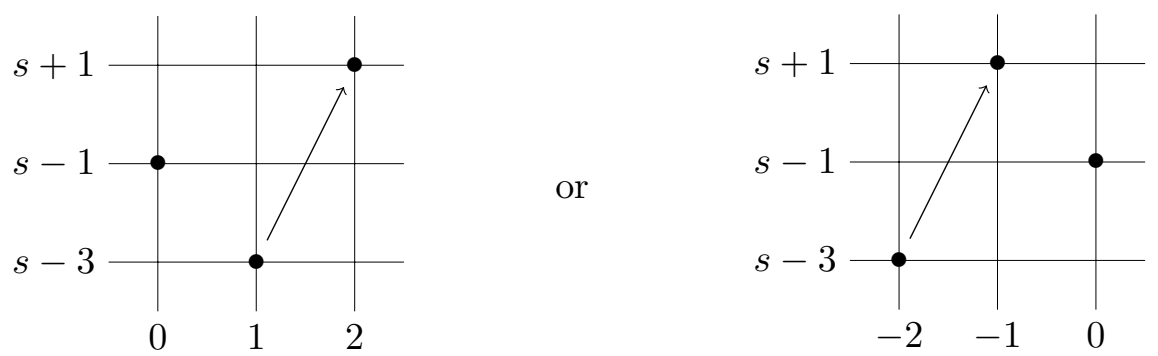

However, the Bar-Natan spectral sequence usually collapses very quickly. Indeed, for all knots up to 16 crossings, except the connect sum of the torus knot $T(3,4)$ with its mirror, the $E^{3}$-page of the Bar-Natan spectral sequence does not contain any of the above two configurations. Therefore, it remains a challenging exercise to find knots where these new $s$-invariants are different from the existing one.

\section{REFERENCES}

[AS] Mohammed Abouzaid and Ivan Smith, Khovanov homology from Floer cohomology, arXiv:1504.01230.

[Blo11] Jonathan M. Bloom, A link surgery spectral sequence in monopole Floer homology, Adv. Math. 226 (2011), no. 4, 3216-3281. MR 2764887

[BN05] Dror Bar-Natan, Khovanov's homology for tangles and cobordisms, Geom. Topol. 9 (2005), $1443-1499$. MR 2174270 (2006g:57017)

[CMW09] David Clark, Scott Morrison, and Kevin Walker, Fixing the functoriality of Khovanov homology, Geom. Topol. 13 (2009), no. 3, 1499-1582. MR 2496052 (2010k:57023)

[Jac04] Magnus Jacobsson, An invariant of link cobordisms from Khovanov homology, Algebr. Geom. Topol. 4 (2004), 1211-1251 (electronic). MR 2113903 (2005k:57047)

[Kho00] Mikhail Khovanov, A categorification of the Jones polynomial, Duke Math. J. 101 (2000), no. 3, 359-426. MR 1740682 (2002j:57025)

[Kho02] _ A functor-valued invariant of tangles, Algebr. Geom. Topol. 2 (2002), 665-741 (electronic). MR 1928174 (2004d:57016)

[Kho03] _ Patterns in knot cohomology. I, Experiment. Math. 12 (2003), no. 3, 365-374. MR 2034399 (2004m:57022)

[Kho06] _ An invariant of tangle cobordisms, Trans. Amer. Math. Soc. 358 (2006), no. 1, 315-327 (electronic). MR 2171235 (2006g:57046)

[KM93] P. B. Kronheimer and T. S. Mrowka, Gauge theory for embedded surfaces. I, Topology 32 (1993), no. 4, 773-826. MR 1241873 (94k:57048)

[KM11] Khovanov homology is an unknot-detector, Publ. Math. Inst. Hautes Études Sci. (2011), no. 113, 97-208. MR 2805599

[KR08a] Mikhail Khovanov and Lev Rozansky, Matrix factorizations and link homology, Fund. Math. 199 (2008), no. 1, 1-91. MR 2391017 (2010a:57011) 
[KR08b] _ Matrix factorizations and link homology. II, Geom. Topol. 12 (2008), no. 3, 1387-1425. MR 2421131 (2010g:57014)

[Lee05] Eun Soo Lee, An endomorphism of the Khovanov invariant, Adv. Math. 197 (2005), no. 2, 554-586. MR 2173845 (2006g:57024)

[LS14] Robert Lipshitz and Sucharit Sarkar, A refinement of Rasmussen's s-invariant, Duke Math. J. 163 (2014), no. 5, 923-952. MR 3189434

[Man06] Ciprian Manolescu, Nilpotent slices, Hilbert schemes, and the Jones polynomial, Duke Math. J. 132 (2006), no. 2, 311-369. MR 2219260 (2007k:53151)

[McC00] John McCleary, A user's guide to spectral sequences, second ed., Cambridge University Press, 2000, Cambridge Books Online.

[Nao06] Gad Naot, The universal Khovanov link homology theory, Algebr. Geom. Topol. 6 (2006), 1863-1892 (electronic). MR 2263052 (2007k:57022)

[Ng05] Lenhard Ng, A Legendrian Thurston-Bennequin bound from Khovanov homology, Algebr. Geom. Topol. 5 (2005), 1637-1653. MR 2186113 (2007g:57027)

[ORSz13] Peter S Ozsváth, Jacob Rasmussen, and Zoltán Szabó, Odd Khovanov homology, Algebr. Geom. Topol. 13 (2013), no. 3, 1465-1488. MR 3071132

[OSz04] Peter Ozsváth and Zoltán Szabó, Holomorphic disks and topological invariants for closed three-manifolds, Ann. of Math. (2) 159 (2004), no. 3, 1027-1158. MR 2113019 (2006b:57016)

[OSz05] On the Heegaard Floer homology of branched double-covers, Adv. Math. 194 (2005), no. 1, 1-33. MR 2141852 (2006e:57041)

[Ras10] Jacob Andrew Rasmussen, Khovanov homology and the slice genus, Invent. Math. 182 (2010), no. 2, 419-447. MR 2729272 (2011k:57020)

[SS06] Paul Seidel and Ivan Smith, A link invariant from the symplectic geometry of nilpotent slices, Duke Math. J. 134 (2006), no. 3, 453-514. MR 2254624 (2007f:53118)

[SS10] L L L L _ _ _ 2010), no. 6, $1464-1501$. MR 2739000 (2012e:53182)

[Szab] Zoltán Szabó, A geometric spectral sequence in Khovanov homology, arXiv:1010.4252.

[Tur06] Paul R. Turner, Calculating Bar-Natan's characteristic two Khovanov homology, J. Knot Theory Ramifications 15 (2006), no. 10, 1335-1356. MR 2286127 (2008b:57014)

E-mail address: sucharit@math.princeton.edu

E-mail address: cseed@math.princeton.edu

E-mail address: szabo@math.princeton.edu

Department of Mathematics, Princeton University, Princeton, NJ 08544 